\title{
An Upscaled Model for Bio-Enhanced NAPL Dissolution in Porous Media
}

\author{
T. Bahar ${ }^{1}$ - F. Golfier ${ }^{1}$ - C. Oltéan ${ }^{1} \cdot$ M. Benioug ${ }^{1}$
}

\begin{abstract}
We develop a Darcy-scale model for multiphase transport in porous media colonized by biofilms. We start with the pore scale description of mass transfer within and between the phases (water, biofilm, and NAPL phases) and biologically mediated reactions. The macroscale mass balance equations under local mass equilibrium condition at the fluidbiofilm interface are derived from the pore scale problem, obtained by the method of volume averaging. The case of local mass equilibrium considered here finally provides one mass balance equation for the fluid and biological phases coupled with the NAPL-phase equation. We predict the effective dispersion tensor and the mass exchange coefficient that appear in the upscaled equation by solving closure problems on representative unit cells. The results of this model have been compared with pore scale simulations. Based on these comparisons, the validity domain of this model has been identified in terms of hydrodynamic and biochemical conditions of transport (i.e., Péclet and Damköhler numbers). This study should provide a better insight on the impact of biofilm dynamics near NAPL sources through the upscaling process.
\end{abstract}

Keywords Porous media · Biofilm · NAPL · Upscaling · Volume averaging

\section{List of Symbols}

$A_{i j} \quad$ Interface between the $i$-phase and $j$-phase $(i, j: \omega, \beta, \gamma, k)$

$\mathbf{b}_{\beta} \quad$ Closure variable that maps $\nabla\left\langle c_{A \beta}\right\rangle^{\beta}$ onto $\tilde{c}_{A \beta}(\mathrm{m})$

$\mathbf{b}_{\omega} \quad$ Closure variable that maps $\nabla\left\langle c_{A \omega}\right\rangle^{\omega}$ onto $\tilde{c}_{A \omega}(\mathrm{m})$

$\mathbf{b}_{\beta}^{\prime} \quad$ Dimensionless form of the closure variable $\mathbf{b}_{\beta}(-)$

$\mathbf{b}_{\omega}^{\prime} \quad$ Dimensionless form of the closure variable $\mathbf{b}_{\omega}(-)$

T. Bahar

tidjani.bahar@univ-lorraine.fr

1 Université de Lorraine, CNRS, CREGU, GeoRessources Laboratory, 54518 Vandœuvre-lès-Nancy Cedex, France 


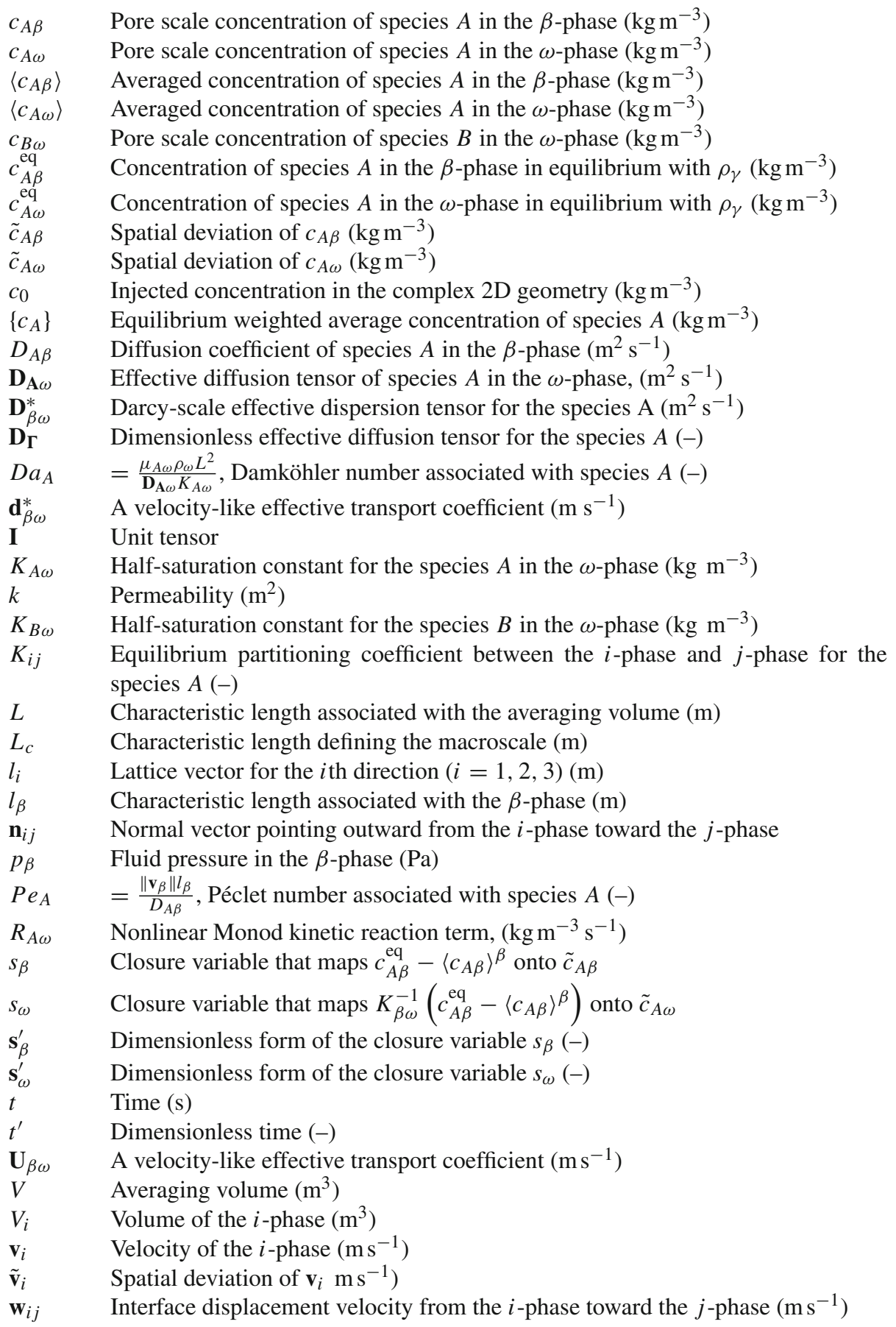




\section{Greek symbols}

$\begin{array}{ll}\alpha_{A}^{*} & \text { Mass transfer coefficient for the species } A\left(\mathrm{~m} \mathrm{~s}^{-1}\right) \\ \beta & \text { Fluid phase } \\ \gamma & \text { NAPL phase } \\ \epsilon_{i} & \text { Volume fraction of the } i \text {-phase } \\ \mu_{\beta} & \text { Fluid dynamic viscosity (Pa.s) } \\ \mu_{A \omega} & \text { Specific degradation rate for the species } A \text { in the } \omega \text {-phase }\left(\mathrm{s}^{-1}\right) \\ \rho_{\omega} & \text { Microbial density in the } \omega \text {-phase }\left(\mathrm{kg} \mathrm{m}^{-3}\right) \\ \rho_{\gamma} & \text { NAPL-phase density }\left(\mathrm{kg} \mathrm{m}^{-3}\right) \\ \rho_{\beta} & \text { Water-phase density }\left(\mathrm{kg} \mathrm{m}^{-3}\right) \\ \sigma & \text { Solid phase } \\ \omega & \text { Biofilm phase } \\ \langle.\rangle^{i} & \text { Intrinsic average for the } i \text {-phase } \\ \langle.\rangle & \text { Superficial average }\end{array}$

\section{Introduction}

Contamination of groundwater by chemical solvent and other industrial waste has become matter of increasing public concern (Quintard and Whitaker 1994; Schubert et al. 2007). In particular, contamination of aquifers by non-aqueous-phase liquids (NAPLs) represents a serious issue for conventional cleanup methods and hence a real challenge for bioremediation techniques. This contamination leads to the formation of NAPL blobs or ganglia trapped in the soil which act as a long-term source of groundwater pollution (Quintard and Whitaker 1994). Transport modeling of non-aqueous-phase liquids (NAPLs) in soils and aquifers has received a lot of attention in the past. Most of the proposed models (Mayer and Miller 1993; Hunt and Sitar 1988; Corapcioglu et al. 2009) consider a two- or three-phase system that lead to explore the NAPL blobs dissolution and mobilization in porous media. The interest in NAPL dissolution enhancement due to biological activity, however, is more recent. In Fig. 1, we illustrate this situation, where $\beta$ refers to the flowing water, NAPL blobs are denoted by $\gamma, \omega$ is the biofilm phase, and $\sigma$ refers to the solid phase.

Modeling NAPL transport involving microbiological processes is complex because of the biological, physical, and chemical processes involved at different scales. A literature survey of the impact of biogeochemical processes on the fate of pollutant plume can be found in Brun and Engesgaard (2002). Bacterial populations, mainly present at the surface of the grains as biofilms (Orgogozo et al. 2013), are found to be the primary attenuation mechanism for organic solute in groundwater. Biofilm has important applications in variety of technologies including bioremediation (Semprini and McCarty 1991; Cirpka et al. 1999) and more recently has been of interest in applications of microbial oil-enhanced recovery where bacteria are utilized for oil mobilization in a reservoir (Sivasankar and Suresh 2014; Soudmand-asli et al. 2007; Armstrong and Wildenschild 2012). In addition, the phenomenon of bacterial chemotaxis increases significantly contaminant degradation in soils and aquifers (Valdès-Parada et al. 2009).

The most of studies (Kindred and Celia 1989; Clement et al. 2004; Golfier et al. 2009; Becker and Seagren 2009; Davit et al. 2010; Chambon et al. 2010; Orgogozo et al. 2013) interested in solute transport in porous media colonized by biofilms are focused away from the pollution source. These models, however, usually fail to predict the fate of pollutant 
plume precisely since the biochemical interactions close to NAPL source are ignored and most of the key parameters need to be fitted or determined a priori (Gaganis et al. 2002; Chu et al. 2007). One method to address this latter issue is to keep an explicit coupling between the microscale information and these macroscopic coefficients through an upscaling process. In Golfier et al. (2009), for instance, a one-equation model has been derived to describe the Darcy-scale transport of a solute undergoing biodegradation in porous media. This model is obtained from the method of volume averaging under local mass equilibrium assumption.

Significant research effort has been put into investigating the transport and biodegradation of dissolved contaminants (Clement et al. 2004; Golfier et al. 2009; Becker and Seagren 2009; Davit et al. 2010; Brun and Hatfield 2011), but comparatively very few works (Christ and Abriola 2006; Chen et al. 2013) are focused on the study of three-phase system (oilwater-biofilm system). Nevertheless, these flow conditions play a key role in the overall process of in situ biodegradation. In a three-phase system with a single component exchanging through the phase interfaces, indeed, mass exchange between phases is classically described by a partitioning coefficient or even a simple Dirichlet condition in a pure binary case. For multispecies systems, the problem is more complex; under certain conditions, ideal solubilization can be considered and mass transfer of dissolved compounds is thus assumed to obey to equilibrium relationships such as Raoult's law. Anyway, in both cases, significant questions remain in the presence of bacterial activity. How does the biofilm affect interfacial mass transfer processes and NAPL solubility? And, conversely, how does the toxicity of high concentration of contaminants near NAPL sources impact the viability and the dynamics of contaminant-degrading bacteria?

Several studies have shown an increase in NAPL dissolution in the presence of microorganisms (Yang and McCarty 2000; Armstrong and Wildenschild 2012). Microorganisms produce amphiphilic compounds, termed biosurfactant, which reduce interfacial tension between immiscible phases (Armstrong and Wildenschild 2012). Paulsen et al. (1999) have obtained images showing the impact of biosurfactants on the oil droplet, where the bacteria were strongly adhered to the oil phase. These images highlight the existence of a local change in interfacial tension that leads to the rupture of the oil surface (Paulsen et al. 1999). In the presence of non-miscible contaminant, additional feedback mechanisms may also appear with the impact of NAPL toxicity effect on biofilm growth (Ray and Peters 2008; Singh and Olson 2009). Ray and Peters (2008) reported inhibition in both anabolism and catabolism of Pseudomonas aeruginosa as a result of exposure to high concentrations of NAPL. Singh and Olson (2009) evaluated the toxic effect of high concentrations of NAPL on bacteria under prolonged exposure, and results from this work indicate that the bacterial cells near the NAPL become non-viable in time, whereas cells accumulating farther away use chemotaxis to migrate toward regions with optimal chemical concentrations in the form of concentrated bacterial bands. This optimal concentration region corresponds to a balance between a minimum level of contaminant toxicity and the highest available nutrient concentration.

In the present paper, we extend the theoretical results obtained by Golfier et al. (2009), Davit et al. (2010), and Orgogozo et al. (2013) to three-phase systems. The research effort will be mainly focused on the impact of the additional phase (NAPL phase) on transport and biodegradation processes rather than the biofilm growth dynamics itself (changes in biofilm distribution in the porous medium for multiphase systems).

The goals of this work were (1) to develop a macroscopic model of multiphase transport at the Darcy scale through volume averaging, based on the data available at pore scale; (2) to calculate the effective properties and determine the effect of pore scale processes (biofilm growth, NAPL blob distribution...) on these effective properties; and (3) to determine the 


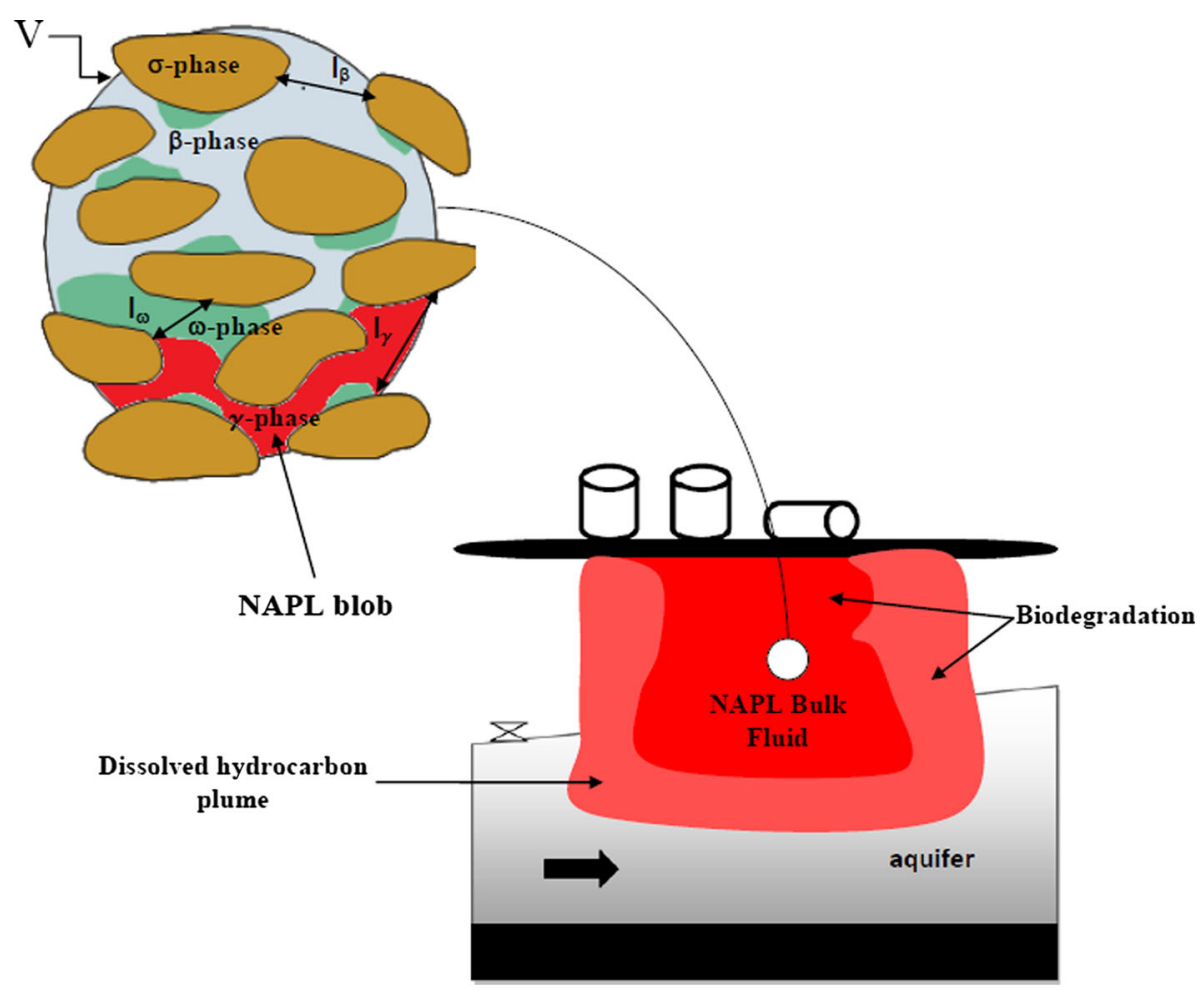

Fig. 1 Sketch of an aquifer contamination by a trapped hydrocarbon phase

validity domain of this model in terms of hydrodynamic and transport conditions (i.e., Péclet and Damköhler numbers).

\section{Pore scale Problem}

The pore scale problem under consideration corresponds to the mass balance of a component $A$ within a three-phase system, respectively, the fluid ( $\beta$-phase), the biofilm ( $\omega$-phase), and the NAPL ( $\gamma$-phase) phases. Water flows through a biofilm-occupied porous medium (the $\sigma$-phase is the solid phase) where NAPL blobs are present. The biofilm is assimilated to a locally homogeneous phase (e.g., Wood and Whitaker 1998). The $\gamma$-phase is assumed trapped residually into the porous matrix and it is immobile (e.g., Quintard and Whitaker $1999,1994)$. In the $\omega$-phase, only diffusion and reaction phenomena take place, while in the $\beta$-phase, solute transport is governed by advection and diffusion. We do not consider here the effect of other physical parameters (temperature and pressure). We restrict our analysis to the simplest possible process in which the $\gamma$-phase consists of a single component (species $A$ ) that is slightly soluble in the $\beta$-phase so that it can be considered as a tracer dissolved in the aqueous phase (e.g., Quintard and Whitaker 1999). It is biodegradable but can be toxic for bacteria at high concentrations. We consider an incompressible fluid flow governed by Stokes equations, and the velocity field is assumed to be known. 
The pore scale boundary value problem under consideration is described by the following microscopic equations

\section{Mass balance equations}

$$
\begin{gathered}
\frac{\partial\left(c_{A \beta}\right)}{\partial t}+\nabla \cdot\left(\mathbf{v}_{A \beta} c_{A \beta}\right)=0 \text { in the } \beta \text {-phase } \\
\frac{\partial\left(c_{A \omega}\right)}{\partial t}+\nabla \cdot\left(\mathbf{v}_{A \omega} c_{A \omega}\right)=R_{A \omega} \text { in the } \omega \text {-phase } \\
\frac{\partial \rho_{\gamma}}{\partial t}=0 \text { in the } \gamma \text {-phase }
\end{gathered}
$$

\section{Momentum equation}

$$
\begin{gathered}
\nabla p_{\beta}=\mu_{\beta} \nabla^{2} \mathbf{v}_{\beta} \text { in the } \beta \text {-phase } \\
\text { B.C. } 1 \quad c_{A \beta}\left(\mathbf{v}_{A \beta}-\mathbf{w}_{\beta \sigma}\right) \cdot \mathbf{n}_{\beta \sigma}=0 \quad \text { at } A_{\beta \sigma} \\
\text { B.C. } 2 \quad c_{A \omega}\left(\mathbf{v}_{A \omega}-\mathbf{w}_{\omega \sigma}\right) \cdot \mathbf{n}_{\omega \sigma}=0 \quad \text { at } A_{\omega \sigma} \\
\text { B.C. } 3 \quad c_{A \beta}=K_{\beta \omega} c_{A \omega} \text { at } A_{\beta \omega} \\
\text { B.C.4 } c_{A \beta}\left(\mathbf{v}_{A \beta}-\mathbf{w}_{\beta \omega}\right) . \mathbf{n}_{\beta \omega}=c_{A \omega}\left(\mathbf{v}_{A \omega}-\mathbf{w}_{\beta \omega}\right) \cdot \mathbf{n}_{\beta \omega} \quad \text { at } A_{\beta \omega} \\
\text { B.C.5 } \quad c_{A \beta}=K_{\beta \gamma} \rho_{\gamma}=c_{A \beta}^{\text {eq }} \text { at } A_{\beta \gamma} \\
\text { B.C.6 } \quad c_{A \omega}=K_{\omega \gamma} \rho_{\gamma}=c_{A \omega}^{\text {eq }} \text { at } A_{\omega \gamma}
\end{gathered}
$$

Here, $c_{A \beta}$ and $c_{A \omega}$ represent the mass concentration of component $A$ (substrate) in the fluid and biofilm phases, respectively; $\rho_{\gamma}$ is the mass concentration of pure species $A$ in the $\gamma$ phase; $\mathbf{v}_{A \beta}$ represents the velocity of component $\mathrm{A}$ in the $\beta$-phase and $\mathbf{v}_{\beta}$ the mass average fluid velocity; $c_{A \beta}^{\mathrm{eq}}$ is the concentration of species $A$ in the $\beta$-phase in equilibrium with $\gamma$; $c_{A \omega}^{\mathrm{eq}}$ is the concentration of species $A$ in the $\omega$-phase in equilibrium with $\gamma ; \mathbf{w}_{\beta \omega}$ represents the velocity of the fluid-biofilm interface $A_{\beta \omega}, \mathbf{w}_{\beta \gamma}, \mathbf{w}_{\beta \sigma}, \mathbf{w}_{\omega \beta}, \mathbf{w}_{\omega \gamma}$, and $\mathbf{w}_{\omega \sigma}$ are similarly defined. $R_{A \omega}$ is a nonlinear kinetic reaction term.

In the presence of NAPL phase, the growth of bacteria liberates biosurfactants and solubilizing agents (Osswald et al. 1996) present only in the biofilm. This mechanism leads to a change in stress tension and hence impact the phase velocities. In addition, the equilibrium partitioning coefficient between the biofilm and NAPL phases $\left(K_{\omega \gamma}\right)$ also depends on the surfactant concentration (Garcia-Junco et al. 2003). Additional momentum equation for the $\gamma$-phase and mass balance equation for the surfactant concentration are theoretically required for describing this complex process. As a first approximation, we assumed: (i) a negligible impact on the NAPL mobility considered as immobile (e.g., Ahmadi et al. 2001; Quintard and Whitaker 1999) and (ii) a constant concentration of biosurfactants in the biofilm phase. Thus, $K_{\omega \gamma}$ is constant over the $\omega-\gamma$ interface. Note that the values of $K_{\beta \gamma}$ and $K_{\omega \gamma}$ may be different to account for the presence of biosurfactants at the $\omega-\gamma$ interface, and we have usually this relation

$$
K_{\beta \gamma} \leq K_{\omega \gamma}
$$




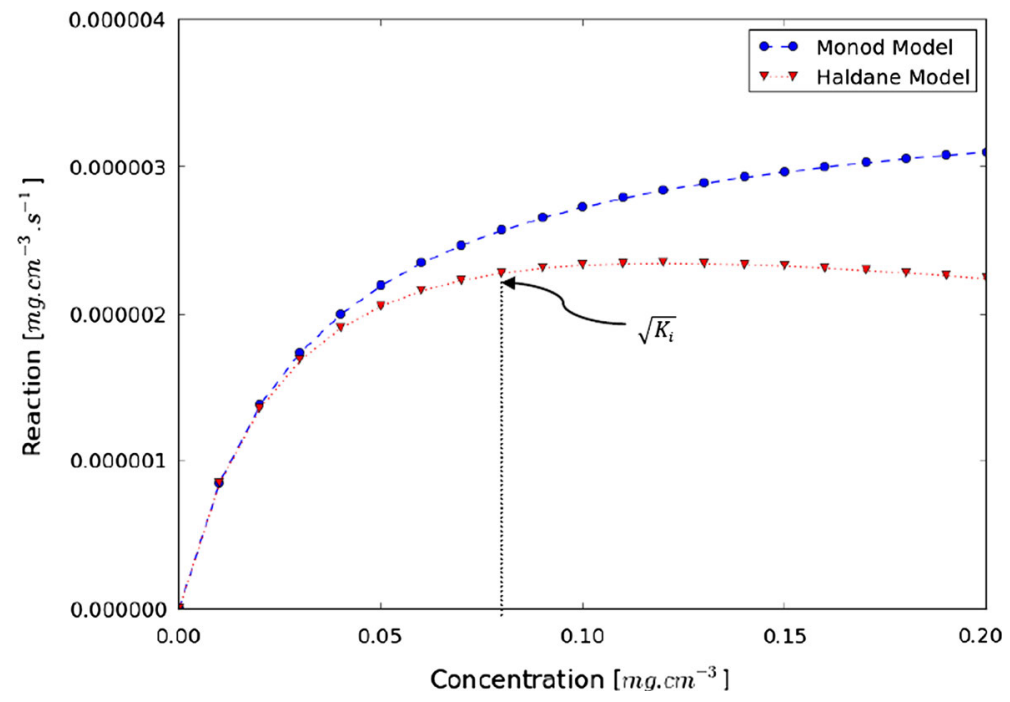

Fig. 2 Comparison of Haldane and Monod kinetics $\left(c_{B \omega}^{*}=1 ; \mu_{A \omega}=3.06 \times 10^{-5} \mathrm{~s}^{-1} ; \rho_{\omega}=0.1 \mathrm{mg} \mathrm{cm}^{-3}\right.$; $K_{A \omega}=3.2 \times 10^{-2} \mathrm{mg} \mathrm{cm}^{-3} ; K_{i}=0.0045 \mathrm{mg} \mathrm{cm}^{-3}$ )

Otherwise, to meet the thermodynamic equilibrium of the system $(\beta, \omega$, and $\gamma)$ at rest, the equilibrium partitioning coefficient between the fluid and biofilm phases $\left(K_{\beta \omega}\right)$ is fixed and respects the relationship

$$
K_{\beta \omega}=\frac{K_{\beta \gamma}}{K_{\omega \gamma}}
$$

In this paper, we use the Haldane model to characterize NAPL biodegradation. This model takes into account for inhibitory effect of substrate concentration on bacterial growth, such as described in the literature (Saravanan et al. 2008). Under these conditions, the reaction term is defined as

$$
\begin{aligned}
R_{A \omega} & =\mu_{A \omega} \rho_{\omega} c_{B \omega}^{*} \frac{c_{A \omega}}{c_{A \omega}+K_{A \omega}+\frac{c_{A \omega}^{2}}{K_{i}}} \\
\text { with: } \quad c_{B \omega}^{*} & =\frac{c_{B \omega}}{c_{B \omega}+K_{B \omega}}
\end{aligned}
$$

A comparison of both kinetics for a given set of parameters is illustrated in Fig. 2. Contrary to Monod kinetics, we observe that the reaction rate for Haldane kinetics decreases with substrate concentration beyond a limit value $\sqrt{K_{i}}$. This value corresponds to the hydrocarbon concentration from which bacterial growth is inhibited.

This model, Eq. (18), considers the system with a single substrate (carbon and energy source) which has been denoted as species $A$, and a single electron acceptor (such as oxygen) which has been denoted as species $B$. For the sake of simplicity, we suppose a large excess of the species $B$ (oxygen) (e.g., Golfier et al. 2009; Orgogozo et al. 2013). As a consequence, the inhibitory effects are not involved in the consumption of the species $B$ and $c_{B \omega}$ is constant. In these equations, $\mu_{A \omega}$ represents the specific degradation rate for the substrate, $\rho_{\omega}$ is the microbial concentration, $K_{A \omega}$ and $K_{B \omega}$ are the half-saturation constants for the substrate and electron acceptor (species $B$ ), and $K_{i}$ is the inhibition constant. Note that $c_{A \omega}$ and $c_{B \omega}$ are 
spatial average concentrations because the biofilm is itself a multiphase system consisting of cells and extracellular material Wood and Whitaker (1998, 2000). In addition, we determine the volumetric rate of dissolution of the NAPL phase. We use the two conditions given by Eqs. (11) and (12) and arrange the form of these equations as

$$
\begin{aligned}
& \mathbf{w}_{\beta \gamma} \cdot \mathbf{n}_{\beta \gamma}=-\frac{1}{\rho_{\gamma}} c_{A \beta}\left(\mathbf{v}_{A \beta}-\mathbf{w}_{\beta \gamma}\right) \cdot \mathbf{n}_{\beta \gamma} \quad \text { at } A_{\beta \gamma} \\
& \mathbf{w}_{\omega \gamma} \cdot \mathbf{n}_{\omega \gamma}=-\frac{1}{\rho_{\gamma}} c_{A \omega}\left(\mathbf{v}_{A \omega}-\mathbf{w}_{\omega \gamma}\right) \cdot \mathbf{n}_{\omega \gamma} \text { at } A_{\omega \gamma}
\end{aligned}
$$

\section{Volume Averaging}

Our analysis of the mass transport problem suggested in Fig. 1 is based on the development of the macroscale equations for species $A$. We follow the developments presented in Quintard and Whitaker (1999) and Golfier et al. (2009) who used the method of volume averaging Whitaker (1999) to derive the macroscale equations.

Referring to the method of volume averaging, we define the superficial average concentration of species $A$ in the $\omega$-phase (biofilm) as

$$
\left\langle c_{A \omega}\right\rangle=\frac{1}{V} \int_{\vartheta_{\omega}(x, t)} c_{A \omega} \mathrm{d} V
$$

with $V$, describing the averaging volume shown in Fig. 1 (the averaging domain is a geometric entity of macroscopic field $\left.\mathbb{V}_{\mathbb{M}}\right)$ and $\vartheta_{\omega}(x, t)$ is the Euclidean space representing the $\omega$-phase contained in the volume $V$. A similar relation holds for the average concentration of species $A$ in the $\beta$-phase,

$$
\left\langle c_{A \beta}\right\rangle=\frac{1}{V} \int_{\vartheta_{\beta}(x, t)} c_{A \beta} \mathrm{d} V
$$

The intrinsic average concentrations for the two phases $(\omega$ and $\beta$ ) are given by

$$
\left\langle c_{A \omega}\right\rangle^{\omega}=\frac{1}{V_{\omega}(x, t)} \int_{\vartheta_{\omega}(x, t)} c_{A \omega} \mathrm{d} V \quad\left\langle c_{A \beta}\right\rangle^{\beta}=\frac{1}{V_{\beta}(x, t)} \int_{\vartheta_{\beta}(x, t)} c_{A \beta} \mathrm{d} V
$$

with $V_{\beta}(x, t)$ and $V_{\omega}(x, t)$ the Lebesgue measures of $\vartheta_{\beta}(x, t)$ and $\vartheta_{\omega}(x, t)$ that are the volumes of the respective phases. Superficial and intrinsic averages given by Eqs. (22) and (23) are related by

$$
\left\langle c_{A \beta}\right\rangle=\epsilon_{\beta}(x, t)\left\langle c_{A \beta}\right\rangle^{\beta} \quad\left\langle c_{A \omega}\right\rangle=\epsilon_{\omega}(x, t)\left\langle c_{A \omega}\right\rangle^{\omega}
$$

where $\epsilon_{\omega}(x, t)$ and $\epsilon_{\beta}(x, t)$ represent the volume fractions of the $\omega$ - and $\beta$-phases, respectively, defined as

$$
\epsilon_{\beta}(x, t)=\frac{V_{\beta}(x, t)}{V} \epsilon_{\omega}(x, t)=\frac{V_{\omega}(x, t)}{V}
$$

In practice, the volume of biofilm $v_{\omega}$ changes in time due to the bacteria growth. However, we assumed in the present study that changes in the $V_{\omega}$ and $V_{\beta}$ volumes are uncoupled from the transport problem due to the disparity of the characteristic timescales. Indeed, the characteristic time for biomass growth (about $10^{4}-10^{6} \mathrm{~s}$ ) is usually much larger than the timescale for substrate transport by diffusion and convection (from $10^{-3}$ to $10^{3} \mathrm{~s}$ ) Picioreanu, van Loosdrecht et al. (2000). This assumption is commonly adopted for this kind of problem 
(e.g., Golfier et al. 2009; Davit et al. 2010). As a consequence, bacterial growth will be neglected hereafter.

In the developments that follow, the phase variables $c_{A \beta}$ and $c_{A \omega}$ can be expressed following Gray's decomposition (Gray et al. 1993)

$$
\begin{gathered}
c_{A \beta}=\left\langle c_{A \beta}\right\rangle^{\beta}+\tilde{c}_{A \beta} \\
c_{A \omega}=\left\langle c_{A \omega}\right\rangle^{\omega}+\tilde{c}_{A \omega}
\end{gathered}
$$

with $\tilde{c}_{A \beta}$ and $\tilde{c}_{A \omega}$ the spatial deviation concentrations in both phases.

We begin our analysis by applying the spatial and temporal averaging theorems that can be expressed as Whitaker (1999)

$$
\begin{aligned}
\left\langle\nabla c_{A \beta}\right\rangle= & \nabla\left\langle c_{A \beta}\right\rangle+\frac{1}{V} \int_{A_{\beta \omega}} \mathbf{n}_{\beta \omega} c_{A \beta} \mathrm{d} A+\frac{1}{V} \int_{A_{\beta \gamma}} \mathbf{n}_{\beta \gamma} c_{A \beta} \mathrm{d} A+\frac{1}{V} \int_{A_{\beta \sigma}} \mathbf{n}_{\beta \sigma} c_{A \beta} \mathrm{d} A \\
\left\langle\nabla \cdot\left(\mathbf{v}_{A \beta} c_{A \beta}\right)\right\rangle= & \nabla \cdot\left\langle\mathbf{v}_{A \beta} c_{A \beta}\right\rangle+\frac{1}{V} \int_{A_{\beta \omega}} \mathbf{n}_{\beta \omega} \cdot\left(\mathbf{v}_{A \beta} c_{A \beta}\right) \mathrm{d} A \\
& +\frac{1}{V} \int_{A_{\beta \gamma}} \mathbf{n}_{\beta \gamma} \cdot\left(\mathbf{v}_{A \beta} c_{A \beta}\right) \mathrm{d} A+\frac{1}{V} \int_{A_{\beta \sigma}} \mathbf{n}_{\beta \sigma} \cdot\left(\mathbf{v}_{A \beta} c_{A \beta}\right) \mathrm{d} A \\
& \left\langle\frac{\partial c_{A \beta}}{\partial t}\right\rangle=\frac{\partial\left\langle c_{A \beta}\right\rangle}{\partial t}-\frac{1}{V} \int_{A_{\beta \omega}} \mathbf{n}_{\beta \omega} \cdot c_{A \beta} \mathbf{w}_{\beta \omega} \mathrm{d} A \\
& -\frac{1}{V} \int_{A_{\beta \gamma}} \mathbf{n}_{\beta \gamma} \cdot c_{A \beta} \mathbf{w}_{\beta \gamma} \mathrm{d} A-\frac{1}{V} \int_{A_{\beta \sigma}} \mathbf{n}_{\beta \sigma} \cdot c_{A \beta} \mathbf{w}_{\beta \sigma} \mathrm{d} A
\end{aligned}
$$

We consider that the solid phase ( $\sigma$-phase) is rigid; therefore, $\mathbf{w}_{i \sigma} \cdot \mathbf{n}_{i \sigma}=0(i=\beta, \omega, \gamma)$. Subsequently, we keep the mass flux at the $\beta-\sigma$ and $\omega-\sigma$ interfaces in equations to simplify the representation of the interfacial flux.

We have used the terminology $A_{\beta}$ to indicate all the surfaces in contact with the $\beta$-phase (i.e., $A_{\beta \omega}, A_{\beta \gamma}$, and $A_{\beta \sigma}$ ). The term $\mathbf{n}_{\beta \omega}$ indicates the unit normal pointing outward from the $\beta$-phase toward the $\omega$-phase; $\mathbf{n}_{\beta \gamma}$ and $\mathbf{n}_{\beta \sigma}$ are similarly defined.

First, we can use the volume-averaging theorems for obtaining the average transport equation in each phase

$\beta$-phase

$$
\begin{aligned}
& \underbrace{\frac{\partial\left(\epsilon_{\beta}\left\langle c_{A \beta}\right\rangle^{\beta}\right)}{\partial t}}_{\text {Accumulation }}+\underbrace{\nabla \cdot\left\langle\mathbf{v}_{A \beta} c_{A \beta}\right\rangle}_{\text {Advection }}+\underbrace{\frac{1}{V} \int_{A_{\beta \gamma}} \mathbf{n}_{\beta \gamma} \cdot c_{A \beta}\left(\mathbf{v}_{A \beta}-\mathbf{w}_{\beta \gamma}\right) \mathrm{d} A}_{\text {Interfacial flux }} \\
& +\underbrace{\frac{1}{V} \int_{A_{\beta \omega}} \mathbf{n}_{\beta \omega} \cdot c_{A \beta}\left(\mathbf{v}_{A \beta}-\mathbf{w}_{\beta \omega}\right) \mathrm{d} A}_{\text {Interfacial flux }}+\underbrace{\frac{1}{V} \int_{A_{\beta \sigma}} \mathbf{n}_{\beta \sigma} \cdot c_{A \beta}\left(\mathbf{v}_{A \beta}-\mathbf{w}_{\beta \sigma}\right) \mathrm{d} A}_{\text {Interfacial flux }}=0
\end{aligned}
$$

$\omega$-phase

$$
\underbrace{\frac{\partial\left(\epsilon_{\omega}\left\langle c_{A \omega}\right\rangle^{\omega}\right)}{\partial t}}_{\text {Accumulation }}+\underbrace{\nabla \cdot\left\langle\mathbf{v}_{A \omega} c_{A \omega}\right\rangle}_{\text {Advection }}+\underbrace{\frac{1}{V} \int_{A_{\omega \gamma}} \mathbf{n}_{\omega \gamma} \cdot c_{A \omega}\left(\mathbf{v}_{A \omega}-\mathbf{w}_{\omega \gamma}\right) \mathrm{d} A}_{\text {Interfacial flux }}
$$




$$
+\underbrace{\frac{1}{V} \int_{A_{\omega \beta}} \mathbf{n}_{\omega \beta} \cdot c_{A \omega}\left(\mathbf{v}_{A \omega}-\mathbf{w}_{\omega \beta}\right) \mathrm{d} A}_{\text {Interfacial flux }}+\underbrace{\frac{1}{V} \int_{A_{\omega \sigma}} \mathbf{n}_{\omega \sigma} \cdot c_{A \omega}\left(\mathbf{v}_{A \omega}-\mathbf{w}_{\omega \sigma}\right) \mathrm{d} A}_{\text {interfacial flux }}=\underbrace{\epsilon_{\omega}\left\langle R_{A \omega}\right\rangle^{\omega}}_{\text {Reaction }}
$$

$\gamma$-phase

$$
\underbrace{\frac{\partial\left\langle\rho_{\gamma}\right\rangle}{\partial t}}_{\text {Accumulation }}+\underbrace{\frac{1}{V} \int_{A_{\beta \gamma}} \rho_{\gamma} \mathbf{n}_{\beta \gamma} \cdot \mathbf{w}_{\beta \gamma} d A+\frac{1}{V} \int_{A_{\omega \gamma}} \rho_{\gamma} \mathbf{n}_{\omega \gamma} \cdot \mathbf{w}_{\omega \gamma} d A}_{\text {Interfacial flux }}=0
$$

The macroscopic reaction term that appear in Eq. (33) can be expressed as (e.g., Wood and Whitaker 1998, 2000)

$$
\left\langle R_{A \omega}\right\rangle^{\omega}=-\mu_{A \omega} \rho_{\omega} c_{B \omega}^{*} \frac{\left\langle c_{A \omega}\right\rangle^{\omega}}{\left\langle c_{A \omega}\right\rangle^{\omega}+K_{A \omega}+\frac{\left(\left\langle c_{A \omega}\right)^{\omega}\right)^{2}}{K_{i}}}
$$

To obtain the last equation, Eq. (35), we have adopted the following assumptions (used only to linearize the nonlinear reaction term)

$$
\tilde{c}_{A \omega}<<\left\langle c_{A \omega}\right\rangle^{\omega}, \tilde{c}_{A \omega}^{2}<<\left(\left\langle c_{A \omega}\right\rangle^{\omega}\right)^{2} .
$$

The constraints associated with Eq. (36) have been previously developed in Wood and Whitaker (2000). Note that, recently, Lugo-Mèndez et al. (2015) have revisited the upscaling process of nonlinear reactive mass transport.

Taking into account the relationships expressing the condition of a dilute solution of species $A$ [additional details are available in Quintard and Whitaker (1999)] and neglecting the advection term in the $\omega$-phase (e.g., Golfier et al. 2009; Orgogozo et al. 2013), the superficial average equations (Eqs. (32), (33)) become

$$
\begin{aligned}
c_{A \beta} \mathbf{v}_{A \beta} & =c_{A \beta} \mathbf{v}_{\beta}-D_{A \beta} \nabla c_{A \beta} \\
c_{A \omega} \mathbf{v}_{A \omega} & =c_{A \omega} \mathbf{v}_{\omega}-D_{A \omega} \nabla c_{A \omega}
\end{aligned}
$$

\section{$\beta$-phase}

$$
\begin{aligned}
& \underbrace{\frac{\partial\left(\epsilon_{\beta}\left\langle c_{A \beta}\right\rangle^{\beta}\right)}{\partial t}}_{\text {Accumulation }}+\underbrace{\nabla \cdot\left\langle\mathbf{v}_{\beta} c_{A \beta}\right\rangle}_{\text {Advection }}=\underbrace{\nabla \cdot\left\langle D_{A \beta} \nabla c_{A \beta}\right\rangle}_{\text {Dispersion }} \\
& -\underbrace{\frac{1}{V} \int_{A_{\beta \gamma}} \mathbf{n}_{\beta \gamma} \cdot\left(c_{A \beta}\left(\mathbf{v}_{\beta}-\mathbf{w}_{\beta \gamma}\right)-D_{A \beta} \nabla c_{A \beta}\right) d A}_{\text {Interfacial flux }} \\
& -\underbrace{\frac{1}{V} \int_{A_{\beta \omega}} \mathbf{n}_{\beta \omega} \cdot\left(c_{A \beta}\left(\mathbf{v}_{\beta}-\mathbf{w}_{\beta \omega}\right)-D_{A \beta} \nabla c_{A \beta}\right) d A}_{\text {Interfacial flux }} \\
& -\underbrace{\frac{1}{V} \int_{A_{\beta \sigma}} \mathbf{n}_{\beta \sigma} \cdot\left(c_{A \beta}\left(\mathbf{v}_{\beta}-\mathbf{w}_{\beta \sigma}\right)-D_{A \beta} \nabla c_{A \beta}\right) d A}_{\text {Interfacial flux }}
\end{aligned}
$$


$\omega$-phase

$$
\begin{aligned}
& \underbrace{\frac{\partial\left(\epsilon_{\omega}\left\langle c_{A \omega}\right\rangle^{\omega}\right)}{\partial t}}_{\text {Accumulation }}=\underbrace{\nabla \cdot\left\langle\mathbf{D}_{\mathbf{A} \omega} \cdot \nabla c_{A \omega}\right\rangle}_{\text {Dispersion }} \\
& -\underbrace{\frac{1}{V} \int_{A_{\omega \gamma}} \mathbf{n}_{\omega \gamma} \cdot\left(c_{A \omega}\left(\mathbf{v}_{\omega}-\mathbf{w}_{\omega \gamma}\right)-\mathbf{D}_{\mathbf{A} \omega} \cdot \nabla c_{A \omega}\right) \mathrm{d} A}_{\text {Interfacial flux }} \\
& -\underbrace{\frac{1}{V} \int_{A_{\omega \beta}} \mathbf{n}_{\omega \beta} \cdot\left(c_{A \beta}\left(\mathbf{v}_{\omega}-\mathbf{w}_{\omega \beta}\right)-\mathbf{D}_{\mathbf{A} \omega} \cdot \nabla c_{A \omega}\right) \mathrm{d} A}_{\text {Interfacial flux }} \\
& -\underbrace{\frac{1}{V} \int_{A_{\omega \sigma}} \mathbf{n}_{\omega \sigma} \cdot\left(c_{A \omega}\left(\mathbf{v}_{\omega}-\mathbf{w}_{\omega \sigma}\right)-\mathbf{D}_{\mathbf{A} \omega} \cdot \nabla c_{A \omega}\right) \mathrm{d} A}+\underbrace{\epsilon_{\omega}\left\langle R_{A \omega}\right\rangle^{\omega}}_{\text {Reaction }}
\end{aligned}
$$

where $D_{A \beta}$ is the diffusion coefficient for the $A$ component in the fluid and $\mathbf{D}_{A \omega}$ describing the effective diffusion tensor of component $A$ in the biofilm phase. Using Gray's spatial decomposition into the divergence term, we obtain

$$
\begin{aligned}
& \frac{\partial\left(\epsilon_{\beta}\left\langle c_{A \beta}\right\rangle^{\beta}\right)}{\partial t}+\nabla \cdot\left(\epsilon_{\beta}\left\langle\mathbf{v}_{\beta}\right\rangle^{\beta}\left\langle c_{A \beta}\right\rangle^{\beta}\right)+\nabla \cdot\left\langle\tilde{\mathbf{v}}_{\beta} \tilde{c}_{A \beta}\right\rangle \\
& =\nabla \cdot\left\langle D_{A \beta} \nabla c_{A \beta}\right\rangle-\frac{1}{V} \int_{A_{\beta \gamma}} \mathbf{n}_{\beta \gamma} \cdot\left(c_{A \beta}\left(\mathbf{v}_{\beta}-\mathbf{w}_{\beta \gamma}\right)-D_{A \beta} \nabla c_{A \beta}\right) \mathrm{d} A \\
& -\frac{1}{V} \int_{A_{\beta \omega}} \mathbf{n}_{\beta \omega} \cdot\left(c_{A \beta}\left(\mathbf{v}_{\beta}-\mathbf{w}_{\beta \omega}\right)-D_{A \beta} \nabla c_{A \beta}\right) \mathrm{d} A-\frac{1}{V} \int_{A_{\beta \sigma}} \mathbf{n}_{\beta \sigma} \cdot\left(c_{A \beta}\left(\mathbf{v}_{\beta}-\mathbf{w}_{\beta \sigma}\right)\right. \\
& \left.\quad-D_{A \beta} \nabla c_{A \beta}\right) \mathrm{d} A
\end{aligned}
$$

Now considering that the molecular diffusivity in the $\beta$-phase is constant (e.g., Quintard and Whitaker 1994, 1999; Wood and Whitaker 2000) and using the averaging theorem, the diffusive term can be developed as

$$
\begin{aligned}
\left\langle D_{A \beta} \nabla c_{A \beta}\right\rangle= & D_{A \beta}\left[\epsilon_{\beta} \nabla\left\langle c_{A \beta}\right\rangle^{\beta}+\nabla \epsilon_{\beta}\left\langle c_{A \beta}\right\rangle^{\beta}+\frac{1}{V} \int_{A_{\beta \omega}} \mathbf{n}_{\beta \omega}\left\langle c_{A \beta}\right\rangle^{\beta} \mathrm{d} A\right. \\
& +\frac{1}{V} \int_{A_{\beta \gamma}} \mathbf{n}_{\beta \gamma}\left\langle c_{A \beta}\right\rangle^{\beta} d A+\frac{1}{V} \int_{A_{\beta \sigma}} \mathbf{n}_{\beta \sigma}\left\langle c_{A \beta}\right\rangle^{\beta} \mathrm{d} A \\
& \left.+\frac{1}{V} \int_{A_{\beta \omega}} \mathbf{n}_{\beta \omega} \tilde{c}_{A \beta} \mathrm{d} A+\frac{1}{V} \int_{A_{\beta \gamma}} \mathbf{n}_{\beta \gamma} \tilde{c}_{A \beta} \mathrm{d} A+\frac{1}{V} \int_{A_{\beta \sigma}} \mathbf{n}_{\beta \sigma} \tilde{c}_{A \beta} \mathrm{d} A\right]
\end{aligned}
$$

Applying the geometric lemma Quintard and Whitaker 1994,

$$
\nabla \epsilon_{\beta}=-\frac{1}{V} \int_{A_{\beta \omega}} \mathbf{n}_{\beta \omega} d A-\frac{1}{V} \int_{A_{\beta \gamma}} \mathbf{n}_{\beta \gamma} \mathrm{d} A-\frac{1}{V} \int_{A_{\beta \sigma}} \mathbf{n}_{\beta \sigma} \mathrm{d} A
$$

The diffusive flux can be rewritten as

$$
\begin{aligned}
\left\langle D_{A \beta} \nabla c_{A \beta}\right\rangle= & D_{A \beta}\left(\epsilon_{\beta} \nabla\left\langle c_{A \beta}\right\rangle^{\beta}+\frac{1}{V} \int_{A_{\beta \omega}} \mathbf{n}_{\beta \omega} \tilde{c}_{A \beta} \mathrm{d} A+\frac{1}{V} \int_{A_{\beta \gamma}} \mathbf{n}_{\beta \gamma} \tilde{c}_{A \beta} \mathrm{d} A\right. \\
& \left.+\frac{1}{V} \int_{A_{\beta \sigma}} \mathbf{n}_{\beta \sigma} \tilde{c}_{A \beta} \mathrm{d} A\right)
\end{aligned}
$$


The previous result (Eq. (44)) is associated with the two following length-scale constraints (Quintard and Whitaker 1994)

$$
l_{\beta}<<r_{o} \quad r_{o}^{2}<<L_{c}^{2}
$$

where $r_{o}$ is the radius of the averaging volume, $l_{\beta}$ is the characteristic length associated with the $\beta$-phase, and $L_{c}$ is the characteristic length defining the macroscale. At this point, introducing Eq. (44) into Eq. (41) leads to

$$
\begin{aligned}
& \frac{\partial\left(\epsilon_{\beta}\left\langle c_{A \beta}\right\rangle^{\beta}\right)}{\partial t}+\nabla \cdot\left(\epsilon_{\beta}\left\langle\mathbf{v}_{\beta}\right\rangle^{\beta}\left\langle c_{A \beta}\right\rangle^{\beta}\right)+\nabla \cdot\left\langle\tilde{\mathbf{v}}_{\beta} \tilde{c}_{A \beta}\right\rangle \\
& =\nabla \cdot\left[D_{A \beta}\left(\epsilon_{\beta} \nabla\left\langle c_{A \beta}\right\rangle^{\beta}+\frac{1}{V} \int_{A_{\beta \omega}} \mathbf{n}_{\beta \omega} \tilde{c}_{A \beta} \mathrm{d} A+\frac{1}{V} \int_{A_{\beta \gamma}} \mathbf{n}_{\beta \gamma} \tilde{c}_{A \beta} \mathrm{d} A+\frac{1}{V} \int_{A_{\beta \sigma}} \mathbf{n}_{\beta \sigma} \tilde{c}_{A \beta} \mathrm{d} A\right)\right] \\
& -\frac{1}{V} \int_{A_{\beta \gamma}} \mathbf{n}_{\beta \gamma} \cdot\left(c_{A \beta}\left(\mathbf{v}_{\beta}-\mathbf{w}_{\beta \gamma}\right)-D_{A \beta} \nabla c_{A \beta}\right) \mathrm{d} A-\frac{1}{V} \int_{A_{\beta \omega}} \mathbf{n}_{\beta \omega} \cdot\left(c _ { A \beta } \left(\mathbf{v}_{\beta}\right.\right. \\
& \left.\left.-\mathbf{w}_{\beta \omega}\right)-D_{A \beta} \nabla c_{A \beta}\right) \mathrm{d} A-\frac{1}{V} \int_{A_{\beta \sigma}} \mathbf{n}_{\beta \sigma} \cdot\left(c_{A \beta}\left(\mathbf{v}_{\beta}-\mathbf{w}_{\beta \sigma}\right)-D_{A \beta} \nabla c_{A \beta}\right) \mathrm{d} A
\end{aligned}
$$

This result requires the use of the approximation (Carbonell and Whitaker 1983)

$$
\left\langle\tilde{c}_{A \beta}\right\rangle^{\beta}=0 \quad\left\langle\tilde{\mathbf{v}}_{\beta}\right\rangle^{\beta}=0
$$

Finally, using again the Gray's decomposition in the integral terms on the right-hand side of Eqs. (46), Eq. (46) can be expressed as

$$
\begin{aligned}
& \frac{\partial\left(\epsilon_{\beta}\left\langle c_{A \beta}\right\rangle^{\beta}\right)}{\partial t}+\nabla \cdot\left(\epsilon_{\beta}\left\langle\mathbf{v}_{\beta}\right\rangle^{\beta}\left\langle c_{A \beta}\right\rangle^{\beta}\right)+\nabla \cdot\left\langle\tilde{\mathbf{v}}_{\beta} \tilde{c}_{A \beta}\right\rangle \\
& =\epsilon_{\beta} \nabla \cdot\left(D_{A \beta} \nabla\left\langle c_{A \beta}\right\rangle^{\beta}\right)+\left(\nabla \epsilon_{\beta}\right) \cdot D_{A \beta} \nabla\left\langle c_{A \beta}\right\rangle^{\beta} \\
& \left.+\nabla \cdot\left[\int_{A \beta} \frac{1}{V} \int_{A_{\beta \omega}} \mathbf{n}_{\beta \omega} \tilde{c}_{A \beta} \mathrm{d} A+\frac{1}{V} \int_{A_{\beta \gamma}} \mathbf{n}_{\beta \gamma} \tilde{c}_{A \beta} \mathrm{d} A+\frac{1}{V} \int_{A_{\beta \sigma}} \mathbf{n}_{\beta \sigma} \tilde{c}_{A \beta} \mathrm{d} A\right)\right] \\
& -\frac{1}{V} \int_{A_{\beta \gamma}} \mathbf{n}_{\beta \gamma} \cdot\left(\mathbf{v}_{\beta}-\mathbf{w}_{\beta \gamma}\right) \mathrm{d} A\left\langle c_{A \beta}\right\rangle^{\beta}-\frac{1}{V} \int_{A_{\beta \omega}} \mathbf{n}_{\beta \omega} \cdot\left(\mathbf{v}_{\beta}-\mathbf{w}_{\beta \omega}\right) \mathrm{d} A\left\langle c_{A \beta}\right\rangle^{\beta} \\
& -\frac{1}{V} \int_{A_{\beta \sigma}} \mathbf{n}_{\beta \sigma} \cdot\left(\mathbf{v}_{\beta}-\mathbf{w}_{\beta \sigma}\right) \mathrm{d} A\left\langle c_{A \beta}\right\rangle^{\beta}-\left(\nabla \epsilon_{\beta}\right) \cdot D_{A \beta} \nabla\left\langle c_{A \beta}\right\rangle^{\beta} \\
& -\frac{1}{V} \int_{A_{\beta \gamma}} \mathbf{n}_{\beta \gamma} \cdot \tilde{c}_{A \beta}\left(\mathbf{v}_{\beta}-\mathbf{w}_{\beta \gamma}\right) \mathrm{d} A \\
& -\frac{1}{V} \int_{A_{\beta \omega}} \mathbf{n}_{\beta \omega} \cdot \tilde{c}_{A \beta}\left(\mathbf{v}_{\beta}-\mathbf{w}_{\beta \omega}\right) \mathrm{d} A-\frac{1}{V} \int_{A_{\beta \sigma}} \mathbf{n}_{\beta \sigma} \cdot \tilde{c}_{A \beta}\left(\mathbf{v}_{\beta}-\mathbf{w}_{\beta \sigma}\right) \mathrm{d} A \\
& +\frac{1}{V} \int_{A_{\beta \gamma}} \mathbf{n}_{\beta \gamma} \cdot D_{A \beta} \nabla \tilde{c}_{A \beta} \mathrm{d} A \\
& +\frac{1}{V} \int_{A_{\beta \omega}} \mathbf{n}_{\beta \omega} \cdot D_{A \beta} \nabla \tilde{c}_{A \beta} \mathrm{d} A+\frac{1}{V} \int_{A_{\beta \sigma}} \mathbf{n}_{\beta \sigma} \cdot D_{A \beta} \nabla \tilde{c}_{A \beta} \mathrm{d} A
\end{aligned}
$$

To develop a simplified form of Eq. (48), we need to make use of the total continuity equation (mass balance) in the $\beta$-phase. The total continuity equation, routinely used in the solution 
of liquid-phase flow problems (e.g., Quintard and Whitaker 1999; Soulaine et al. 2011), is expressed as

$$
\frac{\partial \rho_{\beta}}{\partial t}+\nabla \cdot\left(\rho_{\beta} \mathbf{v}_{\beta}\right)=0
$$

Supposing that the variations in $\rho_{\beta}$ are negligible compared to $\mathbf{v}_{\beta}$, we apply the volumeaveraging theorems to the continuity equation, Eq. (49), which leads to

$$
\begin{aligned}
& \frac{\partial \epsilon_{\beta}}{\partial t}+\nabla \cdot\left\langle\mathbf{v}_{\beta}\right\rangle+\frac{1}{V} \int_{A_{\beta \gamma}} \mathbf{n}_{\beta \gamma} \cdot\left(\mathbf{v}_{\beta}-\mathbf{w}_{\beta \gamma}\right) \mathrm{d} A+\frac{1}{V} \int_{A_{\beta \omega}} \mathbf{n}_{\beta \omega} \cdot\left(\mathbf{v}_{\beta}-\mathbf{w}_{\beta \omega}\right) \mathrm{d} A \\
& +\frac{1}{V} \int_{A_{\beta \sigma}} \mathbf{n}_{\beta \sigma} \cdot\left(\mathbf{v}_{\beta}-\mathbf{w}_{\beta \sigma}\right) \mathrm{d} A=0
\end{aligned}
$$

Substituting the average of total continuity equation, Eq. (50), into Eq. (48) gives the unclosed form of the averaged equation in the $\beta$-phase

$$
\begin{aligned}
& \underbrace{\frac{\partial\left\langle c_{A \beta}\right\rangle^{\beta}}{\partial t}}_{\text {Accumulation }}+\underbrace{\left\langle\mathbf{v}_{\beta}\right\rangle^{\beta} \cdot \nabla\left\langle c_{A \beta}\right\rangle^{\beta}}_{\text {Advection }}+\underbrace{\epsilon_{\beta}^{-1} \nabla \cdot\left\langle\tilde{\mathbf{v}}_{\beta} \tilde{c}_{A \beta}\right\rangle}_{\text {Dispersive transport }}=\underbrace{\nabla \cdot\left(D_{A \beta} \nabla\left\langle c_{A \beta}\right\rangle^{\beta}\right)}_{\text {Dispersion }} \\
& +\underbrace{\epsilon_{\beta}^{-1} \nabla \cdot\left[D_{A \beta}\left(\frac{1}{V} \int_{A_{\beta \omega}} \mathbf{n}_{\beta \omega} \tilde{c}_{A \beta} \mathrm{d} A+\frac{1}{V} \int_{A_{\beta \gamma}} \mathbf{n}_{\beta \gamma} \tilde{c}_{A \beta} \mathrm{d} A+\frac{1}{V} \int_{A_{\beta \sigma}} \mathbf{n}_{\beta \sigma} \tilde{c}_{A \beta} \mathrm{d} A\right)\right]}_{\text {Dispersion }} \\
& -\underbrace{\frac{\epsilon_{\beta}^{-1}}{V} \int_{A_{\beta \gamma}} \mathbf{n}_{\beta \gamma} \cdot \tilde{c}_{A \beta}\left(\mathbf{v}_{\beta}-\mathbf{w}_{\beta \gamma}\right) \mathrm{d} A-\frac{\epsilon_{\beta}^{-1}}{V} \int_{A_{\beta \omega}} \mathbf{n}_{\beta \omega} \cdot \tilde{c}_{A \beta}\left(\mathbf{v}_{\beta}-\mathbf{w}_{\beta \omega}\right) \mathrm{d} A}_{\text {Interfacial flux }} \\
& -\underbrace{\frac{\epsilon_{\beta}^{-1}}{V} \int_{A_{\beta \sigma}} \mathbf{n}_{\beta \sigma} \cdot \tilde{c}_{A \beta}\left(\mathbf{v}_{\beta}-\mathbf{w}_{\beta \sigma}\right) \mathrm{d} A+\frac{\epsilon_{\beta}^{-1}}{V} \int_{A_{\beta \gamma}} \mathbf{n}_{\beta \gamma} \cdot D_{A \beta} \nabla \tilde{c}_{A \beta} \mathrm{d} A}_{\text {Interfacial flux }} \\
& +\underbrace{\epsilon_{\beta}^{-1} \mathbf{n}_{\beta \omega} \cdot D_{A \beta} \nabla \tilde{c}_{A \beta} \mathrm{d} A+\int_{A_{\beta \sigma}} \mathbf{n}_{\beta \sigma} \cdot D_{A \beta} \nabla \tilde{c}_{A \beta} \mathrm{d} A}_{A_{\beta \omega}^{-1}}
\end{aligned}
$$

A similar development gives the unclosed form of the averaged equation in the $\omega$-phase

$$
\begin{aligned}
& \underbrace{\frac{\partial\left\langle c_{A \omega}\right\rangle^{\omega}}{\partial t}}_{\text {Accumulation }}=\underbrace{\nabla \cdot\left(\mathbf{D}_{\mathbf{A} \omega} \cdot \nabla\left\langle c_{A \omega}\right\rangle^{\omega}\right)}_{\text {Dispersion }} \\
& +\underbrace{\epsilon_{\omega}^{-1} \nabla \cdot\left[\mathbf{D}_{\mathbf{A} \omega} \cdot\left(\frac{1}{V} \int_{A_{\omega \beta}} \mathbf{n}_{\omega \beta} \tilde{c}_{A \omega} \mathrm{d} A+\frac{1}{V} \int_{A_{\omega \gamma}} \mathbf{n}_{\omega \gamma} \tilde{c}_{A \omega} \mathrm{d} A+\frac{1}{V} \int_{A_{\omega \sigma}} \mathbf{n}_{\beta \sigma} \tilde{c}_{A \omega} \mathrm{d} A\right)\right]}_{\text {Dispersion }} \\
& -\underbrace{\frac{\epsilon_{\omega}^{-1}}{V} \int_{A_{\omega \gamma}} \mathbf{n}_{\omega \gamma} \cdot \tilde{c}_{A \omega}\left(\mathbf{v}_{\omega}-\mathbf{w}_{\omega \gamma}\right) \mathrm{d} A-\frac{\epsilon_{\omega}^{-1}}{V} \int_{A_{\omega \beta}} \mathbf{n}_{\omega \beta} \cdot \tilde{c}_{A \omega}\left(\mathbf{v}_{\omega}-\mathbf{w}_{\omega \beta}\right) \mathrm{d} A}_{\text {Interfacial flux }}
\end{aligned}
$$




$$
\begin{aligned}
& -\underbrace{\frac{\epsilon_{\omega}^{-1}}{V} \int_{A_{\omega \sigma}} \mathbf{n}_{\omega \sigma} \cdot \tilde{c}_{A \omega}\left(\mathbf{v}_{\omega}-\mathbf{w}_{\omega \sigma}\right) \mathrm{d} A+\frac{\epsilon_{\omega}^{-1}}{V} \int_{A_{\omega \gamma}} \mathbf{n}_{\omega \gamma} \cdot \mathbf{D}_{\mathbf{A} \omega} \cdot \nabla \tilde{c}_{A \omega} \mathrm{d} A}_{\text {Interfacial flux }} \\
& +\underbrace{\left.\frac{\epsilon_{\omega}^{-1}}{V} \int_{A_{\omega \beta}} \mathbf{n}_{\omega \beta} \cdot \mathbf{D}_{\mathbf{A} \omega} \cdot \nabla \tilde{c}_{A \omega} \mathrm{d} A+\frac{\epsilon_{\omega}^{-1}}{V} \int_{A_{\omega \sigma}} \mathbf{n}_{\omega \sigma} \cdot \mathbf{D}_{\mathbf{A} \omega} \cdot \nabla \tilde{c}_{A \omega} \mathrm{d} A\right]}_{\text {Interfacial flux }}+\underbrace{\left\langle R_{A \omega}\right\rangle^{\omega}}_{\text {Reaction }}
\end{aligned}
$$

For a binary system and under the dilute solution approximation (Quintard and Whitaker 1999), the velocities of interfaces $\beta-\gamma$ and $\omega-\gamma$ (Eqs. (20), (21)) can be expressed as

$$
\begin{aligned}
& \mathbf{w}_{\beta \gamma} \cdot \mathbf{n}_{\beta \gamma}=\frac{1}{\rho_{\gamma}} \mathbf{n}_{\beta \gamma} \cdot D_{A \beta} \nabla c_{A \beta} \quad \text { at } A_{\beta \gamma} \\
& \mathbf{w}_{\omega \gamma} \cdot \mathbf{n}_{\omega \gamma}=\frac{1}{\rho_{\gamma}} \mathbf{n}_{\omega \gamma} \cdot \mathbf{D}_{\mathbf{A} \omega} \cdot \nabla c_{A \omega} \text { at } A_{\omega \gamma}
\end{aligned}
$$

These results assume that the temperature and pressure gradients are small enough so that the following conditions are satisfied (Quintard and Whitaker 1999)

$$
\begin{aligned}
& c_{A \beta}\left(\mathbf{v}_{\beta}-\mathbf{w}_{\beta \gamma}\right) \cdot \mathbf{n}_{\beta \gamma}=0 \\
& c_{A \omega}\left(\mathbf{v}_{\omega}-\mathbf{w}_{\omega \gamma}\right) \cdot \mathbf{n}_{\omega \gamma}=0
\end{aligned}
$$

Finally, substituting Eqs. (53) and (54) into Eq. (34) yields

$$
\underbrace{\frac{\partial \epsilon_{\gamma}\left\langle\rho_{\gamma}\right\rangle^{\gamma}}{\partial t}}_{\text {Accumulation }}+\underbrace{\frac{1}{V} \int_{A_{\beta \gamma}} \mathbf{n}_{\beta \gamma} \cdot D_{A \beta} \nabla c_{A \beta} \mathrm{d} A+\frac{1}{V} \int_{A_{\omega \gamma}} \mathbf{n}_{\omega \gamma} \cdot \mathbf{D}_{\mathbf{A} \omega} \cdot \nabla c_{A \omega} \mathrm{d} A}_{\text {Interfacial flux }}=0
$$

In this paper, we suppose the NAPL density $\rho_{\gamma}$-constant within the averaging volume $\mathrm{V}$ ( $\rho_{\gamma}=\left\langle\rho_{\gamma}\right\rangle^{\gamma}$, but it does not imply that $\left\langle\rho_{\gamma}\right\rangle$ is constant) so that the last equation can be rewritten as

$$
\begin{aligned}
& \rho_{\gamma} \frac{\partial \epsilon_{\gamma}}{\partial t}+\frac{1}{V} \int_{A_{\beta \gamma}} \mathbf{n}_{\beta \gamma} \cdot D_{A \beta} \nabla\left\langle c_{A \beta}\right\rangle^{\beta} \mathrm{d} A+\frac{1}{V} \int_{A_{\beta \gamma}} \mathbf{n}_{\beta \gamma} \cdot D_{A \beta} \nabla \tilde{c}_{A \beta} \mathrm{d} A \\
& +\frac{1}{V} \int_{A_{\omega \gamma}} \mathbf{n}_{\omega \gamma} \cdot \mathbf{D}_{\mathbf{A} \omega} \cdot \nabla\left\langle c_{A \omega}\right\rangle^{\omega} \mathrm{d} A+\frac{1}{V} \int_{A_{\omega \gamma}} \mathbf{n}_{\omega \gamma} \cdot \mathbf{D}_{\mathbf{A} \omega} \cdot \nabla \tilde{c}_{A \omega} \mathrm{d} A=0
\end{aligned}
$$

\section{Local Mass Equilibrium}

\subsection{The Two-Equation Model}

At this point, we get a set of three unclosed macroscopic equations. We can take advantage of simplifying assumptions for reducing the number of balance equations governing the solute transport at the macroscale. In particular, the local mass equilibrium assumption, referring to conditions close to thermodynamic equilibrium, yields to define a single weighted average concentration for the phases at equilibrium. It must be emphasized that the local mass equilibrium assumption does not imply a zero gradient everywhere, but assumes the presence of sufficiently low-concentration gradients at the microscopic scale whatever the 
phase considered. Assuming small concentration gradients at the fluid-NAPL (or biofilmNAPL) interface is probably too restrictive for many applications where non-equilibrium conditions are classically encountered. On the contrary, considering the hypothesis of local mass equilibrium at the fluid-biofilm interface could be more convenient since this assumption has been already used with success for the dissolved hydrocarbon plume (Golfier et al. 2009). As a consequence, we will adopt this approach in the following, and we refer the reader to Golfier et al. (2009) and Orgogozo et al. (2013) for additional details about this assumption.

At the $\beta-\omega$ interface, this hypothesis is expressed as

$$
\left\langle c_{A \beta}\right\rangle^{\beta}=K_{\beta \omega}\left\langle c_{A \omega}\right\rangle^{\omega}
$$

For analysis purposes, we define an equilibrium weighted average concentration (e.g., Golfier et al. 2009)

$$
\left\{c_{A}\right\}=\left(\frac{\epsilon_{\beta}}{\epsilon_{\omega}+\epsilon_{\beta}}\right)\left\langle c_{A \beta}\right\rangle^{\beta}+\left(\frac{\epsilon_{\omega}}{\epsilon_{\omega}+\epsilon_{\beta}}\right) K_{\beta \omega}\left\langle c_{A \omega}\right\rangle^{\omega}
$$

that possesses the following property:

$$
\left\{c_{A}\right\}=\left\langle c_{A \beta}\right\rangle^{\beta}=K_{\beta \omega}\left\langle c_{A \omega}\right\rangle^{\omega}
$$

The constraints associated with Eq. (61) have been previously developped in Wood et al. (2011) and recalled by Golfier et al. (2009). Under this assumption, the concentrations predicted by Eqs. (51) and (52) can be added to obtain a new equation describing solute transport in the two phases ( $\beta$ - and $\omega$-phases). Summing up these equations eliminates the interfacial flux terms defined on $A_{\beta \omega}$. This new equation, coupled with the mass balance equation for the $\gamma$-phase, (Eq. (58)), has the following form

$$
\begin{aligned}
& \underbrace{\frac{\partial}{\partial t}\left(\left(\epsilon_{\beta}+K_{\beta \omega}^{-1} \epsilon_{\omega}\right)\right)\left\langle c_{A \beta}\right\rangle^{\beta}}_{\text {Accumulation }}+\underbrace{\epsilon_{\beta}\left\langle\mathbf{v}_{\beta}\right\rangle^{\beta} \cdot \nabla\left\langle c_{A \beta}\right\rangle^{\beta}}_{\text {Advection }}=\underbrace{\left(D_{A \beta} \mathbf{I}+K_{\beta \omega}^{-1} \mathbf{D}_{A \omega}\right) \cdot \nabla\left\langle c_{A \beta}\right\rangle^{\beta}}_{\text {Conventional diffusion term }} \\
& +\underbrace{\nabla \cdot\left[\mathbf{X}_{A \beta}+\mathbf{X}_{A \omega}\right]}_{\text {Hydrodynamic dispersion }}-\underbrace{\frac{1}{V} \int_{A_{\beta \gamma}} \mathbf{n}_{\beta \gamma} \cdot \tilde{c}_{A \beta}\left(\mathbf{v}_{\beta}-\mathbf{w}_{\beta \gamma}\right) \mathrm{d} A+\frac{1}{V} \int_{A_{\beta \gamma}} \mathbf{n}_{\beta \gamma} \cdot D_{A \beta} \nabla \tilde{c}_{A \beta} \mathrm{d} A}_{\text {Interfacial flux }} \\
& -\underbrace{\frac{1}{V} \int_{A_{\omega \gamma}} \mathbf{n}_{\omega \gamma} \cdot \tilde{c}_{A \omega}\left(\mathbf{v}_{\omega}-\mathbf{w}_{\omega \gamma}\right) \mathrm{d} A+\frac{1}{V} \int_{A_{\omega \gamma}} \mathbf{n}_{\omega \gamma} \cdot \mathbf{D}_{\mathbf{A} \omega} \cdot \nabla \tilde{c}_{A \omega} \mathrm{d} A} \\
& \text { Interfacial flux } \\
& \underbrace{-\epsilon_{\omega} \mu_{A \omega} \rho_{\omega} c_{B \omega}^{*} \frac{\left\langle c_{A \beta}\right\rangle^{\beta}}{\left\langle c_{A \beta}\right\rangle^{\beta}+K_{\beta \omega} K_{A \omega}+\frac{K_{\beta \omega}^{-1}\left(\left\langle c_{A \beta}\right\rangle^{\beta}\right)^{2}}{K_{i}}}}_{\text {Reaction }}
\end{aligned}
$$

where

$$
\begin{aligned}
\mathbf{X}_{A \beta}= & D_{A \beta} \mathbf{I} .\left(\left[\frac{1}{V} \int_{A_{\beta \omega}} \mathbf{n}_{\beta \omega} \tilde{c}_{A \beta} \mathrm{d} A+\frac{1}{V} \int_{A_{\beta \gamma}} \mathbf{n}_{\beta \gamma} \tilde{c}_{A \beta} \mathrm{d} A+\frac{1}{V} \int_{A_{\beta \sigma}} \mathbf{n}_{\beta \sigma} \tilde{c}_{A \beta} \mathrm{d} A\right]\right) \\
& -\left\langle\tilde{\mathbf{v}}_{\beta} \tilde{c}_{A \beta}\right\rangle
\end{aligned}
$$




$$
\mathbf{X}_{A \omega}=\mathbf{D}_{A \omega} \cdot\left(\frac{1}{V} \int_{A_{\omega \beta}} \mathbf{n}_{\omega \beta} \tilde{c}_{A \omega} \mathrm{d} A+\frac{1}{V} \int_{A_{\omega \gamma}} \mathbf{n}_{\omega \gamma} \tilde{c}_{A \omega} \mathrm{d} A+\frac{1}{V} \int_{A_{\omega \sigma}} \mathbf{n}_{\omega \sigma} \tilde{c}_{A \omega} \mathrm{d} A\right)
$$

These forms of the macroscopic equations (Eqs. (62), (58)) are still unclosed because they contain the quantities $\tilde{c}_{A \beta}$ and $\tilde{c}_{A \omega}$ as independent variables in the area integrals. An estimate for the concentration deviations has to be found.

\section{Closure}

\subsection{Deviations Problem}

In this section, we develop the relationships between spatial deviations and average quantities in order to close the macroscopic model.

We first recall our original differential equations defined in the $\beta$ - and $\omega$-phases at the pore scale

$$
\begin{aligned}
& \frac{\partial\left(c_{A \beta}\right)}{\partial t}+\nabla .\left(\mathbf{v}_{\beta} c_{A \beta}\right)=\nabla \cdot\left(D_{A \beta} \nabla c_{A \beta}\right) \\
& \frac{\partial\left(c_{A \omega}\right)}{\partial t}+\nabla .\left(\mathbf{v}_{\omega} c_{A \omega}\right)=\nabla .\left(\mathbf{D}_{A \omega} . \nabla c_{A \omega}\right)
\end{aligned}
$$

By subtracting Eq. (51) from Eqs. (64) and (52) from Eq. (65), and under conditions of local mass equilibrium (Eq. (59)), we obtain the set of equations governing the concentration deviations (additional details are shown in "Appendix 1")

$$
\begin{aligned}
& \mathbf{v}_{\beta} \cdot \nabla \tilde{c}_{A \beta}+\tilde{\mathbf{v}}_{\beta} \cdot \nabla\left\langle c_{A \beta}\right\rangle^{\beta}=\nabla \cdot\left(D_{A \beta} \nabla \tilde{c}_{A \beta}\right)+\frac{\epsilon_{\beta}^{-1}}{V} \int_{A_{\beta \gamma}} \mathbf{n}_{\beta \gamma} \cdot \tilde{c}_{A \beta}\left(\mathbf{v}_{\beta}-\mathbf{w}_{\beta \gamma}\right) \mathrm{d} A \\
& +\frac{\epsilon_{\beta}^{-1}}{V} \int_{A_{\beta \omega}} \mathbf{n}_{\beta \omega} \cdot \tilde{c}_{A \beta}\left(\mathbf{v}_{\beta}-\mathbf{w}_{\beta \omega}\right) \mathrm{d} A+\frac{\epsilon_{\beta}^{-1}}{V} \int_{A_{\beta \sigma}} \mathbf{n}_{\beta \sigma} \cdot \tilde{c}_{A \beta}\left(\mathbf{v}_{\beta}-\mathbf{w}_{\beta \sigma}\right) \mathrm{d} A \\
& -\frac{\epsilon_{\beta}^{-1}}{V} \int_{A_{\beta \gamma}} \mathbf{n}_{\beta \gamma} \cdot D_{A \beta} \nabla \tilde{c}_{A \beta} \mathrm{d} A-\frac{\epsilon_{\beta}^{-1}}{V} \int_{A_{\beta \omega}} \mathbf{n}_{\beta \omega} \cdot D_{A \beta} \nabla \tilde{c}_{A \beta} \mathrm{d} A \\
& -\frac{\epsilon_{\beta}^{-1}}{V} \int_{A_{\beta \sigma}} \mathbf{n}_{\beta \sigma} \cdot D_{A \beta} \nabla \tilde{c}_{A \beta} \mathrm{d} A
\end{aligned}
$$

$$
\begin{aligned}
& \text { B.C.1 }-\underbrace{\mathbf{n}_{\beta \sigma} \cdot D_{A \beta} \nabla\left\langle c_{A \beta}\right\rangle^{\beta}}_{\text {Source }}=\mathbf{n}_{\beta \sigma} \cdot D_{A \beta} \nabla \tilde{c}_{A \beta} \quad \text { at } A_{\beta \sigma} \\
& \text { B.C.2 }-\underbrace{\mathbf{n}_{\omega \sigma} \cdot \mathbf{D}_{\mathbf{A} \omega} K_{\beta \omega}^{-1} \cdot \nabla\left\langle c_{A \beta}\right\rangle^{\beta}}_{\text {Source }}=\mathbf{n}_{\omega \sigma} \cdot \mathbf{D}_{\mathbf{A} \omega} \cdot \nabla \tilde{c}_{A \omega} \quad \text { at } A_{\omega \sigma}
\end{aligned}
$$

B.C.3 $\tilde{c}_{A \beta}=K_{\beta \omega} \tilde{c}_{A \omega}$ at $A_{\beta \omega}$

$$
\begin{array}{cl}
\text { B.C.4 } & \underbrace{\mathbf{n}_{\beta \omega} \cdot\left(\left(\mathbf{v}_{\beta}-\mathbf{w}_{\beta \omega}\right)-K_{\beta \omega}^{-1}\left(\mathbf{v}_{\omega}-\mathbf{w}_{\omega \beta}\right)\right)\left\langle c_{A \beta}\right\rangle^{\beta}}_{\text {Source }} \\
& +\mathbf{n}_{\beta \omega} \cdot\left(\tilde{c}_{A \beta}\left(\mathbf{v}_{\beta}-\mathbf{w}_{\beta \omega}\right)-D_{A \beta} \nabla \tilde{c}_{A \beta}\right)
\end{array}
$$




$$
=\underbrace{\mathbf{n}_{\beta \omega} \cdot\left(D_{A \beta} \mathbf{I}-K_{\beta \omega}^{-1} D_{A \omega}\right) \cdot \nabla\left\langle c_{A \beta}\right\rangle^{\beta}}_{\text {Source }}+\mathbf{n}_{\beta \omega} \cdot\left(\tilde{c}_{A \omega}\left(\mathbf{v}_{\omega}-\mathbf{w}_{\omega \beta}\right)-\mathbf{D}_{\mathbf{A} \omega} \cdot \nabla \tilde{c}_{A \omega}\right) \text { at } A_{\beta \omega}
$$

B.C.5 $\tilde{c}_{A \omega}=\underbrace{K_{\beta \omega}^{-1}\left(c_{A \beta}^{\mathrm{eq}}-\left\langle c_{A \beta}\right\rangle^{\beta}\right)}_{\text {Source }}$ at $A_{\omega \gamma}$

B.C.6 $\tilde{c}_{A \beta}=\underbrace{c_{A \beta}^{\mathrm{eq}}-\left\langle c_{A \beta}\right\rangle^{\beta}}_{\text {Source }}$ at $A_{\beta \gamma}$

B.C.7(Periodicity) $\quad \tilde{c}_{A \beta}\left(\mathbf{r}+l_{i}\right)=\tilde{c}_{A \beta}(\mathbf{r}), \quad i=1,2,3$

B.C.8(Periodicity) $\tilde{c}_{A \omega}\left(\mathbf{r}+l_{i}\right)=\tilde{c}_{A \omega}(\mathbf{r}), \quad i=1,2,3$

B.C.9 $\left\langle\tilde{c}_{A \beta}\right\rangle^{\beta}=0$

B.C.10 $\left\langle\tilde{c}_{A \omega}\right\rangle^{\omega}=0$

$$
\begin{aligned}
\nabla .\left(\mathbf{D}_{\mathbf{A} \omega} \cdot \nabla \tilde{c}_{A \omega}\right)= & \frac{\epsilon_{\omega}^{-1}}{V} \int_{A_{\omega \gamma}} \mathbf{n}_{\omega \gamma} \cdot \tilde{c}_{A \omega}\left(\mathbf{v}_{\omega}-\mathbf{w}_{\omega \gamma}\right) \mathrm{d} A+\frac{\epsilon_{\omega}^{-1}}{V} \int_{A_{\omega \beta}} \mathbf{n}_{\omega \beta} \cdot \tilde{c}_{A \omega}\left(\mathbf{v}_{\omega}-\mathbf{w}_{\omega \beta}\right) \mathrm{d} A \\
& +\frac{\epsilon_{\omega}^{-1}}{V} \int_{A_{\omega \sigma}} \mathbf{n}_{\omega \sigma} \cdot \tilde{c}_{A \omega}\left(\mathbf{v}_{\omega}-\mathbf{w}_{\omega \sigma}\right) \mathrm{d} A-\frac{\epsilon_{\omega}^{-1}}{V} \int_{A_{\omega \gamma}} \mathbf{n}_{\omega \gamma} \cdot \mathbf{D}_{\mathbf{A} \omega} \cdot \nabla \tilde{c}_{A \omega} \mathrm{d} A \\
& -\frac{\epsilon_{\omega}^{-1}}{V} \int_{A_{\omega \beta}} \mathbf{n}_{\omega \beta} \cdot \mathbf{D}_{\mathbf{A} \omega} \cdot \nabla \tilde{c}_{A \omega} \mathrm{d} A-\frac{\epsilon_{\omega}^{-1}}{V} \int_{A_{\omega \sigma}} \mathbf{n}_{\omega \sigma} \cdot \mathbf{D}_{\mathbf{A} \omega} \cdot \nabla \tilde{c}_{A \omega} \mathrm{d} A
\end{aligned}
$$

\subsection{Closure Problems}

Following the developments in the literature (e.g., Quintard and Whitaker 1999), we have identified two non-homogeneous terms, $\nabla\left\langle c_{A \beta}\right\rangle^{\beta}$ and $\left(c_{A \beta}^{\mathrm{eq}}-\left\langle c_{A \beta}\right\rangle^{\beta}\right)$, that act as source terms for the deviation fields. We use these terms to formulate the closure problem solution. The mathematical structure of the coupled equations suggests, indeed, a solution of the form

$$
\begin{aligned}
& \tilde{c}_{A \beta}=\mathbf{b}_{\beta} \cdot \nabla\left\langle c_{A \beta}\right\rangle^{\beta}+s_{\beta}\left(c_{A \beta}^{\mathrm{eq}}-\left\langle c_{A \beta}\right\rangle^{\beta}\right) \\
& \tilde{c}_{A \omega}=\mathbf{b}_{\omega} \cdot K_{\beta \omega}^{-1} \nabla\left\langle c_{A \beta}\right\rangle^{\beta}+s_{\omega} K_{\beta \omega}^{-1}\left(c_{A \beta}^{\mathrm{eq}}-\left\langle c_{A \beta}\right\rangle^{\beta}\right)
\end{aligned}
$$

where the closure variables $\mathbf{b}_{\beta}, \mathbf{b}_{\omega}, s_{\beta}$ and $s_{\omega}$ satisfy the closure problems obtained by substituting Eqs. (78) and (79) into the deviations problems.

The two closure problems are specified below in their dimensionless form, and the full development of these problems is shown in "Appendix 2."

\section{Closure problems (dimensionless form) Problem I}

$$
\begin{aligned}
& \beta \text {-phase : } P e_{A}\left(\tilde{\mathbf{v}}_{\beta}^{\prime}+\mathbf{v}_{\beta}^{\prime} \cdot \nabla \mathbf{b}_{\beta}^{\prime}\right)=\nabla^{2} \mathbf{b}_{\beta}^{\prime}-\epsilon_{\beta}^{-1} \mathbf{U}_{\beta}^{\prime} \\
& \text { B.C.1 }-\mathbf{n}_{\beta \sigma} \cdot \nabla \mathbf{b}_{\beta}^{\prime}=\mathbf{n}_{\beta \sigma} \quad \text { at } A_{\beta \sigma} \\
& \text { B.C.2 }-\mathbf{n}_{\omega \sigma} \cdot \mathbf{D}_{\Gamma} \cdot \nabla \mathbf{b}_{\omega}^{\prime}=\mathbf{n}_{\omega \sigma} \cdot \mathbf{D}_{\Gamma} \text { at } A_{\omega \sigma} \\
& \text { B.C.3 } \mathbf{b}_{\beta}^{\prime}=\mathbf{b}_{\omega}^{\prime} \text { at } A_{\beta \omega} \\
& \text { B.C.4 }-\mathbf{n}_{\beta \omega} \cdot \mathbf{D}_{\Gamma} \cdot \nabla \mathbf{b}_{\omega}^{\prime}=-\mathbf{n}_{\beta \omega} \cdot\left(\mathbf{I}-\mathbf{D}_{\Gamma}\right)-\mathbf{n}_{\beta \omega} . \nabla \mathbf{b}_{\beta}^{\prime} \text { at } A_{\beta \omega}
\end{aligned}
$$




$$
\begin{aligned}
& \text { B.C.5 } \mathbf{b}_{\beta}^{\prime}=0 \text { at } A_{\beta \gamma} \\
& \text { B.C.6 } \mathbf{b}_{\omega}^{\prime}=0 \text { at } A_{\omega \gamma} \\
& \text { B.C.7(Periodicity) } \quad \mathbf{b}_{\beta}^{\prime}\left(\mathbf{r}+l_{i}\right)=\mathbf{b}_{\beta}^{\prime}(\mathbf{r}), i=1,2,3 \quad i=1,2,3 \\
& \text { B.C.8(Periodicity) } \mathbf{b}_{\omega}^{\prime}\left(\mathbf{r}+l_{i}\right)=\mathbf{b}_{\omega}^{\prime}(\mathbf{r}), i=1,2,3 \quad i=1,2,3 \\
& \text { B.C.9 }\left\langle\mathbf{b}_{\beta}^{\prime}\right\rangle^{\beta}=0 \\
& \text { B.C.10 }\left\langle\mathbf{b}_{\omega}^{\prime}\right\rangle^{\omega}=0 \\
& \omega \text {-phase : } \mathbf{D}_{\boldsymbol{\Gamma}} \cdot \nabla^{2} \mathbf{b}_{\omega}^{\prime}=\mathbf{D}_{\Gamma} \epsilon_{\omega}^{-1} \mathbf{U}_{\omega}^{\prime} \\
& \mathbf{U}_{\beta}^{\prime}=\frac{1}{V} \int_{A_{\beta \gamma}} \mathbf{n}_{\beta \gamma} \cdot \nabla \mathbf{b}_{\beta}^{\prime} \mathrm{d} A+\frac{1}{V} \int_{A_{\beta \omega}} \mathbf{n}_{\beta \omega} \cdot \nabla \mathbf{b}_{\beta}^{\prime} \mathrm{d} A \\
& +\frac{1}{V} \int_{A_{\beta \sigma}} \mathbf{n}_{\beta \sigma} \cdot \nabla \mathbf{b}_{\beta}^{\prime} \mathrm{d} A \\
& \mathbf{U}_{\omega}^{\prime}=\frac{1}{V} \int_{A_{\omega \gamma}} \mathbf{n}_{\omega \gamma} \cdot \nabla \mathbf{b}_{\omega}^{\prime} \mathrm{d} A+\frac{1}{V} \int_{A_{\omega \beta}} \mathbf{n}_{\omega \beta} \cdot \nabla \mathbf{b}_{\omega}^{\prime} \mathrm{d} A \\
& +\frac{1}{V} \int_{A_{\omega \sigma}} \mathbf{n}_{\omega \sigma} \cdot \nabla \mathbf{b}_{\omega}^{\prime} \mathrm{d} A
\end{aligned}
$$

\section{Problem II}

$$
\begin{aligned}
& \beta \text {-phase : } P e_{A} \mathbf{v}_{\beta}^{\prime} \cdot \nabla s_{\beta^{\prime}}=\nabla^{2} s_{\beta^{\prime}}-\epsilon_{\beta}^{-1} \mathbf{S}_{\beta}^{\prime} \\
& \text { B.C.1 }-\mathbf{n}_{\beta \sigma} \cdot \nabla s_{\beta}^{\prime}=0 \text { at } A_{\beta \sigma} \\
& \text { B.C.2 }-\mathbf{n}_{\omega \sigma} \cdot \mathbf{D}_{\Gamma} \cdot \nabla s_{\omega}^{\prime}=0 \text { at } A_{\omega \sigma} \\
& \text { B.C.3 } s_{\beta}^{\prime}=s_{\omega}^{\prime} \text { at } A_{\beta \omega} \\
& \text { B.C.4 }-\mathbf{n}_{\beta \omega} \cdot \nabla s_{\beta}^{\prime}=-\mathbf{n}_{\beta \omega} \cdot \mathbf{D}_{\Gamma} \cdot \nabla s_{\omega}^{\prime} \text { at } A_{\beta \omega} \\
& \text { B.C.5 } s_{\beta}^{\prime}=1 \text { at } A_{\beta \gamma} \\
& \text { B.C.6 } s_{\omega}^{\prime}=1 \text { at } A_{\omega \gamma} \\
& \text { B.C.7 (Periodicity) } s_{\beta}^{\prime}\left(\mathbf{r}+l_{i}\right)=s_{\beta}^{\prime}(r), i=1,2,3 \quad i=1,2,3 \\
& \text { B.C.8 (Periodicity) } s_{\omega}^{\prime}\left(\mathbf{r}+l_{i}\right)=s_{\omega}^{\prime}(r), i=1,2,3 \quad i=1,2,3 \\
& \text { B.C. } 9\left\langle s_{\beta}^{\prime}\right\rangle^{\beta}=0 \\
& \text { B.C.10 }\left\langle s_{\omega}^{\prime}\right\rangle^{\omega}=0 \\
& \mathbf{S}_{\beta}^{\prime}=\frac{1}{V} \int_{A_{\beta \gamma}} \mathbf{n}_{\beta \gamma} . \nabla s_{\beta}^{\prime} \mathrm{d} A+\frac{1}{V} \int_{A_{\beta \omega}} \mathbf{n}_{\beta \omega} . \nabla s_{\beta}^{\prime} \mathrm{d} A \\
& +\frac{1}{V} \int_{A_{\beta \sigma}} \mathbf{n}_{\beta \sigma} \cdot \nabla s_{\beta}^{\prime} \mathrm{d} A
\end{aligned}
$$




$$
\begin{aligned}
\mathbf{S}_{\omega}^{\prime}= & \frac{1}{V} \int_{A_{\omega \gamma}} \mathbf{n}_{\omega \gamma} \cdot \nabla s_{\omega}^{\prime} \mathrm{d} A+\frac{1}{V} \int_{A_{\omega \beta}} \mathbf{n}_{\omega \beta} \cdot \nabla s_{\omega}^{\prime} \mathrm{d} A \\
& +\frac{1}{V} \int_{A_{\omega \sigma}} \mathbf{n}_{\omega \sigma} \cdot \nabla s_{\omega}^{\prime} \mathrm{d} A
\end{aligned}
$$

where the dimensionless variables and the parameters have been defined by

$$
\begin{gathered}
\tilde{\mathbf{v}}_{\beta}^{\prime}=\frac{\tilde{\mathbf{v}}_{\beta}}{\left\|\mathbf{v}_{\beta}\right\|} \quad \mathbf{v}_{\beta}^{\prime}=\frac{\mathbf{v}_{\beta}}{\left\|\mathbf{v}_{\beta}\right\|} \\
\mathbf{D}_{\boldsymbol{\Gamma}}=\frac{K_{\beta \omega}^{-1} \mathbf{D}_{\mathbf{A} \omega}}{D_{A \beta}} \quad P e_{A}=\frac{\left\|\mathbf{v}_{\beta}\right\| l_{\beta}}{D_{A \beta}}
\end{gathered}
$$

\section{Closed Form of the Macroscopic Equation}

Now, injecting the closure solutions (Eqs. (78), (79)) into the unclosed averaged equation, the following closed form of the $\beta$-phase transport equation is obtained

$$
\begin{gathered}
\underbrace{\frac{\partial}{\partial t}\left(\epsilon_{\beta}+\epsilon_{\omega} K_{\beta \omega}^{-1}\right)\left\langle c_{A \beta}\right\rangle^{\beta}}_{\text {Accumulation }}+\underbrace{\epsilon_{\beta}\left\langle\mathbf{v}_{\beta}\right\rangle^{\beta} \cdot \nabla\left\langle c_{A \beta}\right\rangle^{\beta}}_{\text {Advection }}+\underbrace{\nabla \cdot\left[\mathbf{d}_{\beta \omega}\left(c_{A \beta}^{\mathrm{eq}}-\left\langle c_{A \beta}\right\rangle^{\beta}\right)\right]}_{\text {Advection correction }} \\
-\underbrace{\mathbf{U}_{\beta \omega} \cdot \nabla\left\langle c_{A \beta}\right\rangle^{\beta}}_{\text {Advection correction }} \\
=\underbrace{\nabla \cdot\left(\mathbf{D}_{\beta \omega}^{*} \cdot \nabla\left\langle c_{A \beta}\right\rangle^{\beta}\right)}_{\text {Dispersion }}+\underbrace{\alpha_{A}^{*}\left(c_{A \beta}^{\mathrm{eq}}-\left\langle c_{A \beta}\right\rangle^{\beta}\right)}_{\text {Mass exchange }} \\
-\underbrace{\epsilon_{\omega} \mu_{A \omega} \rho_{\omega} c_{B \omega}^{*}-c_{\text {Reaction }}}_{\left\langle c_{A \beta}\right\rangle^{\beta}}
\end{gathered}
$$

and one can use the representation for the spatial deviation concentrations given by Eqs. (78) and (79) to express the macroscopic form of the $\gamma$-phase equation

$$
\begin{aligned}
& \rho_{\gamma} \frac{\partial \epsilon_{\gamma}}{\partial t}+\left(\frac{1}{V} \int_{A_{\beta \gamma}} \mathbf{n}_{\beta \gamma} \cdot D_{A \beta}\left(\mathbf{I}+\nabla \mathbf{b}_{\beta}\right) \mathrm{d} A+\frac{K_{\beta \omega}^{-1}}{V} \int_{A_{\omega \gamma}} \mathbf{n}_{\omega \gamma} \cdot \mathbf{D}_{\mathbf{A} \omega} \cdot\left(\mathbf{I}+\nabla \mathbf{b}_{\omega}\right) \mathrm{d} A\right) . \\
& \quad \nabla\left\langle c_{A \beta}\right\rangle^{\beta} \\
& =-\alpha_{A}^{*}\left(c_{A \beta}^{\mathrm{eq}}-\left\langle c_{A \beta}\right\rangle^{\beta}\right)
\end{aligned}
$$

We arrange the diffusive term of this equation (Eq. (111)) by using the following relationship (additional details are available in Quintard and Whitaker (1999))

$$
\begin{gathered}
\frac{1}{V} \int_{A_{\beta \gamma}} \mathbf{n}_{\beta \gamma} \cdot D_{A \beta}\left(\mathbf{I}+\nabla \mathbf{b}_{\beta}\right) \mathrm{d} A=-D_{A \beta} \nabla \epsilon_{\beta} \\
\frac{1}{V} \int_{A_{\omega \gamma}} \mathbf{n}_{\omega \gamma} \cdot \mathbf{D}_{\mathbf{A} \omega} \cdot\left(\mathbf{I}+\nabla \mathbf{b}_{\omega}\right) \mathrm{d} A=-\mathbf{D}_{\mathbf{A} \omega} \cdot \nabla \epsilon_{\omega}
\end{gathered}
$$


Thus, Eq. (111) becomes

$$
\rho_{\gamma} \frac{\partial \epsilon_{\gamma}}{\partial t}=\left(\nabla \epsilon_{\beta}+\mathbf{D}_{\Gamma} \cdot \nabla \epsilon_{\omega}\right) \cdot D_{A \beta} \nabla\left\langle c_{A \beta}\right\rangle^{\beta}-\alpha_{A}^{*}\left(c_{A \beta}^{\mathrm{eq}}-\left\langle c_{A \beta}\right\rangle^{\beta}\right)
$$

A reasonable estimate of the gradient of the average concentration in the case of purely convective transport is given by Quintard and Whitaker (1999)

$$
\nabla\left\langle c_{A \beta}\right\rangle^{\beta}=\mathcal{O}\left[\frac{1}{\left\langle\mathbf{v}_{\beta}\right\rangle} \alpha_{A}^{*}\left(c_{A \beta}^{\mathrm{eq}}-\left\langle c_{A \beta}\right\rangle^{\beta}\right)\right],
$$

and Eq. (115) leads to

$$
\left(\nabla \epsilon_{\beta}+\mathbf{D}_{\Gamma} \cdot \nabla \epsilon_{\omega}\right) \cdot D_{A \beta} \nabla\left\langle c_{A \beta}\right\rangle^{\beta}=\mathcal{O}\left[\frac{l_{\beta}\left(\nabla \epsilon_{\beta}+\mathbf{D}_{\Gamma} \cdot \nabla \epsilon_{\omega}\right)}{P e_{A}} \alpha_{A}^{*}\left(c_{A \beta}^{\mathrm{eq}}-\left\langle c_{A \beta}\right\rangle^{\beta}\right)\right]
$$

Under this constraint (Eq. (116)), it is clear that the additional coupling term $\left(\nabla \epsilon_{\beta}+\right.$ $\left.\mathbf{D}_{\Gamma} \cdot \nabla \epsilon_{\omega}\right) . D_{A \beta} \nabla\left\langle c_{A \beta}\right\rangle^{\beta}$ can be neglected

$$
\left(\nabla \epsilon_{\beta}+\mathbf{D}_{\Gamma} \cdot \nabla \epsilon_{\omega}\right) \cdot D_{A \beta} \nabla\left\langle c_{A \beta}\right\rangle^{\beta}<<\alpha_{A}^{*}\left(c_{A \beta}^{\mathrm{eq}}-\left\langle c_{A \beta}\right\rangle^{\beta}\right)
$$

and the classical form in the literature for the mass balance equation of the NAPL phase $(\gamma$-phase $)$ is recovered

$$
\rho_{\gamma} \frac{\partial \epsilon_{\gamma}}{\partial t}=-\alpha_{A}^{*}\left(c_{A \beta}^{\mathrm{eq}}-\left\langle c_{A \beta}\right\rangle^{\beta}\right)
$$

where $\mathbf{D}_{\beta \omega}^{*}$ (Darcy-scale effective diffusion tensor for the species $A$ ), $\mathbf{d}_{\beta \omega}$ (a velocity-like effective transport coefficient), $\alpha_{A}^{*}$ (mass transfer coefficient for the species $A$ ), $\mathbf{U}_{\beta \omega}$ (a velocity-like effective transport coefficient) are given by

$$
\begin{array}{r}
\mathbf{D}_{\beta \omega}^{*}=\left(\epsilon_{\beta} D_{A \beta} \mathbf{I}+K_{\beta \omega}^{-1} \epsilon_{\omega} \mathbf{D}_{\mathbf{A} \omega}\right)+D_{A \beta} \mathbf{m}_{\beta}+K_{\beta \omega}^{-1} \mathbf{D}_{\mathbf{A} \omega} \cdot \mathbf{m}_{\omega}-\left\langle\tilde{\mathbf{v}}_{\beta} b_{\beta}\right\rangle \\
\mathbf{d}_{\beta \omega}=\left\langle\tilde{\mathbf{v}}_{\beta} s_{\beta}\right\rangle-D_{A \beta} \mathbf{l}_{\beta}-K_{\beta \omega}^{-1} \mathbf{D}_{\mathbf{A} \omega} \cdot \mathbf{l}_{\omega} \\
\alpha_{A}^{*}=\frac{D_{A \beta}}{V} \int_{A_{\beta \gamma}} \mathbf{n}_{\beta \gamma} \nabla s_{\beta} \mathrm{d} A+K_{\beta \omega}^{-1} \mathbf{D}_{\mathbf{A} \omega} \cdot\left(\frac{1}{V} \int_{A_{\omega \gamma}} \mathbf{n}_{\omega \gamma} \nabla s_{\omega} \mathrm{d} A\right) \\
\mathbf{U}_{\beta \omega}=\frac{D_{A \beta}}{V} \int_{A_{\beta \gamma}} \mathbf{n}_{\beta \gamma} \nabla \mathbf{b}_{\beta} \mathrm{d} A+K_{\beta \omega}^{-1} \mathbf{D}_{\mathbf{A} \omega} \cdot\left(\frac{1}{V} \int_{A_{\omega \gamma}} \mathbf{n}_{\omega \gamma} \nabla \mathbf{b}_{\omega} \mathrm{d} A\right)
\end{array}
$$

where

$$
\begin{aligned}
& \mathbf{m}_{\beta}=\frac{1}{V} \int_{A_{\beta \gamma}} \mathbf{n}_{\beta \gamma} \mathbf{b}_{\beta} \mathrm{d} A+\frac{1}{V} \int_{A_{\beta \omega}} \mathbf{n}_{\beta \omega} \mathbf{b}_{\beta} \mathrm{d} A+\frac{1}{V} \int_{A_{\beta \sigma}} \mathbf{n}_{\beta \sigma} \mathbf{b}_{\beta} \mathrm{d} A \\
& \mathbf{m}_{\omega}=\frac{1}{V} \int_{A_{\omega \gamma}} \mathbf{n}_{\omega \gamma} \mathbf{b}_{\omega} \mathrm{d} A+\frac{1}{V} \int_{A_{\omega \beta}} \mathbf{n}_{\omega \beta} \mathbf{b}_{\omega} \mathrm{d} A+\frac{1}{V} \int_{A_{\omega \sigma}} \mathbf{n}_{\omega \sigma} \mathbf{b}_{\omega} \mathrm{d} A \\
& \mathbf{l}_{\beta}=\frac{1}{V} \int_{A_{\beta \gamma}} \mathbf{n}_{\beta \gamma} \mathbf{s}_{\beta} \mathrm{d} A+\frac{1}{V} \int_{A_{\beta \omega}} \mathbf{n}_{\beta \omega} \mathbf{s}_{\beta} \mathrm{d} A+\frac{1}{V} \int_{A_{\beta \sigma}} \mathbf{n}_{\beta \sigma} \mathbf{s}_{\beta} \mathrm{d} A \\
& \mathbf{l}_{\omega}=\frac{1}{V} \int_{A_{\omega \gamma}} \mathbf{n}_{\omega \gamma} \mathbf{s}_{\omega} \mathrm{d} A+\frac{1}{V} \int_{A_{\omega \beta}} \mathbf{n}_{\omega \beta} \mathbf{s}_{\omega} \mathrm{d} A+\frac{1}{V} \int_{A_{\omega \sigma}} \mathbf{n}_{\omega \sigma} \mathbf{s}_{\omega} \mathrm{d} A
\end{aligned}
$$


Note that the dimensionless form of the mass exchange coefficient and the dispersion tensor, used hereafter, are defined by

$$
\begin{gathered}
\alpha_{A}^{*^{\prime}}=\frac{1}{V} \int_{A_{\beta \gamma}} \mathbf{n}_{\beta \gamma} \nabla s_{\beta}^{\prime} \mathrm{d} A+\mathbf{D}_{\Gamma} \cdot\left(\frac{1}{V} \int_{A_{\omega \gamma}} \mathbf{n}_{\omega \gamma} \nabla s_{\omega}^{\prime} \mathrm{d} A\right) \\
\mathbf{D}_{\beta \omega}^{*^{\prime}}=\left(\epsilon_{\beta} \mathbf{I}+\epsilon_{\omega} \mathbf{D}_{\Gamma}\right)+\mathbf{m}_{\beta}^{\prime}+\mathbf{D}_{\Gamma} \cdot \mathbf{m}_{\omega}^{\prime}-P e_{A}\left\langle\tilde{\mathbf{v}}_{\beta}^{\prime} \mathbf{b}_{\beta}^{\prime}\right\rangle
\end{gathered}
$$

where

$$
\begin{aligned}
& \mathbf{m}_{\beta}^{\prime}=\frac{1}{V} \int_{A_{\beta \gamma}} \mathbf{n}_{\beta \gamma} \mathbf{b}_{\beta}^{\prime} \mathrm{d} A+\frac{1}{V} \int_{A_{\beta \omega}} \mathbf{n}_{\beta \omega} \mathbf{b}_{\beta}^{\prime} \mathrm{d} A+\frac{1}{V} \int_{A_{\beta \sigma}} \mathbf{n}_{\beta \sigma} \mathbf{b}_{\beta}^{\prime} \mathrm{d} A \\
& \mathbf{m}_{\omega}^{\prime}=\frac{1}{V} \int_{A_{\omega \gamma}} \mathbf{n}_{\omega \gamma} \mathbf{b}_{\omega}^{\prime} \mathrm{d} A+\frac{1}{V} \int_{A_{\omega \beta}} \mathbf{n}_{\omega \beta} \mathbf{b}_{\omega}^{\prime} \mathrm{d} A+\frac{1}{V} \int_{A_{\omega \sigma}} \mathbf{n}_{\omega \sigma} \mathbf{b}_{\omega}^{\prime} \mathrm{d} A
\end{aligned}
$$

\section{Results and Discussion}

The averaging procedure leads to the closed form of the macroscale equations given by Eqs. (110) and (111) and two closure problems. The closure problems need to be solved on a representative unit cell of the pore scale geometry to deduce with accuracy the effective properties of the porous medium. Ideally, the choice of unit cells should be based on the results of direct experimental measurements conducted at both the microscale and macroscale. Theoretically, it is possible to obtain a three-dimensional image of the four phases, biofilm-fluid-NAPLsolid, and this is an area of active research (Davit et al. 2010; Seymour et al. 2004). However, the results from such multiscale experimental measurements are currently not available. Obviously, the 2D unit cells, illustrated in Fig. 3, may not capture all the features of a real system; however, they are sufficient to gain a significant insight about the physical mechanisms involved. Additionally, they could be used easily in conjunction with micromodel studies (Bahar et al. 2015). In this section, we describe the calculation steps of two effective parameters that are the dispersion tensor, Eq. (119), and the mass exchange coefficient, Eq. (121). Such calculations give us an overview of how the pore scale architecture (especially biofilm and NAPL distribution) impacts these macroscale coefficients and illustrates the capability of the model. A large literature is available on these two parameters which justifies our choice (Quintard and Whitaker 1994; Ahmadi et al. 2001; Golfier et al. 2009). Therefore, different types of complex unit cells have been used, they are summarized in Fig. 3. Most important features that characterize unit cells are gathered in Table 1.

\subsection{Results for Complex Unit Cells}

As specified above, the calculation of the dispersion tensor and the mass exchange coefficient are based on the different two-dimensional unit cells represented in Fig. 3 and are denoted from (a) to (e). Their main morphological features (e.g., circularity, interfacial area, Feret diameter) and flow properties (permeability, wetting phase) are gathered in Table 1. These unit cells were constructed from a tomographic image of sandstone for the solid phase. We insist on the fact that these geometries do not pretend to give a fair picture of a real rock sample. Their only purpose is to be complex enough to capture the main features (tortuosity effect, different pore sizes, and different NAPL blobs or biofilm shapes) of a contaminated soil. The volume fraction of the $\sigma$-phase is kept constant in all configurations and fixed at 0.5 . The pore throat characteristic length, $l_{\beta}$, is estimated to be $0.3 \mathrm{~mm}$ so that the constraint 

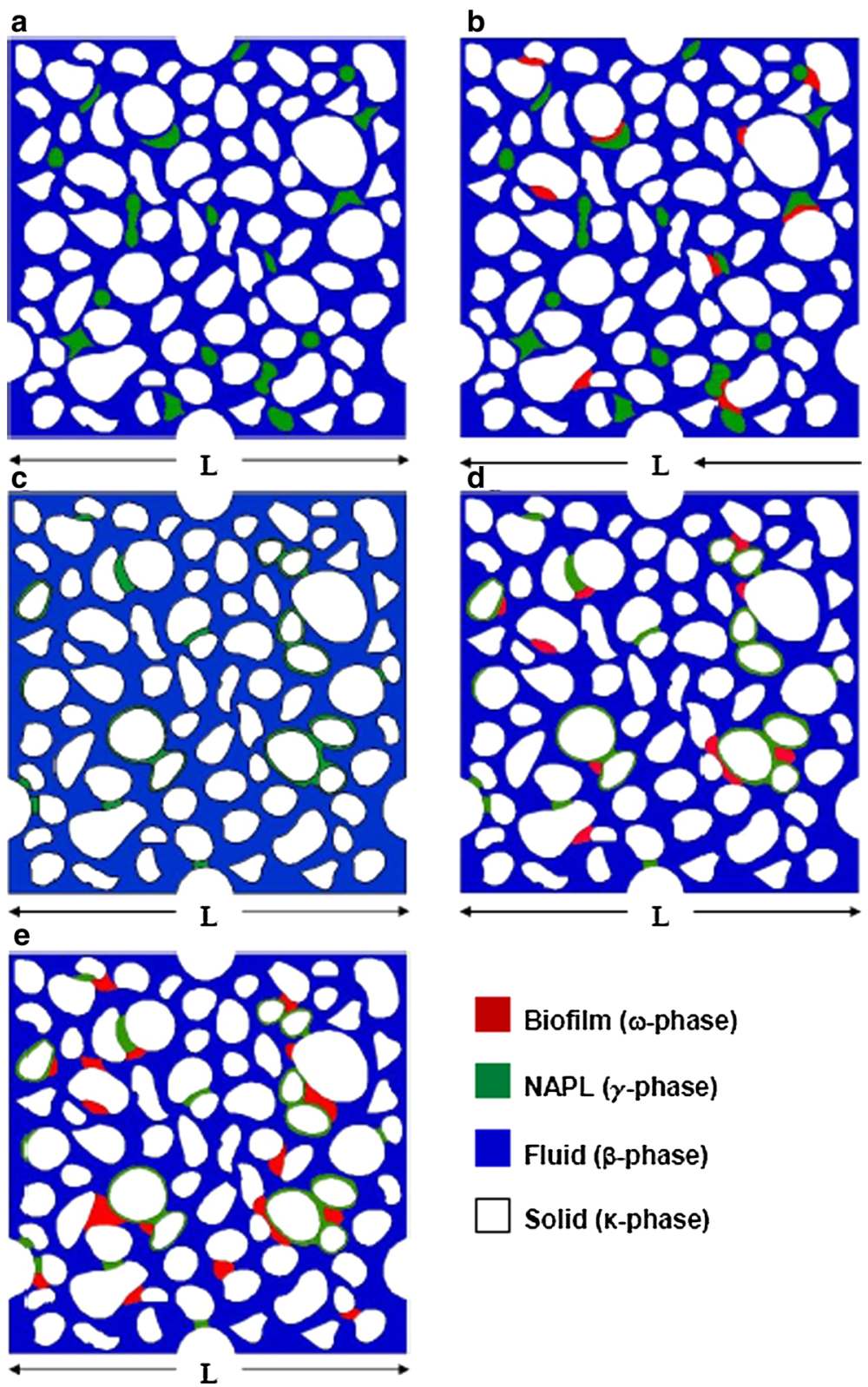

I

Biofilm ( $\omega$-phase)

NAPL $(\gamma$-phase $)$

Fluid ( $\beta$-phase)

Solid (K-phase)

Fig. 3 Unit cells used for computations: non-wetting cases (unit cell $\mathbf{a}$ : without biofilm—unit cell $\mathbf{b}$ : after bacteria colonization) and wetting cases (unit cell $\mathbf{c}$ : without biofilm—unit cells (d) and (e): after bacteria colonization)

of separation of scales is verified. All the unit cells (as shown in Fig. 3) have the same size, i.e., $L \times L=12 \times 12 \mathrm{~mm}^{2}$. Biofilm phase in the unit cells (b), (d), and (e) was generated arbitrarily during their construction, and the values concerning the volume fractions are summarized in Table 1. The NAPL blob distribution in the different unit cells was also done randomly by respecting the wettability of the NAPL phase. The resulting porous network of 
Table 1 Physical parameters associated with the various unit cells

\begin{tabular}{|c|c|c|c|c|c|}
\hline \multirow[t]{2}{*}{ Parameter } & \multicolumn{5}{|l|}{ Unit cell } \\
\hline & (a) & (b) & (c) & (d) & (e) \\
\hline Fluid volume fraction $\epsilon_{\beta}$ & 0.469 & 0.46 & 0.471 & 0.47 & 0.44 \\
\hline $\begin{array}{l}\text { Biofilm volume fraction } \\
\epsilon_{\omega}\end{array}$ & - & 0.011 & - & 0.011 & 0.028 \\
\hline NAPL volume fraction $\epsilon_{\gamma}$ & 0.032 & 0.032 & 0.030 & 0.030 & 0.030 \\
\hline Permeability $k\left(\mathrm{~m}^{2}\right)$ & $2.47 \times 10^{-14}$ & $2.21 \times 10^{-14}$ & $2.48 \times 10^{-14}$ & $2.36 \times 10^{-14}$ & $2.17 \times 10^{-14}$ \\
\hline $\begin{array}{l}\beta-\gamma \text { Interfacial area } A_{v} \\
\left(\mathrm{~mm}^{-1}\right)\end{array}$ & 0.005 & 0.0038 & 0.0066 & 0.0059 & 0.0049 \\
\hline $\begin{array}{l}\text { Feret diameter } D_{f}(\omega \text { - } \\
\text { and } \gamma \text {-phases })(\mathrm{mm})\end{array}$ & 0.16 & 0.12 & 0.16 & 0.15 & 0.13 \\
\hline $\begin{array}{l}\text { Circularity } C_{f}(\omega \text { - and } \\
\gamma \text {-phases })\end{array}$ & 0.86 & 0.86 & 0.85 & 0.87 & 0.85 \\
\hline $\begin{array}{l}\text { Lacunarity } \lambda_{f}(\omega \text { - and } \\
\gamma \text {-phases })\end{array}$ & 0.47 & 0.50 & 0.37 & 0.43 & 0.50 \\
\hline Wetting phase & Water & Water & NAPL & NAPL & NAPL \\
\hline
\end{tabular}

these complex unit cells contains ganglia of different sizes and forms similar to real-world conditions (insular saturation for non-wetting NAPL; films or pendular rings when oil is wetting). Note that the geometrical properties of NAPL blobs and biofilms clusters in the different unit cells are globally similar in terms of circularity and characteristic length (Feret diameter), but the heterogeneity of phase distribution (i.e., lacunarity) varies. Moreover, the change in wettability obviously affects the interfacial area values which drive mass transfer processes, as detailed in Table 1 .

The first step in our approach consists in calculating the pore scale velocity field from a given inlet velocity or pressure gradient. The velocity field is obtained by solving Stokes equations, with symmetry conditions on lateral boundaries, over the entire system. All numerical calculations were performed using COMSOL Multiphysics Sofware ${ }^{\circledR}$ based on the finite element formulation. We used quadratic Lagrange elements for the velocities and linear for the pressure to obtain the numerical solution. The resulting linear system was solved with the direct solver PARDISO (Schenk and Gärtner 2004) based on the LU decomposition method.

In the second step, we solve the closure problems that are problems I and II for computing, respectively, the effective dispersion tensor and the mass exchange coefficient. These effective properties are estimated for different arbitrary values of the Péclet number varying between $10^{-3}$ and $10^{3}$. For each computation, the solution convergence analysis was carried out. This analysis confirmed the independence of the numerical results with respect to the grid resolution.

\subsection{Impact of the NAPL Wettability Configuration (a) and (c)}

First, we examined the problem of dissolution of a NAPL phase without biofilm (abiotic conditions) represented by the unit cells (a) and (c). Our objective was to test the impact of the NAPL wettability on the dispersion coefficient ( $x x$-component and $y y$-component of the dispersion tensor) and the mass exchange coefficient. Several studies have shown the influence of spatial variations in wettability on NAPL dissolution (Bradford et al. 2000; Seyedabbasi et al. 2008). In particular, we compare these effective properties in the NAPL-wetting case 


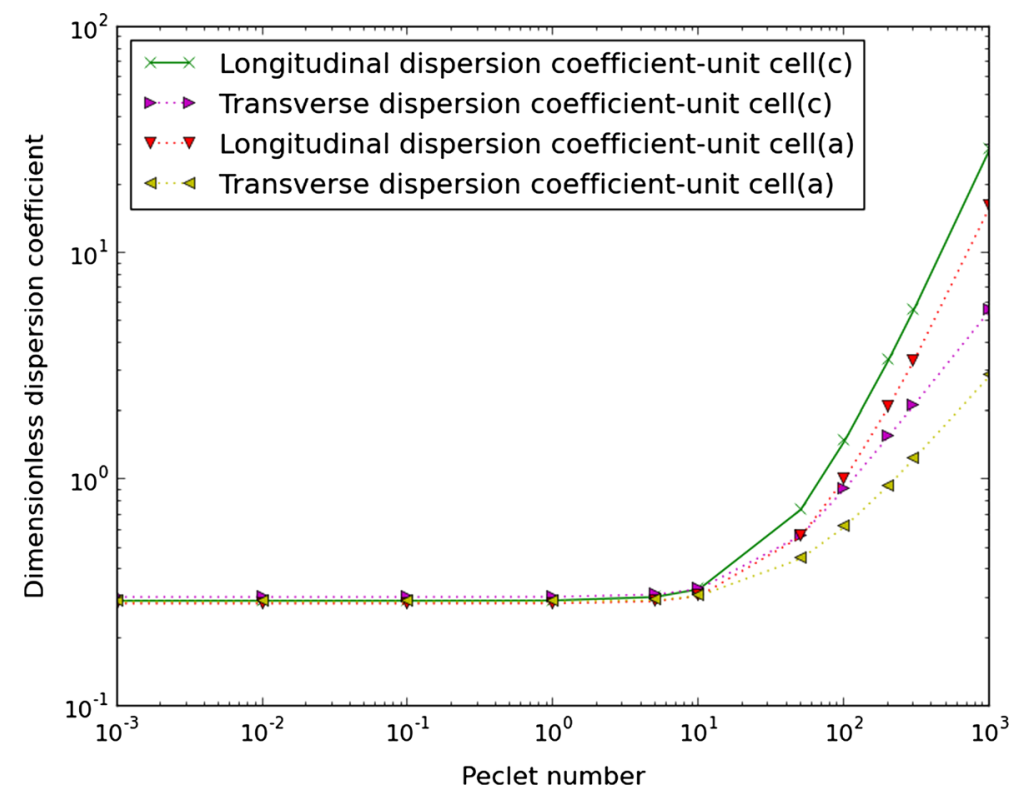

Fig. 4 Dimensionless dispersion coefficient: variation versus $P e_{A}$ in abiotic conditions

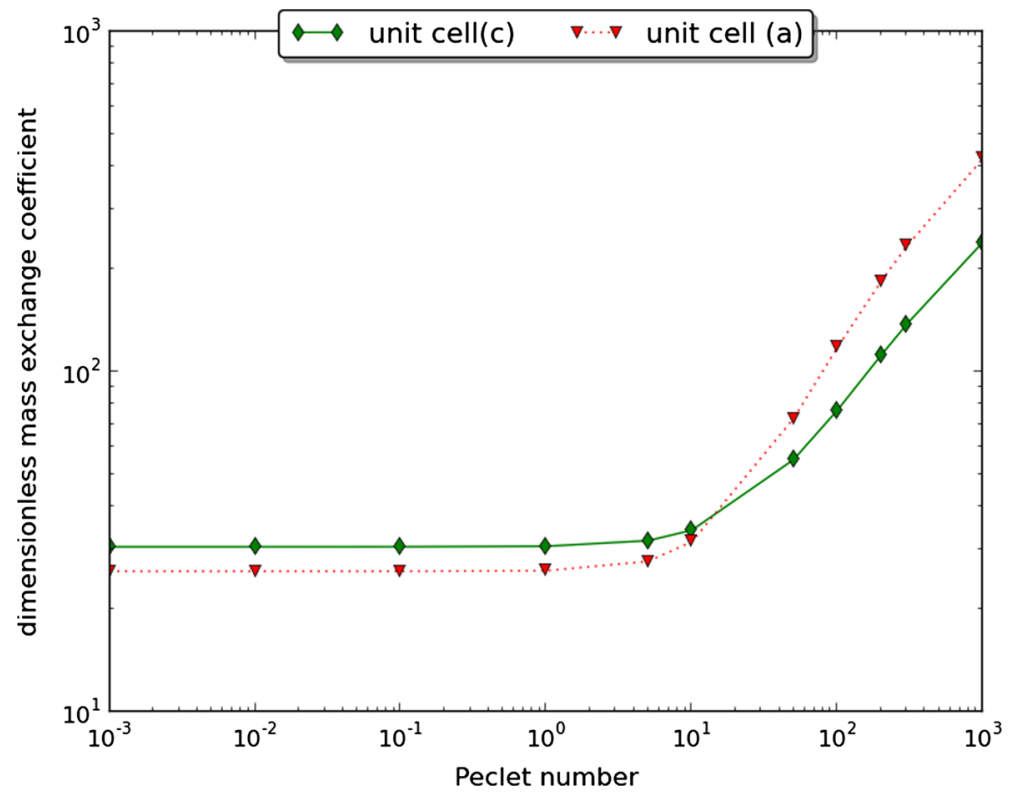

Fig. 5 Dimensionless mass exchange coefficient: variation versus $P e_{A}$ in abiotic conditions

(unit cell (c) -Fig. 3) and the non-wetting case (unit cell (a)-Fig. 3). The results for the longitudinal and transverse dispersion coefficients are shown in Fig. 4 and those for the mass exchange coefficient are represented in Fig. 5. Results for both cases illustrate the typical behavior of these effective properties with respect to the Péclet number. 
Velocity field of wetting case

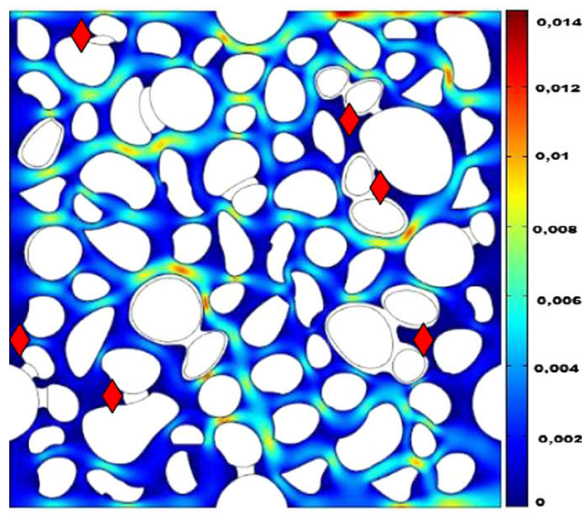

$\checkmark$ Dead-flow zones
Velocity field of non-wetting case

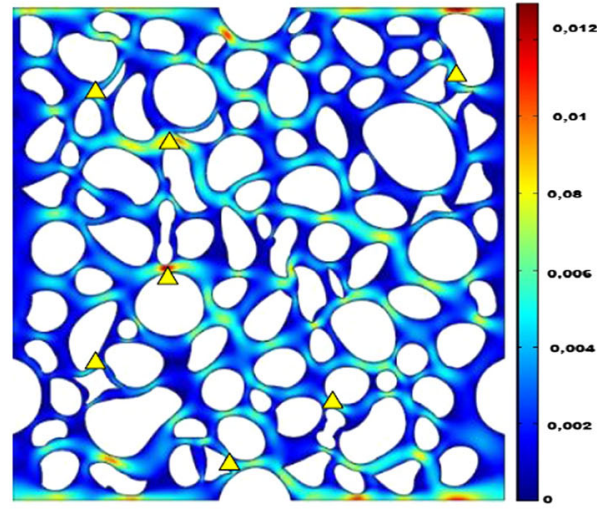

$\triangle \quad$ Higher velocities zones

Fig. 6 Velocity field: Comparison of the unit cells without biofilm (unit cell (c) and (a))

In the diffusive regime, i.e., at low Péclet number, as the dispersion coefficient is essentially determined by the volume fraction of each phase, we recover the same value of the effective diffusion, whatever the case. On the contrary, the two geometries exhibit a different behavior for the dimensionless mass exchange coefficient (Fig. 6). Mass transfer, indeed, is mainly driven by the surface exchange area $A_{\beta \gamma}$ which vary significantly between the unit cells (a) and (c) due to the presence of NAPL rings around the solid phase in the oil-wetting case.

As soon as the advection term becomes predominant, the difference between the two longitudinal dispersion curves increases (Fig. 4) leading, for a large Péclet $\left(P e_{A}=10^{3}\right)$, to a longitudinal dispersion coefficient value for the unit cell (c) about two times larger than that obtained for the unit cell (a). This discrepancy can be explained by the configuration of the unit cell (a) marked by isolated NAPL blobs. These blobs increase the mechanical effects of the dispersion which is not anymore necessarily oriented in the flow direction. We note that our results are similar with those obtained by Quintard and Whitaker (1994) for active dispersion in the presence of NAPL. Concerning the dimensionless exchange coefficient, the trend observed at low $P e_{A}$ is inverted, and we observe a coefficient value smaller in the wetting case (unit cell (c)) than for the non-wetting case (unit cell (a)). To support these results, we have represented in Fig. 6 the spatial distribution of the velocity fields obtained for both geometries. The analysis of this figure indicates that the velocity magnitude in the vicinity of the fluid-NAPL interface is globally higher for the non-wetting case (zones represented by yellow triangles in Fig. 6), and we note the presence of dead-flow zones close to the NAPL blobs for the unit cell (c) (zones represented by red diamonds in Fig. 6). These higher local values of velocity mechanically contribute to increase the mass transfer at the macroscale as has been shown by Miller and Poirier-Mcnell (1990).

\subsection{Impact of the Biofilm Development: Comparison of Wetting Cases (Before and After Colonization by Biofilm)_Configurations (c), (d), and (e)}

The results reported in Figs. 7 and 8 illustrate the impact of the biofilm growth on the dispersion coefficient and mass transfer coefficient in the oil-wetting case (unit cell (c): 


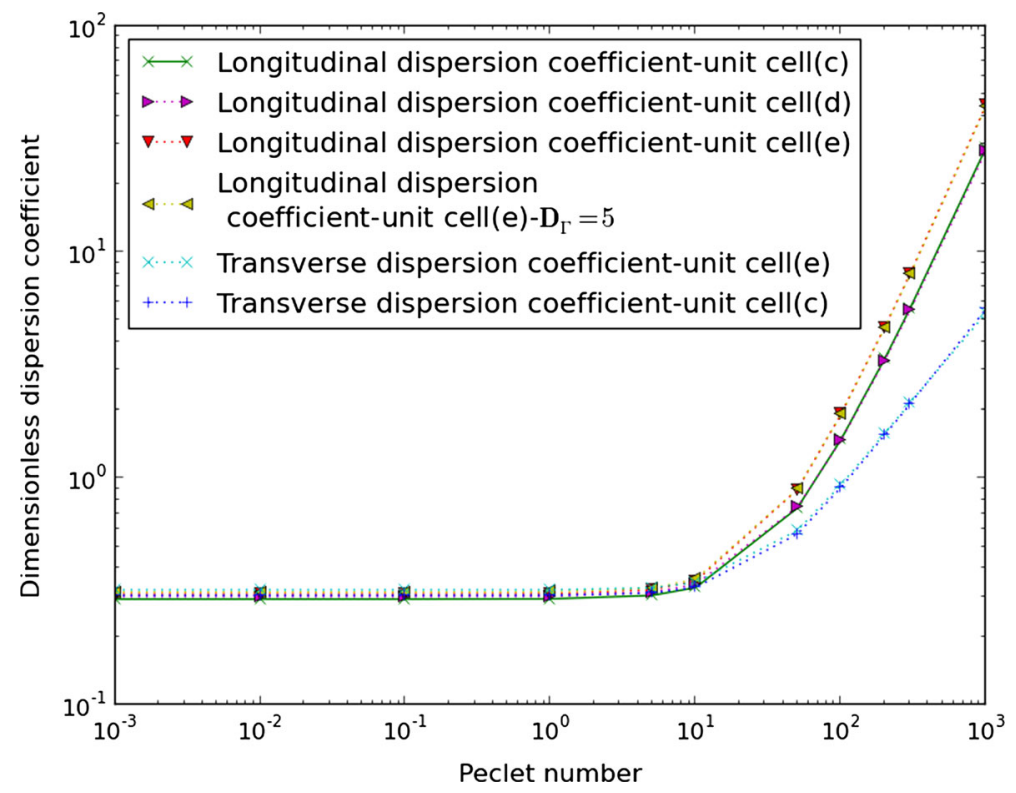

Fig. 7 Dimensionless dispersion coefficient: comparison of NAPL-wetting cases (before and after colonization by biofilm)

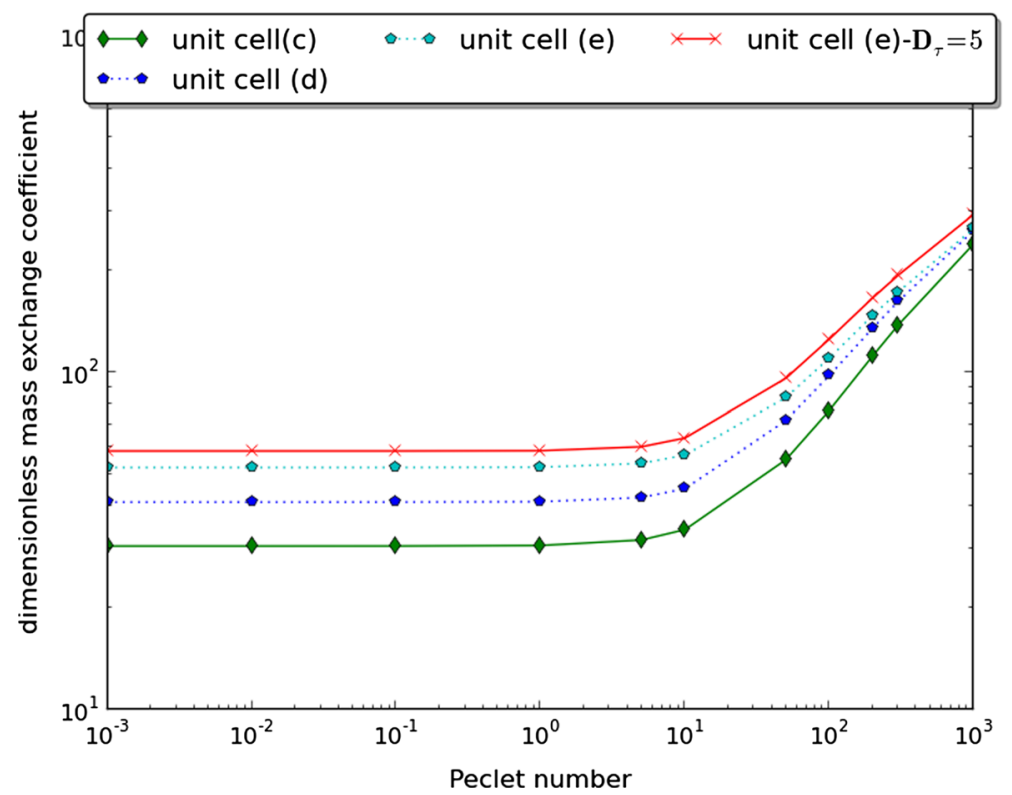

Fig. 8 Mass exchange coefficient: comparison of NAPL-wetting cases (before and after colonization by biofilm)

without biofilm — unit cells (d) and (e): after bacteria colonization). The influence of the solubilizing agents (i.e., the value of the partitioning coefficient $K_{\omega \gamma}$ at the NAPL-biofilm interface) will be investigated through two values of the diffusion ratio $\mathbf{D}_{\Gamma}$ fixed at 2.5 
$\left(K_{\omega \gamma}=5\right)$ and $5\left(K_{\omega \gamma}=10\right)$. Indeed, increasing $\mathbf{D}_{\Gamma}$ means increasing $K_{\omega \gamma}$, with all other parameters constant.

As we can see in Fig. 7, the influence of the biofilm development on dispersion phenomenon becomes observable at high Péclet numbers. If we observe the transverse dispersions, we note a low impact of the biofilm development contrary to the case of the longitudinal component. The more biofilm volume fraction increases (unit cell (d) $-\epsilon_{\omega}=0.011$ and unit cell (e) $-\epsilon_{\omega}=0.028$ ), the more longitudinal dispersion coefficient increases compared to the case without biofilm (unit cell(c) $-\epsilon_{\omega}=0$ ). This biomass development leads to an increase in lacunarity, as we can see in Table 1. For example, if lacunarity is increased of $25 \%$, the dispersion coefficient increases of $10 \%$. These results are related to the role of the biofilm activity that increases the NAPL solubility and hence the concentration gradients in the vicinity of NAPL blobs (Osswald et al. 1996). Note that the direct increase in $K_{\omega \gamma}$ reflects the same result.

For the second parameter under consideration, i.e., the mass exchange coefficient, it is clear that the presence of biofilm has a major impact especially at low Péclet numbers (Fig. 8). Increasing the biofilm volume fraction (or the value of $K_{\omega \gamma}$ ) favors an increase in the mass exchange coefficient. As mentioned above, the biofilm growth increases the concentration gradients and consequently the outward interfacial fluxes from the NAPL phase. Yet, this impact gets smaller with higher Péclet numbers. As the flow rate increases, external mass transfer limitations in the fluid phase tend to disappear which reduces the benefit of the highest solubility values in the biofilm phase where the mass transfer remains limited by diffusion (no advection within the biofilm).

\subsection{Impact of the Biofilm Development: Comparison of Non-wetting Cases (Before and After Colonization by Biofilm)_Configurations (a) and (b)}

Finally, the impact of bacterial growth on the effective properties of porous media is studied for the NAPL-non-wetting phase. The diffusion ratio $\mathbf{D}_{\Gamma}$ is kept constant at 2.5. Simulation results are reported in Figs. 9 and 10. As expected, we recover a behavior for both coefficients similar to the one observed for the NAPL-wetting case. Regarding the mass transfer coefficient, Fig. 10, it should be noted that the interfacial flux is practically the same at high Péclet numbers with or without bacterial activity. This result, already observed in the previous section, is more striking here. These results are in agreement with the trend observed in Fig. 5 where the mass exchange coefficient value was smaller in the wetting case (unit cell(c)) than for the non-wetting case (unit cell(a)). Advection prevails sooner for the non-wetting case and smoothes totally the impact of the biofilm colonization.

\subsection{Numerical Validation of the Macroscopic Model}

In this section, we validate the upscaling theory derived in this paper by a comparison between the macroscale model and direct numerical simulations (DNS) based on the pore scale model. We adopt the same approach that has been used in Golfier et al. (2009), Soulaine et al. (2011), Orgogozo et al. (2013). COMSOL Multiphysics Sofware ${ }^{\circledR}$ is used for all simulations. The geometry considered here is shown in Fig. 11. The unit cell (d) of dimension $L \times$ $L=12 \times 12 \mathrm{~mm}$ was used to construct this $2 \mathrm{D}$ geometry and was duplicated five times to obtain the final domain. The phases $(\beta, \omega$, and $\gamma)$ were generated in the same manner as the previous unit cells and volume fraction values are similar to the ones given in Table 1 for the unit cell (d). At the local scale, the physical problem will be two-dimensional and transient, whereas at the macroscale we will have a transient and one-dimensional problem. 


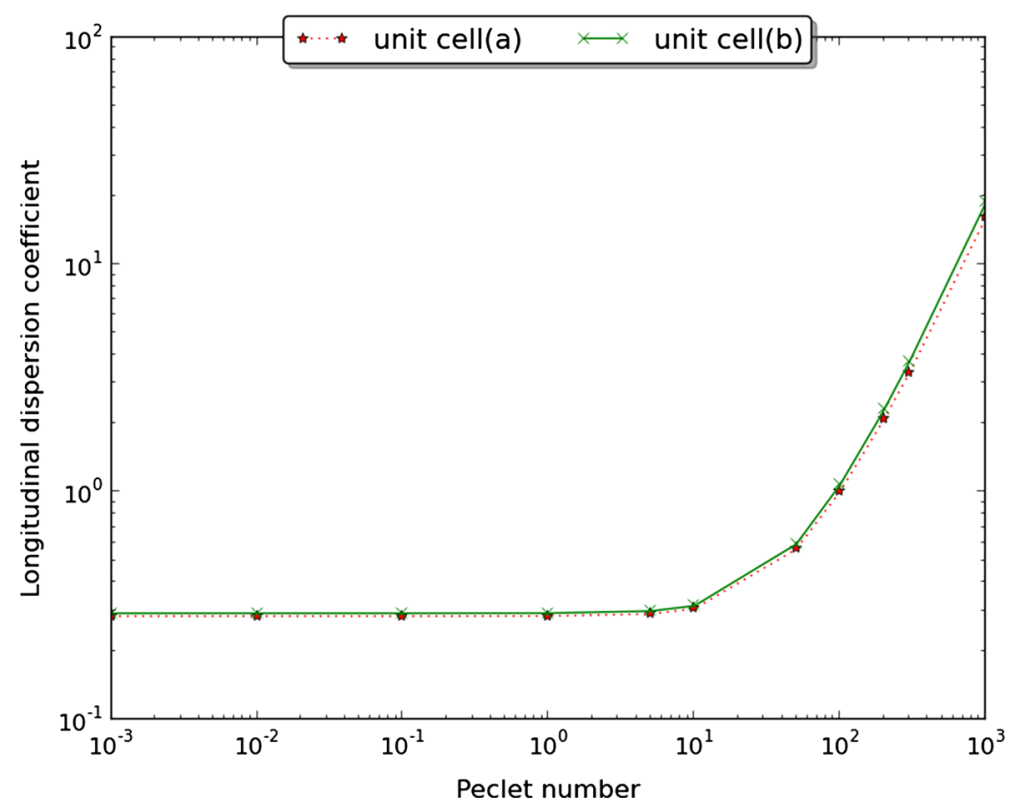

Fig. 9 Longitudinal dispersion coefficient: comparison of NAPL non-wetting cases (before and after colonization by biofilm)

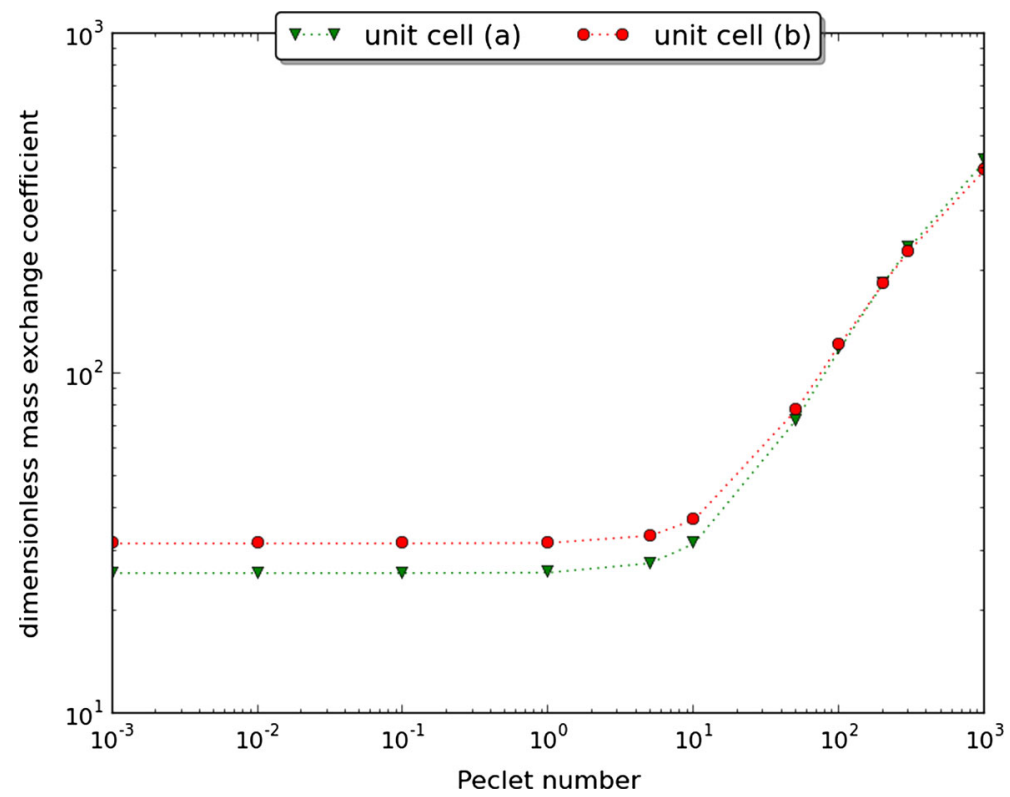

Fig. 10 Dimensionless mass exchange coefficient: comparison of NAPL non-wetting cases (before and after colonization by biofilm) 


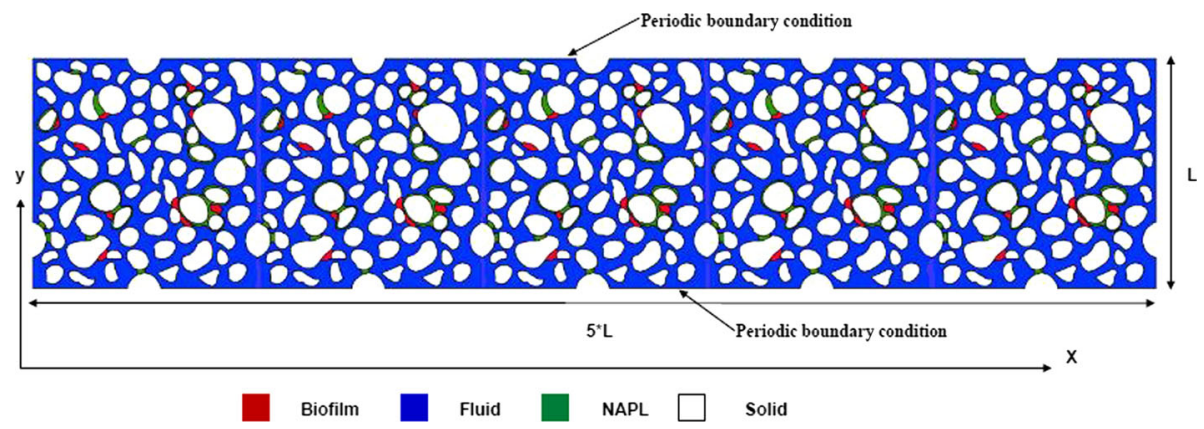

Fig. 11 Complex 2D geometry considered for numerical validation

Comparison will be focused on the steady-state spatial profiles of hydrocarbon concentration in the whole computational domain. Dynamics of NAPL blobs dissolution with time will not be simulated here. Since this dynamics is driven by the interfacial fluxes values, an investigation of concentration profiles is sufficient for comparison purposes.

The species $B$ is assumed to be in excess so that $c_{B \omega}^{*}=1$. In the following, we will keep the same reduced parameters as defined in Eq. 109. For the sake of simplicity, we will adopt a change in variable accordingly with

$$
c_{A K_{\omega}}^{\prime}=K_{\beta \omega} c_{A \omega}^{\prime}
$$

Thus, the dimensionless form of equations and associated boundary conditions under consideration at the pore scale reduces to:

$$
\frac{\partial c_{A \beta}^{\prime}}{\partial t^{\prime}}+P e_{A} \mathbf{v}_{\beta}^{\prime} \cdot \nabla c_{A \beta}^{\prime}=\nabla \cdot\left(\nabla c_{A \beta}^{\prime}\right) \quad \text { in the } \beta \text {-phase }
$$

B.C. $1 \mathbf{n}_{\beta \sigma} \cdot \nabla c_{A \beta}^{\prime}=0$ at $A_{\beta \sigma}$

B.C. $2 \mathbf{n}_{\omega \sigma} \cdot \mathbf{D}_{\Gamma} \cdot \nabla c_{A K_{\omega}}^{\prime}=0$ at $A_{\omega \sigma}$

B.C.3 $c_{A \beta}^{\prime}=c_{A K_{\omega}}^{\prime}$ at $A_{\beta \omega}$

B.C.4 $\mathbf{n}_{\beta \omega} \cdot \nabla c_{A \beta}^{\prime}=\mathbf{n}_{\beta \omega} \cdot \mathbf{D}_{\Gamma} \cdot \nabla c_{A K_{\omega}}^{\prime}$ at $A_{\beta \omega}$

B.C.5 $c_{A \beta}^{\prime}=1$ at $A_{\beta \gamma}$

B.C.6 $c_{A K_{\omega}}^{\prime}=1$ at $A_{\omega \gamma}$

$K_{\beta \omega}^{-1} \frac{\partial c_{A K_{\omega}}^{\prime}}{\partial t^{\prime}}=\nabla \cdot\left(\mathbf{D}_{\Gamma} \cdot \nabla c_{A K_{\omega}}^{\prime}\right)-D a_{A} \frac{c_{A K_{\omega}}^{\prime}}{K_{\beta \omega}+\frac{c_{A K_{\omega}}^{\prime}}{K_{A \omega}^{\prime}}+\frac{K_{\beta \omega}^{-1} c_{A K_{\omega}}^{\prime 2}}{K_{A \omega}^{\prime} K_{i}^{\prime}}}$ in the $\omega$-phase

where

$$
\begin{array}{r}
c_{A \omega}^{\prime}=\frac{c_{A \omega}}{c_{o}} ; \quad c_{A \beta}^{\prime}=\frac{c_{A \beta}}{c_{o}} ; \quad K_{A \omega}^{\prime}=\frac{K_{A \omega}}{c_{o}} ; \\
t^{\prime}=\frac{t D_{A \beta}}{L^{2}} ; \quad D a_{A}=\frac{\mu_{A \omega} \rho_{\omega} L^{2}}{D_{A \beta} K_{A \omega}} ; \quad K_{i}^{\prime}=\frac{K_{i}}{c_{o}} ;
\end{array}
$$


The additional boundary conditions are as follows:

- At the inlet: $c_{A \beta}=0$,

- At the outlet: $\mathbf{n}_{\mathbf{x}} \cdot\left(\mathbf{D}_{A \beta} \nabla c_{A \beta}+\mathbf{v}_{\beta} c_{A \beta}\right)=0$,

- Initial conditions: $c_{A \beta}^{\prime}=c_{A K_{\omega}}^{\prime}=0$.

In this analysis, we proceed by the following steps:

1. First, we solve the entire $2 \mathrm{D}$ microscopic problem on a total length of $5 \times L$. The velocity field is obtained by directly solving the Stokes equations (Eqs. 4, 13-15). We have specified a condition of pressure and concentration at the inlet. At the outlet, we applied a pressure condition and a convective flux (or Robin)-type boundary condition. Periodic conditions were applied for concentration and pressure on the other faces. The mesh refinement has been used to perform the mesh independence study. The final mesh is composed of 353,413 elements connecting 188,555 nodal points.

2. The second step consists in solving the closure problems on the unit cell (d) used to generate the domain for different Péclet numbers and calculate the effective properties. Then, once all the effective properties are known, we solve the 1D upscaled model, Eq. 110 , on a total length of $5 \times L$. The number of mesh nodes in the upscaled model is of 30721 .

3. Finally, the fields obtained from the pore scale simulations are averaged over cross sections to provide the $1 \mathrm{D}$ evolution of macroscale mass fractions $\left\langle c_{A \beta}\right\rangle^{\prime} \beta$ and compared to Darcy-scale simulations.

In the following, we present the typical results for three numerical conditions, respectively, $P e_{A}=0.01$ and $D a_{A}=0.01, P e_{A}=0.5$ and $D a_{A}=0.5$, and $P e_{A}=10$ and $D a_{A}=5$. $\mathbf{D}_{\Gamma}$ has been arbitrarily fixed at $0.8, K_{A \omega}^{\prime}$ at 0.5 and $K_{i}^{\prime}$ at 0.9 . The mass fraction value $\left\langle c_{A \beta}\right\rangle^{\prime} \beta$ along the $\mathrm{x}$-axis, which is used as comparison criterion for the simulations, is plotted in Fig. 12. For local equilibrium conditions $\left(P e_{A}=0.01\right.$ and $D a_{A}=0.01, P e_{A}=0.5$ and $D a_{A}=0.5$ ), we note a very good agreement (less than $3 \%$ of relative error) between the direct numerical simulation and the macroscale model. For such conditions, concentration gradients are sufficiently small at the fluid-biofilm interface so that the local mass equilibrium assumption can be ensured. On the contrary, when the Péclet or Damköhler number is too high, concentration gradients appear generated by advection or kinetics. Thus, non-equilibrium conditions prevail and our upscaled local equilibrium model is expected to fail. This is the case for $P e_{A}=10$ and $D a_{A}=5$ where the relative error increased up to $10 \%$. These observations are consistent with those obtained by Golfier et al. (2009) for a fluid-biofilm system.

A last point deserves to be investigated. In real-world applications, the correct knowledge of the aquifer features at the pore scale is usually missing. This is particularly true for nonsolid phases (biofilm or NAPL) where volume fractions (or saturations) are sometimes the only information available. In order to investigate the impact of the pore scale structure on the upscaled solution, we assumed that the distribution of the biofilm and NAPL phases is unknown. Consequently, we compared the same pore scale simulations with the Darcy-scale simulations performed for two different unit cells (unit cell (b) and (d)). The unit cell (d) keeps the correct volume fractions of each phase, but their distributions vary. For the two numerical conditions shown in Fig. $13\left(P e_{A}=0.01\right.$ and $D a_{A}=0.01, P e_{A}=10$ and $\left.D a_{A}=5\right)$, we observe a small impact of the unit cell geometry on the result, especially at low Péclet and Damköhler numbers since diffusive transfer is essentially driven by the volume fractions of each phase. When $P e_{A}$ and $D a_{A}$ increase, dependency of the upscaled solution to the pore scale features grows but remains relatively low. However, this small impact cannot be 

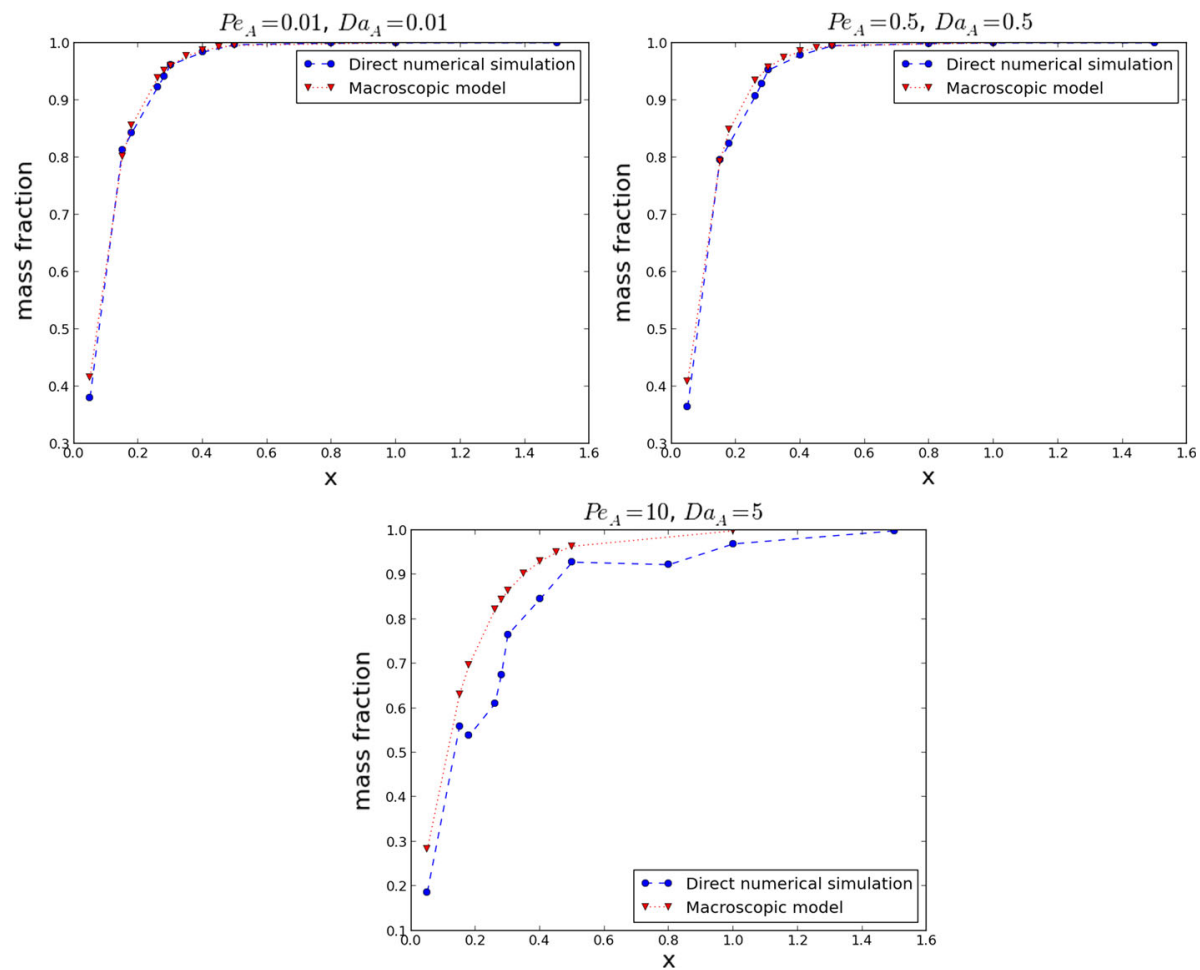

Fig. 12 Comparison of spatial profiles of concentration between DNS and the macroscopic model for a complex 2D geometry
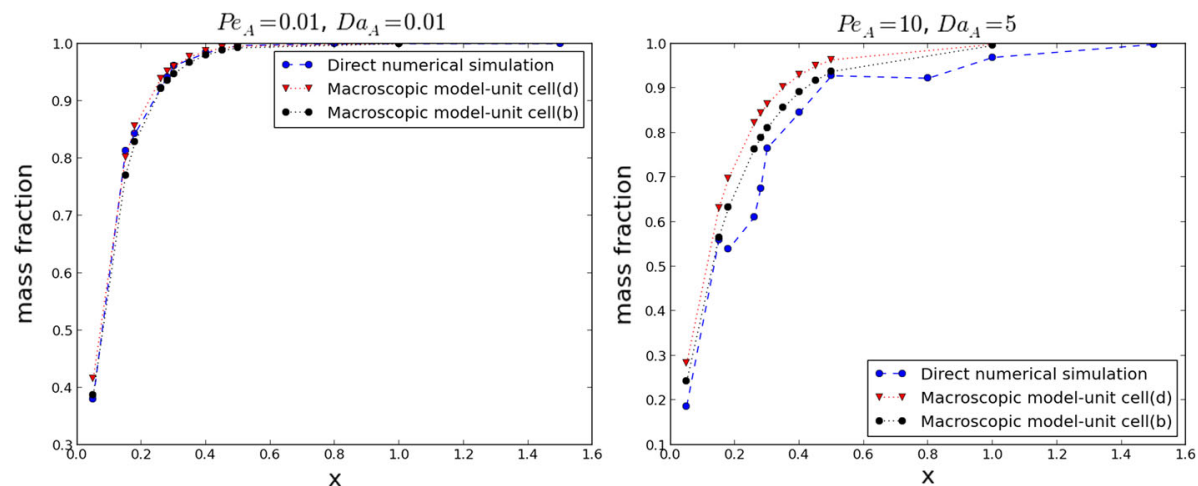

Fig. 13 Impact of the pore scale geometry on the upscaled concentration field

generalized to any geometry since there is essentially no a priori method to determine how much geometric structure is sufficient to adequately represent the transport processes within a unit cell. 


\section{Conclusion}

In this work, we developed an upscaled model of bio-enhanced NAPL dissolution in porous media using the volume-averaging method. We derived a set of two macroscale equations based on the local mass equilibrium assumption at the fluid-biofilm interface and the constraints associated with the hypothesis were previously defined.

The main assumptions on which this model is based are summarized below:

- We consider a saturated porous medium colonized by biofilm in the presence of a trapped hydrocarbon phase. Biodegradation reaction is described by Haldane kinetics (a large excess of electron acceptor is assumed).

- The hydrocarbon phase ( $\gamma$-phase) is assumed trapped residually into the porous matrix, and it is immobile.

- Biofilm growth and solute transport phenomena are uncoupled due to the separation of timescales for both processes.

- The macroscale mass balance equation is derived under local mass equilibrium condition at the fluid-biofilm interface.

Different types of 2D complex unit cells have been used to compute the mass exchange coefficient and the dispersion tensor components. The dissolution enhancement effect on the NAPL phase due to bacterial activity has been investigated. The results presented in this paper confirm the impact of microbial processes on the mass exchange coefficient and the dispersion tensor compared to the abiotic conditions. It has been shown that the pore scale architecture, function of the rock wettability, may significantly affect the biodisponibility of the NAPL phase and consequently its biodegradation (through the mass transfer coefficient value).

The representativity of the macroscale model was assessed by comparison with direct numerical simulation over a realistic $2 \mathrm{D}$ pore geometry. For low values of $P e_{A}$ and $D a_{A}$ numbers, i.e., for local equilibrium conditions, very good agreement between these solutions was obtained. For high values of $P e_{A}$ and $D a_{A}$ numbers, however, concentration gradients develop at the $\omega-\gamma$ interface and the relative error goes up to $10 \%$. This result suggests that the model is applicable with sufficient accuracy only for $P e_{A}<1$ and $D a_{A}<1$. Extension of this model to situations of non-equilibrium would require the development of a set of three coupled averaged equations, with one equation per phase.

In the present paper, we considered that the pore scale geometry was given a priori and closure problems and averaged equations were uncoupled due to a quasi-steady-state approximation. However, this pore scale geometry is a result of the dissolution process driven by the macroscale equations and changes with time due to biofilm growth and NAPL blobs dissolution. Integrating this coupling into an upscaling theory remains a challenging issue. A classical approach used in geochemistry to handle this complexity consists in assuming a direct relationship between the effective coefficients and porosity. However, pore scale surface patterns may evolve differently as a function of hydrodynamics. Pore scale simulations coupling flow and solute transport in evolving geometries (Benioug et al. 2015), biofilm growth model and NAPL dissolution are currently in progress and should provide interesting findings on this question.

To conclude, we would like to discuss about the coupling of our transport model to the microbial dynamics. This point has not been considered in this study; however, a number of approaches can be identified. Globally, two scenarios can be distinguished:

- Biofilm supported by aquifer grains is not thick enough to disturb groundwater flow, and hence, the biomass volume fraction does not vary across time. This is typical of 
usual subsurface conditions. Coupling with bacterial population dynamics (e.g., Monod equation) can be easily performed through a sequential procedure, and an example of this form of coupling with an upscaled transport equation can be found in Orgogozo et al. (2013). Application to the present bio-enhanced NAPL dissolution model is here straightforward.

- Biofilm growth cannot be neglected (e.g., bioplugging in the vicinity of the injection well during remediation strategy). This issue is by far more complex since an additional upscaled equation is required for predicting the changes in the volume fractions of each phase with time. Moreover, as mentioned above, changes in pore scale geometry would be lost through this approach, and the unit cell required for the calculation of the effective properties could not be updated in a direct way. This difficulty is well known for systems with process-dependent, evolving geometries, and further research is required on this point.

Acknowledgments This work was partially supported by the French National Research Agency (ANR) through the MOBIOPOR project, with the reference ANR-10-BLAN-0908 and by the French Scientific Interest Group-Industrial Wasteland (GISFI) program.

\section{Appendix 1: Derivation of the Concentration Deviations}

In this appendix, we determine the conservation equations for the deviation quantity by subtracting Eq. (51) from Eq. (64) and Eq. (52) from Eq. (65). From this, we derive the deviation problems by considering Gray's decomposition (Eqs. (27), (28)).

$\beta$-phase:

$$
\begin{aligned}
& \frac{\partial \tilde{c}_{A \beta}}{\partial t}+\mathbf{v}_{\beta} \cdot \nabla \tilde{c}_{A \beta}+\tilde{\mathbf{v}}_{\beta} \cdot \nabla\left\langle c_{A \beta}\right\rangle^{\beta}-\epsilon_{\beta}^{-1} \nabla \cdot\left\langle\tilde{\mathbf{v}}_{\beta} \tilde{c}_{A \beta}\right\rangle=\nabla \cdot\left(D_{A \beta} \nabla \tilde{c}_{A \beta}\right) \\
& -\epsilon_{\beta}^{-1} \nabla \cdot\left[D_{A \beta}\left(\frac{1}{V} \int_{A_{\beta \omega}} \mathbf{n}_{\beta \omega} \tilde{c}_{A \beta} \mathrm{d} A+\frac{1}{V} \int_{A_{\beta \gamma}} \mathbf{n}_{\beta \gamma} \tilde{c}_{A \beta} \mathrm{d} A+\frac{1}{V} \int_{A_{\beta \sigma}} \mathbf{n}_{\beta \sigma} \tilde{c}_{A \beta} \mathrm{d} A\right)\right] \\
& +\frac{\epsilon_{\beta}^{-1}}{V} \int_{A_{\beta \gamma}} \mathbf{n}_{\beta \gamma} \cdot \tilde{c}_{A \beta}\left(\mathbf{v}_{\beta}-\mathbf{w}_{\beta \gamma}\right) \mathrm{d} A+\frac{\epsilon_{\beta}^{-1}}{V} \int_{A_{\beta \omega}} \mathbf{n}_{\beta \omega} \cdot \tilde{c}_{A \beta}\left(\mathbf{v}_{\beta}-\mathbf{w}_{\beta \omega}\right) \mathrm{d} A \\
& +\frac{\epsilon_{\beta}^{-1}}{V} \int_{A_{\beta \sigma}} \mathbf{n}_{\beta \sigma} \cdot \tilde{c}_{A \beta}\left(\mathbf{v}_{\beta}-\mathbf{w}_{\beta \sigma}\right) \mathrm{d} A-\frac{\epsilon_{\beta}^{-1}}{V} \int_{A_{\beta \gamma}} \mathbf{n}_{\beta \gamma} \cdot D_{A \beta} \nabla \tilde{c}_{A \beta} \mathrm{d} A \\
& \quad-\frac{\epsilon_{\beta}^{-1}}{V} \int_{A_{\beta \omega}} \mathbf{n}_{\beta \omega} \cdot D_{A \beta} \nabla \tilde{c}_{A \beta} \mathrm{d} A-\frac{\epsilon_{\beta}^{-1}}{V} \int_{A_{\beta \sigma}} \mathbf{n}_{\beta \sigma} \cdot D_{A \beta} \nabla \tilde{c}_{A \beta} \mathrm{d} A
\end{aligned}
$$

$\omega$-phase:

$$
\begin{aligned}
& \frac{\partial \tilde{c}_{A \omega}}{\partial t}=\nabla \cdot\left(\mathbf{D}_{\mathbf{A} \omega} \cdot \nabla \tilde{c}_{A \omega}\right) \\
& -\epsilon_{\omega}^{-1} \nabla \cdot\left[\mathbf{D}_{\mathbf{A} \omega} \cdot\left(\frac{1}{V} \int_{A_{\omega \beta}} \mathbf{n}_{\omega \beta} \tilde{c}_{A \omega} \mathrm{d} A+\frac{1}{V} \int_{A_{\omega \gamma}} \mathbf{n}_{\omega \gamma} \tilde{c}_{A \omega} \mathrm{d} A+\frac{1}{V} \int_{A_{\omega \sigma}} \mathbf{n}_{\omega \sigma} \tilde{c}_{A \omega} \mathrm{d} A\right)\right] \\
& +\frac{\epsilon_{\omega}^{-1}}{V} \int_{A_{\omega \gamma}} \mathbf{n}_{\omega \gamma} \cdot \tilde{c}_{A \omega}\left(\mathbf{v}_{\omega}-\mathbf{w}_{\omega \gamma}\right) \mathrm{d} A+\frac{\epsilon_{\omega}^{-1}}{V} \int_{A_{\omega \beta}} \mathbf{n}_{\omega \beta} \cdot \tilde{c}_{A \omega}\left(\mathbf{v}_{\omega}-\mathbf{w}_{\omega \beta}\right) \mathrm{d} A
\end{aligned}
$$




$$
\begin{aligned}
& +\frac{\epsilon_{\omega}^{-1}}{V} \int_{A_{\omega \sigma}} \mathbf{n}_{\omega \sigma} \cdot \tilde{c}_{A \omega}\left(\mathbf{v}_{\omega}-\mathbf{w}_{\omega \sigma}\right) \mathrm{d} A-\frac{\epsilon_{\omega}^{-1}}{V} \int_{A_{\omega \gamma}} \mathbf{n}_{\omega \gamma} \cdot \mathbf{D}_{\mathbf{A} \omega} \cdot \nabla \tilde{c}_{A \omega} \mathrm{d} A \\
& -\frac{\epsilon_{\omega}^{-1}}{V} \int_{A_{\omega \beta}} \mathbf{n}_{\omega \beta} \cdot \mathbf{D}_{\mathbf{A} \omega} \cdot \nabla \tilde{c}_{A \omega} \mathrm{d} A-\frac{\epsilon_{\omega}^{-1}}{V} \int_{A_{\omega \sigma}} \mathbf{n}_{\omega \sigma} \mathbf{D}_{\mathbf{A} \omega} \nabla \tilde{c}_{A \omega} \mathrm{d} A+\left(R_{A \omega}-\left\langle R_{A \omega}\right\rangle^{\omega}\right)
\end{aligned}
$$

The spatial deviation concentration is evaluated at points other than the centroid of the averaging volume, adopting the classical following assumptions

$$
\begin{gathered}
\epsilon_{\beta}^{-1} \nabla \cdot\left(\frac{D_{A \beta}}{V} \int_{A_{\beta}} \mathbf{n}_{\beta \omega} \tilde{c}_{A \beta} \mathrm{d} A\right)<<\nabla .\left(D_{A \beta} \nabla \tilde{c}_{A \beta}\right) \\
\epsilon_{\omega}^{-1} \nabla \cdot\left(\frac{\mathbf{D}_{\mathbf{A} \omega}}{V} \int_{A_{\omega}} \mathbf{n}_{\omega \beta} \tilde{c}_{A \omega} \mathrm{d} A\right)<<\nabla .\left(\mathbf{D}_{\mathbf{A} \omega} \cdot \nabla \tilde{c}_{A \omega}\right) \\
\epsilon_{\beta}^{-1} \nabla \cdot\left\langle\tilde{\mathbf{v}}_{\beta} \tilde{c}_{A \beta}\right\rangle<<\tilde{\mathbf{v}}_{\beta} \cdot \nabla\left\langle c_{A \beta}\right\rangle^{\beta}
\end{gathered}
$$

Note that the reaction deviation term is generally negligible relative to the diffusive term (e.g., Golfier et al. 2009), i.e.,

$$
\left(R_{A \omega}-\left\langle R_{A \omega}\right\rangle^{\omega}\right)<<\nabla \cdot\left(D_{A \beta} \nabla \tilde{c}_{A \beta}\right)
$$

Under these assumptions, the equations for the $\beta$ - and $\omega$-phase become $\beta$-phase:

$$
\begin{aligned}
& \frac{\partial \tilde{c}_{A \beta}}{\partial t}+\mathbf{v}_{\beta} \cdot \nabla \tilde{c}_{A \beta}+\mathbf{Q}_{\beta} \cdot \nabla\left\langle c_{A \beta}\right\rangle^{\beta}=\nabla .\left(D_{A \beta} \nabla \tilde{c}_{A \beta}\right)+\frac{\epsilon_{\beta}^{-1}}{V} \int_{A_{\beta \gamma}} \mathbf{n}_{\beta \gamma} \cdot \tilde{c}_{A \beta}\left(\mathbf{v}_{\beta}-\mathbf{w}_{\beta \gamma}\right) \mathrm{d} A \\
& +\frac{\epsilon_{\beta}^{-1}}{V} \int_{A_{\beta \omega}} \mathbf{n}_{\beta \omega} \cdot \tilde{c}_{A \beta}\left(\mathbf{v}_{\beta}-\mathbf{w}_{\beta \omega}\right) \mathrm{d} A+\frac{\epsilon_{\beta}^{-1}}{V} \int_{A_{\beta \sigma}} \mathbf{n}_{\beta \sigma} \cdot \tilde{c}_{A \beta}\left(\mathbf{v}_{\beta}-\mathbf{w}_{\beta \sigma}\right) \mathrm{d} A \\
& -\frac{\epsilon_{\beta}^{-1}}{V} \int_{A_{\beta \gamma}} \mathbf{n}_{\beta \gamma} \cdot D_{A \beta} \nabla \tilde{c}_{A \beta} \mathrm{d} A-\frac{\epsilon_{\beta}^{-1}}{V} \int_{A_{\beta \omega}} \mathbf{n}_{\beta \omega} \cdot D_{A \beta} \nabla \tilde{c}_{A \beta} \mathrm{d} A \\
& -\frac{\epsilon_{\beta}^{-1}}{V} \int_{A_{\beta \sigma}} \mathbf{n}_{\beta \sigma} \cdot D_{A \beta} \nabla \tilde{c}_{A \beta} \mathrm{d} A
\end{aligned}
$$

$\omega$-phase:

$$
\begin{aligned}
& \frac{\partial \tilde{c}_{A \omega}}{\partial t}=\nabla \cdot\left(\mathbf{D}_{\mathbf{A} \omega} \cdot \nabla \tilde{c}_{A \omega}\right)+\frac{\epsilon_{\omega}^{-1}}{V} \int_{A_{\omega \gamma}} \mathbf{n}_{\omega \gamma} \cdot \tilde{c}_{A \omega}\left(\mathbf{v}_{\omega}-\mathbf{w}_{\omega \gamma}\right) \mathrm{d} A \\
& +\frac{\epsilon_{\omega}^{-1}}{V} \int_{A_{\omega \beta}} \mathbf{n}_{\omega \beta} \cdot \tilde{c}_{A \omega}\left(\mathbf{v}_{\omega}-\mathbf{w}_{\omega \beta}\right) \mathrm{d} A+\frac{\epsilon_{\omega}^{-1}}{V} \int_{A_{\omega \sigma}} \mathbf{n}_{\omega \sigma} \cdot \tilde{c}_{A \omega}\left(\mathbf{v}_{\omega}-\mathbf{w}_{\omega \sigma}\right) \mathrm{d} A \\
& -\frac{\epsilon_{\omega}^{-1}}{V} \int_{A_{\omega \gamma}} \mathbf{n}_{\omega \gamma} \cdot \mathbf{D}_{\mathbf{A} \omega} \cdot \nabla \tilde{c}_{A \omega} \mathrm{d} A-\frac{\epsilon_{\omega}^{-1}}{V} \int_{A_{\omega \beta}} \mathbf{n}_{\omega \beta} \cdot \mathbf{D}_{\mathbf{A} \omega} \cdot \nabla \tilde{c}_{A \omega} \mathrm{d} A \\
& -\frac{\epsilon_{\omega}^{-1}}{V} \int_{A_{\omega \sigma}} \mathbf{n}_{\omega \sigma} \cdot \mathbf{D}_{\mathbf{A} \omega} \cdot \nabla \tilde{c}_{A \omega} \mathrm{d} A
\end{aligned}
$$

The boundary conditions given by Eqs. (5)-(10) are necessary to complete our statement of the deviation problem. We can use the spatial decomposition given by Eqs. (27) and (28) for developing the boundary conditions associated with this deviation problem 


$$
\begin{aligned}
& \text { B.C. } 1-\mathbf{n}_{\beta \sigma} \cdot D_{A \beta} \nabla\left\langle c_{A \beta}\right\rangle^{\beta}=\mathbf{n}_{\beta \sigma} \cdot D_{A \beta} \nabla \tilde{c}_{A \beta} \quad \text { at } A_{\beta \sigma} \\
& \text { B.C. }-\mathbf{n}_{\omega \sigma} \cdot \mathbf{D}_{\mathbf{A} \omega} \cdot \nabla\left\langle c_{A \omega}\right\rangle^{\beta}=\mathbf{n}_{\omega \sigma} \cdot \mathbf{D}_{\mathbf{A} \omega} \cdot \nabla \tilde{c}_{A \omega} \text { at } A_{\omega \sigma} \\
& \text { B.C.3 } \tilde{c}_{A \beta}+\left\langle c_{A \beta}\right\rangle^{\beta}=K_{\beta \omega} \tilde{c}_{A \omega}+K_{\beta \omega}\left\langle c_{A \omega}\right\rangle^{\omega} \text { at } A_{\beta \omega} \\
& \text { B.C.4 } \mathbf{n}_{\beta \omega} \cdot\left(\left\langle c_{A \beta}\right\rangle^{\beta}\left(\mathbf{v}_{\beta}-\mathbf{w}_{\beta \omega}\right)-D_{A \beta} \nabla\left\langle c_{A \beta}\right\rangle^{\beta}\right)+\mathbf{n}_{\beta \omega} \cdot\left(\tilde{c}_{A \beta}\left(\mathbf{v}_{\beta}-\mathbf{w}_{\beta \omega}\right)-D_{A \beta} \nabla \tilde{c}_{A \beta}\right) \\
& =\mathbf{n}_{\beta \omega} \cdot\left(\left\langle c_{A \omega}\right\rangle^{\omega}\left(\mathbf{v}_{\omega}-\mathbf{w}_{\omega \beta}\right)-\mathbf{D}_{\mathbf{A} \omega} \cdot \nabla\left\langle c_{A \omega}\right\rangle^{\omega}\right) \\
& +\mathbf{n}_{\beta \omega} \cdot\left(\tilde{c}_{A \omega}\left(\mathbf{v}_{\omega}-\mathbf{w}_{\omega \beta}\right)-\mathbf{D}_{\mathbf{A} \omega} \cdot \nabla \tilde{c}_{A \omega}\right) \text { at } A_{\beta \omega} \\
& \text { B.C.5 } \tilde{c}_{A \omega}=c_{A \omega}^{\mathrm{eq}}-\left\langle c_{A \omega}\right\rangle^{\omega} \text { at } A_{\omega \gamma} \\
& \text { B.C.6 } \tilde{c}_{A \beta}=c_{A \beta}^{\mathrm{eq}}-\left\langle c_{A \beta}\right\rangle^{\beta} \text { at } A_{\beta \gamma} \\
& \text { B.C.7(Periodicity) } \quad \tilde{c}_{A \beta}\left(\mathbf{r}+l_{i}\right)=\tilde{c}_{A \beta}(\mathbf{r}), \quad i=1,2,3 \\
& \text { B.C.8(Periodicity) } \tilde{c}_{A \omega}\left(\mathbf{r}+l_{i}\right)=\tilde{c}_{A \omega}(\mathbf{r}), \quad i=1,2,3 \\
& \text { B.C. } 9\left\langle\tilde{c}_{A \beta}\right\rangle^{\beta}=0 \\
& \text { B.C.10 }\left\langle\tilde{c}_{A \omega}\right\rangle^{\omega}=0
\end{aligned}
$$

At this point, an initial condition should be theoretically required to get a complete problem. Anyway, this is not a concern since we are only interested in the quasi-steady-state solution of the deviation problem. We apply the following assumptions

$$
\frac{\partial \tilde{c}_{A \beta}}{\partial t}<<\nabla .\left(D_{A \beta} \nabla \tilde{c}_{A \beta}\right) \quad \frac{\partial \tilde{c}_{A \omega}}{\partial t}<<\nabla .\left(\mathbf{D}_{\mathbf{A} \omega} \cdot \nabla \tilde{c}_{A \omega}\right)
$$

The timescale constraint for the quasi-steady assumption is discussed in more detail in Quintard and Whitaker (1993).

Using a quasi-steady-state approximation and under conditions of local mass equilibrium (Eq. (59)), we finally obtain the set of equations governing the concentration deviations $\beta$-phase:

$$
\begin{aligned}
& \mathbf{v}_{\beta} \cdot \nabla \tilde{c}_{A \beta}+\tilde{\mathbf{v}_{\beta}} \cdot \nabla\left\langle c_{A \beta}\right\rangle^{\beta}=\nabla \cdot\left(D_{A \beta} \nabla \tilde{c}_{A \beta}\right)+\frac{\epsilon_{\beta}^{-1}}{V} \int_{A_{\beta \gamma}} \mathbf{n}_{\beta \gamma} \cdot \tilde{c}_{A \beta}\left(\mathbf{v}_{\beta}-\mathbf{w}_{\beta \gamma}\right) \mathrm{d} A \\
& +\frac{\epsilon_{\beta}^{-1}}{V} \int_{A_{\beta \omega}} \mathbf{n}_{\beta \omega} \cdot \tilde{c}_{A \beta}\left(\mathbf{v}_{\beta}-\mathbf{w}_{\beta \omega}\right) \mathrm{d} A+\frac{\epsilon_{\beta}^{-1}}{V} \int_{A_{\beta \sigma}} \mathbf{n}_{\beta \sigma} \cdot \tilde{c}_{A \beta}\left(\mathbf{v}_{\beta}-\mathbf{w}_{\beta \sigma}\right) \mathrm{d} A \\
& -\frac{\epsilon_{\beta}^{-1}}{V} \int_{A_{\beta \gamma}} \mathbf{n}_{\beta \gamma} \cdot D_{A \beta} \nabla \tilde{c}_{A \beta} \mathrm{d} A-\frac{\epsilon_{\beta}^{-1}}{V} \int_{A_{\beta \omega}} \mathbf{n}_{\beta \omega} \cdot D_{A \beta} \nabla \tilde{c}_{A \beta} \mathrm{d} A \\
& -\frac{\epsilon_{\beta}^{-1}}{V} \int_{A_{\beta \sigma}} \mathbf{n}_{\beta \sigma} \cdot D_{A \beta} \nabla \tilde{c}_{A \beta} \mathrm{d} A
\end{aligned}
$$

B.C.1 $-\underbrace{\mathbf{n}_{\beta \sigma} \cdot D_{A \beta} \cdot \nabla\left\langle c_{A \beta}\right\rangle^{\beta}}_{\text {Source }}=\mathbf{n}_{\beta \sigma} \cdot D_{A \beta} \nabla \tilde{c}_{A \beta} \quad$ at $A_{\beta \sigma}$

B.C.2 $-\underbrace{\mathbf{n}_{\omega \sigma} \cdot \mathbf{D}_{\mathbf{A} \omega} K_{\beta \omega}^{-1} \cdot \nabla\left\langle c_{A \beta}\right\rangle^{\beta}}_{\text {Source }}=\mathbf{n}_{\omega \sigma} \cdot \mathbf{D}_{\mathbf{A} \omega} \cdot \nabla \tilde{c}_{A \omega} \quad$ at $A_{\omega \sigma}$

B.C.3 $\tilde{c}_{A \beta}=K_{\beta \omega} \tilde{c}_{A \omega}$ at $A_{\beta \omega}$

B.C.4 $\underbrace{\mathbf{n}_{\beta \omega} \cdot\left(\left(\mathbf{v}_{\beta}-\mathbf{w}_{\beta \omega}\right)-K_{\beta \omega}^{-1}\left(\mathbf{v}_{\omega}-\mathbf{w}_{\omega \beta}\right)\right)\left\langle c_{A \beta}\right\rangle^{\beta}}+\mathbf{n}_{\beta \omega} \cdot\left(\tilde{c}_{A \beta}\left(\mathbf{v}_{\beta}-\mathbf{w}_{\beta \omega}\right)-D_{A \beta} \nabla \tilde{c}_{A \beta}\right)$ 


$$
=\underbrace{\mathbf{n}_{\beta \omega} \cdot\left(D_{A \beta} \mathbf{I}-K_{\beta \omega}^{-1} D_{A \omega}\right) \cdot \nabla\left\langle c_{A \beta}\right\rangle^{\beta}}_{\text {Source }}+\mathbf{n}_{\beta \omega} \cdot\left(\tilde{c}_{A \omega}\left(\mathbf{v}_{\omega}-\mathbf{w}_{\omega \beta}\right)-\mathbf{D}_{\mathbf{A} \omega} \cdot \nabla \tilde{c}_{A \omega}\right) \text { at } A_{\beta \omega}
$$

B.C.5 $\tilde{c}_{A \omega}=\underbrace{K_{\beta \omega}^{-1}\left(c_{A \beta}^{\mathrm{eq}}-\left\langle c_{A \beta}\right\rangle^{\beta}\right)}_{\text {Source }}$ at $A_{\omega \gamma}$

B.C.6 $\tilde{c}_{A \beta}=\underbrace{c_{A \beta}^{\mathrm{eq}}-\left\langle c_{A \beta}\right\rangle^{\beta}}_{\text {Source }}$ at $A_{\beta \gamma}$

B.C.7(Periodicity) $\tilde{c}_{A \beta}\left(\mathbf{r}+l_{i}\right)=\tilde{c}_{A \beta}(\mathbf{r}), \quad i=1,2,3$

B.C.8(Periodicity) $\quad \tilde{c}_{A \omega}\left(\mathbf{r}+l_{i}\right)=\tilde{c}_{A \omega}(\mathbf{r}), \quad i=1,2,3$

B.C.9 $\left\langle\tilde{c}_{A \beta}\right\rangle^{\beta}=0$

B.C.10 $\left\langle\tilde{c}_{A \omega}\right\rangle^{\omega}=0$

$\omega$-phase:

$$
\begin{aligned}
& \nabla .\left(\mathbf{D}_{\mathbf{A} \omega} . \nabla \tilde{c}_{A \omega}\right)=\frac{\epsilon_{\omega}^{-1}}{V} \int_{A_{\omega \gamma}} \mathbf{n}_{\omega \gamma} \cdot \tilde{c}_{A \omega}\left(\mathbf{v}_{\omega}-\mathbf{w}_{\omega \gamma}\right) \mathrm{d} A+\frac{\epsilon_{\omega}^{-1}}{V} \int_{A_{\omega \beta}} \mathbf{n}_{\omega \beta} \cdot \tilde{c}_{A \omega}\left(\mathbf{v}_{\omega}-\mathbf{w}_{\omega \beta}\right) \mathrm{d} A \\
& +\frac{\epsilon_{\omega}^{-1}}{V} \int_{A_{\omega \sigma}} \mathbf{n}_{\omega \sigma} \cdot \tilde{c}_{A \omega}\left(\mathbf{v}_{\omega}-\mathbf{w}_{\omega \sigma}\right) \mathrm{d} A-\frac{\epsilon_{\omega}^{-1}}{V} \int_{A_{\omega \gamma}} \mathbf{n}_{\omega \gamma} \cdot \mathbf{D}_{\mathbf{A} \omega} \cdot \nabla \tilde{c}_{A \omega} \mathrm{d} A \\
& -\frac{\epsilon_{\omega}^{-1}}{V} \int_{A_{\omega \beta}} \mathbf{n}_{\omega \beta} \cdot \mathbf{D}_{\mathbf{A} \omega} \cdot \nabla \tilde{c}_{A \omega} \mathrm{d} A-\frac{\epsilon_{\omega}^{-1}}{V} \int_{A_{\omega \sigma}} \mathbf{n}_{\omega \sigma} \cdot \mathbf{D}_{\mathbf{A} \omega} \cdot \nabla \tilde{c}_{A \omega} \mathrm{d} A
\end{aligned}
$$

\section{Appendix 2: Development of the Closure Problems}

The concentration deviations described by Eqs. (160) and (171) lead to the development of the closure problems. The simplified form of these equations (dimensionless form) is presented in Sect. 5.1.

The closure problem for the vector closure variable is given by

\section{Problem I}

$$
\begin{aligned}
& \beta \text {-phase : } \quad \mathbf{v}_{\beta} \cdot \nabla \mathbf{b}_{\beta}+\tilde{\mathbf{v}_{\beta}}=\nabla \cdot\left(D_{A \beta} \nabla \mathbf{b}_{\beta}\right)+\epsilon_{\beta}^{-1} \mathbf{U}_{\beta} \\
& \text { B.C.1 }-\mathbf{n}_{\beta \sigma} \cdot \nabla \mathbf{b}_{\beta}=\mathbf{n}_{\beta \sigma} \quad \text { at } A_{\beta \sigma} \\
& \text { B.C. } 2-\mathbf{n}_{\omega \sigma} \cdot \nabla \mathbf{b}_{\omega}=\mathbf{n}_{\omega \sigma} \quad \text { at } A_{\omega \sigma} \\
& \text { B.C. } 3 \mathbf{b}_{\beta}=\mathbf{b}_{\omega} \text { at } A_{\beta \omega} \\
& \text { B.C.4 } \mathbf{n}_{\beta \omega} \cdot\left(\mathbf{b}_{\beta}\left(\mathbf{v}_{\beta}-\mathbf{w}_{\beta \omega}\right)-D_{A \beta} \nabla \mathbf{b}_{\beta}\right)=\mathbf{n}_{\beta \omega} \cdot\left(D_{A \beta} \mathbf{I}-K_{\beta \omega}^{-1} D_{A \omega}\right) \\
& +\mathbf{n}_{\beta \omega} \cdot\left(K_{\beta \omega}^{-1} \mathbf{b}_{\omega}\left(\mathbf{v}_{\omega}-\mathbf{w}_{\omega \beta}\right)-\mathbf{D}_{\mathbf{A} \omega} K_{\beta \omega}^{-1} \nabla \mathbf{b}_{\omega}\right) \text { at } A_{\beta \omega} \\
& \text { B.C.5 } \mathbf{b}_{\beta}=0 \text { at } A_{\beta \gamma} \\
& \text { B.C.6 } \mathbf{b}_{\omega}=0 \text { at } A_{\omega \gamma} \\
& \text { B.C.7(Periodicity) } \mathbf{b}_{\beta}\left(\mathbf{r}+l_{i}\right)=\mathbf{b}_{\beta}(\mathbf{r}), i=1,2,3 \quad i=1,2,3 \\
& \text { B.C.8(Periodicity) } \mathbf{b}_{\omega}\left(\mathbf{r}+l_{i}\right)=\mathbf{b}_{\omega}(\mathbf{r}), i=1,2,3 \quad i=1,2,3 \\
& \text { B.C.9 }\left\langle\mathbf{b}_{\beta}\right\rangle^{\beta}=0 \text { at } A_{\beta e} \\
& \text { B.C.10 }\left\langle\mathbf{b}_{\omega}\right\rangle^{\omega}=0 \text { at } A_{\omega e}
\end{aligned}
$$




$$
\begin{aligned}
& \omega \text {-phase : } \nabla .\left(\mathbf{D}_{\mathbf{A} \omega} \cdot \nabla \mathbf{b}_{\omega}\right)=\epsilon_{\omega}^{-1} \mathbf{U}_{\omega} \\
& \mathbf{U}_{\beta}=\frac{1}{V} \int_{A_{\beta \gamma}} \mathbf{n}_{\beta \gamma} \cdot \mathbf{b}_{\beta}\left(\mathbf{v}_{\beta}-\mathbf{w}_{\beta \gamma}\right) \mathrm{d} A+\frac{1}{V} \int_{A_{\beta \omega}} \mathbf{n}_{\beta \omega} \cdot \mathbf{b}_{\beta}\left(\mathbf{v}_{\beta}-\mathbf{w}_{\beta \omega}\right) \mathrm{d} A \\
& +\frac{1}{V} \int_{A_{\beta \sigma}} \mathbf{n}_{\beta \sigma} \cdot \mathbf{b}_{\beta}\left(\mathbf{v}_{\beta}-\mathbf{w}_{\beta \sigma}\right) \mathrm{d} A-\frac{1}{V} \int_{A_{\beta \gamma}} \mathbf{n}_{\beta \gamma} \cdot D_{A \beta} \nabla \mathbf{b}_{\beta} \mathrm{d} A \\
& -\frac{1}{V} \int_{A_{\beta \omega}} \mathbf{n}_{\beta \omega} \cdot D_{A \beta} \nabla \mathbf{b}_{\beta} \mathrm{d} A-\frac{1}{V} \int_{A_{\beta \sigma}} \mathbf{n}_{\beta \sigma} \cdot D_{A \beta} \nabla \mathbf{b}_{\beta} \mathrm{d} A \\
& \mathbf{U}_{\omega}=\frac{1}{V} \int_{A_{\omega \gamma}} \mathbf{n}_{\omega \gamma} \cdot \mathbf{b}_{\omega}\left(\mathbf{v}_{\omega}-\mathbf{w}_{\omega \gamma}\right) \mathrm{d} A+\frac{1}{V} \int_{A_{\omega \beta}} \mathbf{n}_{\omega \beta} \cdot \mathbf{b}_{\omega}\left(\mathbf{v}_{\omega}-\mathbf{w}_{\omega \beta}\right) \mathrm{d} A \\
& +\frac{1}{V} \int_{A_{\omega \sigma}} \mathbf{n}_{\omega \sigma} \cdot \mathbf{b}_{\omega}\left(\mathbf{v}_{\omega}-\mathbf{w}_{\omega \sigma}\right) \mathrm{d} A-\frac{1}{V} \int_{A_{\omega \gamma}} \mathbf{n}_{\omega \gamma} \cdot \mathbf{D}_{\mathrm{A} \omega} \cdot \nabla \mathbf{b}_{\omega} \mathrm{d} A \\
& -\frac{1}{V} \int_{A_{\omega \beta}} \mathbf{n}_{\omega \beta} \cdot \mathbf{D}_{\mathrm{A} \omega} \cdot \nabla \mathbf{b}_{\omega} \mathrm{d} A-\frac{1}{V} \int_{A_{\omega \sigma}} \mathbf{n}_{\omega \sigma} \cdot \mathbf{D}_{\mathbf{A} \omega \cdot} \cdot \nabla \mathbf{b}_{\omega} \mathrm{d} A
\end{aligned}
$$

The closure problem for the scalar closure variable is given by

\section{Problem II}

$$
\begin{aligned}
& \beta \text {-phase : } \quad \mathbf{v}_{\beta} \cdot \nabla s_{\beta}=\nabla \cdot\left(D_{A \beta} \nabla s_{\beta}\right)+\epsilon_{\beta}^{-1} \mathbf{S}_{\beta} \\
& \text { B.C. } 1-\mathbf{n}_{\beta \sigma} \cdot \nabla s_{\beta}=0 \text { at } A_{\beta \sigma} \\
& \text { B.C. } 2-\mathbf{n}_{\omega \sigma} \cdot \nabla s_{\omega}=0 \text { at } A_{\omega \sigma} \\
& \text { B.C.3 } s_{\beta}=s_{\omega} \text { at } A_{\beta \omega} \\
& \text { B.C.4 } \mathbf{n}_{\beta \omega} \cdot\left(\left(\mathbf{v}_{\beta}-\mathbf{w}_{\beta \omega}\right)-K_{\beta \omega}^{-1}\left(\mathbf{v}_{\omega}-\mathbf{w}_{\omega \beta}\right)\right)\left\langle c_{A \beta}\right\rangle^{\beta}+ \\
& \mathbf{n}_{\beta \omega} \cdot\left(s_{\beta}\left(c_{A \beta}^{\mathrm{eq}}-\left\langle c_{A \beta}\right\rangle^{\beta}\right)\left(\mathbf{v}_{\beta}-\mathbf{w}_{\beta \omega}\right)-D_{A \beta} \nabla s_{\beta}\right)= \\
& \mathbf{n}_{\beta \omega} \cdot\left(s_{\omega} K_{\beta \omega}^{-1}\left(c_{A \beta}^{\mathrm{eq}}-\left\langle c_{A \beta}\right\rangle^{\beta}\right)\left(\mathbf{v}_{\omega}-\mathbf{w}_{\omega \beta}\right)-\mathbf{D}_{\mathrm{A} \omega} K_{\beta \omega}^{-1} \nabla s_{\omega}\right) \text { at } A_{\beta \omega} \\
& \text { B.C.5 } s_{\beta}=1 \text { at } A_{\beta \gamma} \\
& \text { B.C.6 } s_{\omega}=1 \text { at } A_{\omega \gamma} \\
& \text { B.C.7(Periodicity) } s_{\beta}\left(\mathbf{r}+l_{i}\right)=s_{\beta}(\mathbf{r}), i=1,2,3 \quad i=1,2,3 \\
& \text { B.C.8(Periodicity) } s_{\omega}\left(\mathbf{r}+l_{i}\right)=s_{\omega}(\mathbf{r}), i=1,2,3 \quad i=1,2,3 \\
& \text { B.C. } 9\left\langle s_{\beta}\right\rangle^{\beta}=0 \\
& \text { B.C.10 }\left\langle s_{\omega}\right\rangle^{\omega}=0 \\
& \omega \text {-phase : } \nabla .\left(\mathbf{D}_{\mathbf{A} \omega} \cdot \nabla s_{\omega}\right)=\epsilon_{\omega}^{-1} \mathbf{S}_{\omega} \\
& \mathbf{S}_{\beta}=\frac{1}{V} \int_{A_{\beta \gamma}} \mathbf{n}_{\beta \gamma} \cdot s_{\beta}\left(\mathbf{v}_{\beta}-\mathbf{w}_{\beta \gamma}\right) \mathrm{d} A+\frac{1}{V} \int_{A_{\beta \omega}} \mathbf{n}_{\beta \omega} \cdot s_{\beta}\left(\mathbf{v}_{\beta}-\mathbf{w}_{\beta \omega}\right) \mathrm{d} A \\
& +\frac{1}{V} \int_{A_{\beta \sigma}} \mathbf{n}_{\beta \sigma} \cdot s_{\beta}\left(\mathbf{v}_{\beta}-\mathbf{w}_{\beta \sigma}\right) \mathrm{d} A-\frac{1}{V} \int_{A_{\beta \gamma}} \mathbf{n}_{\beta \gamma} D_{A \beta} \cdot \nabla s_{\beta} \mathrm{d} A \\
& -\frac{1}{V} \int_{A_{\beta \omega}} \mathbf{n}_{\beta \omega} D_{A \beta} \cdot \nabla s_{\beta} \mathrm{d} A-\frac{1}{V} \int_{A_{\beta \sigma}} \mathbf{n}_{\beta \sigma} D_{A \beta} \cdot \nabla s_{\beta} \mathrm{d} A \\
& \mathbf{S}_{\omega}=\frac{1}{V} \int_{A_{\omega \gamma}} \mathbf{n}_{\omega \gamma} \cdot s_{\omega}\left(\mathbf{v}_{\omega}-\mathbf{w}_{\omega \gamma}\right) \mathrm{d} A+\frac{1}{V} \int_{A_{\omega \beta}} \mathbf{n}_{\omega \beta} \cdot s_{\omega}\left(\mathbf{v}_{\omega}-\mathbf{w}_{\omega \beta}\right) \mathrm{d} A
\end{aligned}
$$




$$
\begin{aligned}
& +\frac{1}{V} \int_{A_{\omega \sigma}} \mathbf{n}_{\omega \sigma} \cdot s_{\omega}\left(\mathbf{v}_{\omega}-\mathbf{w}_{\omega \sigma}\right) \mathrm{d} A-\frac{1}{V} \int_{A_{\omega \gamma}} \mathbf{n}_{\omega \gamma} \cdot \mathbf{D}_{\mathrm{A} \omega} \cdot \nabla s_{\omega} \mathrm{d} A \\
& -\frac{1}{V} \int_{A_{\omega \beta}} \mathbf{n}_{\omega \beta} \cdot \mathbf{D}_{\mathbf{A} \omega} \cdot \nabla s_{\omega} \mathrm{d} A-\frac{1}{V} \int_{A_{\omega \sigma}} \mathbf{n}_{\omega \sigma} \cdot \mathbf{D}_{\mathbf{A} \omega} \cdot \nabla s_{\omega} \mathrm{d} A
\end{aligned}
$$

To simplify the closure problems, the flux terms involving $\left(\mathbf{v}_{\beta}-\mathbf{w}_{\beta \omega}\right)$ are neglected in front of the diffusive fluxes. As an example, B.C.4 (Eq. (176)) in Problem I becomes:

$$
-\mathbf{n}_{\beta \omega} \cdot D_{A \beta} \nabla \mathbf{b}_{\beta}=\mathbf{n}_{\beta \omega} \cdot\left(D_{A \beta} \mathbf{I}-K_{\beta \omega}^{-1} D_{A \omega}\right)-\mathbf{n}_{\beta \omega} \cdot \mathbf{D}_{\mathbf{A} \omega} \cdot K_{\beta \omega}^{-1} \nabla \mathbf{b}_{\omega}
$$

Under the previous assumption, the closure problems can be rewritten as:

\section{Problem I}

$$
\begin{aligned}
& \beta \text {-phase : } \quad \mathbf{v}_{\beta} \cdot \nabla \mathbf{b}_{\beta}+\tilde{\mathbf{v}_{\beta}}=\nabla \cdot\left(D_{A \beta} \nabla \mathbf{b}_{\beta}\right)+\epsilon_{\beta}^{-1} \mathbf{U}_{\beta} \\
& \text { B.C.1 }-\mathbf{n}_{\beta \sigma} \cdot \nabla \mathbf{b}_{\beta}=\mathbf{n}_{\beta \sigma} \quad \text { at } A_{\beta \sigma} \\
& \text { B.C.2 }-\mathbf{n}_{\omega \sigma} \cdot \nabla \mathbf{b}_{\omega}=\mathbf{n}_{\omega \sigma} \quad \text { at } A_{\omega \sigma} \\
& \text { B.C.3 } \mathbf{b}_{\beta}=\mathbf{b}_{\omega} \quad \text { at } A_{\beta \omega} \\
& \text { B.C.4 }-\mathbf{n}_{\beta \omega} . D_{A \beta} \nabla \mathbf{b}_{\beta}=\mathbf{n}_{\beta \omega} \cdot\left(D_{A \beta} \mathbf{I}-K_{\beta \omega}^{-1} D_{A \omega}\right)-\mathbf{n}_{\beta \omega} \cdot \mathbf{D}_{\mathbf{A} \omega} \cdot K_{\beta \omega}^{-1} \nabla \mathbf{b}_{\omega} \text { at } A_{\beta \omega} \\
& \text { B.C.5 } \mathbf{b}_{\beta}=0 \text { at } A_{\beta \gamma} \\
& \text { B.C.6 } \mathbf{b}_{\omega}=0 \text { at } A_{\omega \gamma} \\
& \text { B.C.7(Periodicity) } \quad \mathbf{b}_{\beta}\left(\mathbf{r}+l_{i}\right)=\mathbf{b}_{\beta}(\mathbf{r}), i=1,2,3 \quad i=1,2,3 \\
& \text { B.C.8(Periodicity) } \quad \mathbf{b}_{\omega}\left(\mathbf{r}+l_{i}\right)=\mathbf{b}_{\omega}(\mathbf{r}), i=1,2,3 \quad i=1,2,3 \\
& \text { B.C.9 }\left\langle\mathbf{b}_{\beta}\right\rangle^{\beta}=0 \text { at } A_{\beta e} \\
& \text { B.C.10 }\left\langle\mathbf{b}_{\omega}\right\rangle^{\omega}=0 \text { at } A_{\omega e} \\
& \mathbf{U}_{\omega}=\frac{1}{V} \int_{A_{\omega \gamma}} \mathbf{n}_{\omega \gamma} \cdot \mathbf{D}_{\mathbf{A} \omega} \cdot \nabla \mathbf{b}_{\omega} \mathrm{d} A+\frac{1}{V} \int_{A_{\omega \beta}} \mathbf{n}_{\omega \beta} \cdot \mathbf{D}_{\mathbf{A} \omega} \cdot \nabla \mathbf{b}_{\omega} \mathrm{d} A \\
& +\frac{1}{V} \int_{A_{\omega \sigma}} \mathbf{n}_{\omega \sigma} \cdot \mathbf{D}_{\mathbf{A} \omega} \cdot \nabla \mathbf{b}_{\omega} \mathrm{d} A
\end{aligned}
$$

\section{Problem II}

$$
\begin{aligned}
\beta \text {-phase : } & \mathbf{v}_{\beta} . \nabla s_{\beta}=\nabla .\left(D_{A \beta} \nabla s_{\beta}\right)+\epsilon_{\beta}^{-1} \mathbf{S}_{\beta} \\
\text { B.C. } 1 & -\mathbf{n}_{\beta \sigma} \cdot \nabla s_{\beta}=0 \quad \text { at } A_{\beta \sigma} \\
\text { B.C. } 2 & -\mathbf{n}_{\omega \sigma} . \nabla s_{\omega}=0 \quad \text { at } A_{\omega \sigma} \\
\text { B.C. } 3 & s_{\beta}=s_{\omega} \quad \text { at } A_{\beta \omega} \\
\text { B.C. } 4 & -\mathbf{n}_{\beta \omega} \cdot D_{A \beta} \nabla s_{\beta}=-\mathbf{n}_{\beta \omega} \cdot K_{\beta \omega}^{-1} \mathbf{D}_{\mathbf{A} \omega} . \nabla s_{\omega} \quad \text { at } A_{\beta \omega} \\
\text { B.C. } 5 & s_{\beta}=1 \quad \text { at } A_{\beta \gamma}
\end{aligned}
$$




$$
\begin{aligned}
\text { B.C.6 } \quad s_{\omega}=1 \quad \text { at } A_{\omega \gamma} \\
\text { B.C.7(Periodicity) } \quad s_{\beta}\left(\mathbf{r}+l_{i}\right)=s_{\beta}(\mathbf{r}), i=1,2,3 \quad i=1,2,3 \\
\text { B.C.8(Periodicity) } \quad s_{\omega}\left(\mathbf{r}+l_{i}\right)=s_{\omega}(\mathbf{r}), i=1,2,3 \quad i=1,2,3 \\
\text { B.C.9 } \quad\left\langle s_{\beta}\right\rangle^{\beta}=0 \\
\text { B.C.10 } \quad\left\langle s_{\omega}\right\rangle^{\omega}=0 \\
\omega \text {-phase : } \quad \nabla .\left(\mathbf{D}_{\mathbf{A} \omega} \cdot \nabla s_{\omega}\right)=\epsilon_{\omega}^{-1} \mathbf{S}_{\omega} \\
\mathbf{S}_{\beta}=\frac{1}{V} \int_{A_{\beta \gamma}} \mathbf{n}_{\beta \gamma} \cdot D_{A \beta} \nabla s_{\beta} \mathrm{d} A+\frac{1}{V} \int_{A_{\beta \omega}} \mathbf{n}_{\beta \omega} \cdot D_{A \beta} \nabla s_{\beta} \mathrm{d} A \\
\quad+\frac{1}{V} \int_{A_{\beta \sigma}} \mathbf{n}_{\beta \sigma} \cdot D_{A \beta} \nabla s_{\beta} \mathrm{d} A \\
\mathbf{S}_{\omega}=\frac{1}{V} \int_{A_{\omega \gamma}} n_{\omega \gamma} \cdot \mathbf{D}_{\mathbf{A} \omega} \cdot \nabla s_{\omega} \mathrm{d} A+\frac{1}{V} \int_{A_{\omega \beta}} \mathbf{n}_{\omega \beta} \cdot \mathbf{D}_{\mathbf{A} \omega} \cdot \nabla s_{\omega} \mathrm{d} A \\
\quad+\frac{1}{V} \int_{A_{\omega \sigma}} \mathbf{n}_{\omega \sigma} \cdot \mathbf{D}_{\mathbf{A} \omega} \cdot \nabla s_{\omega} \mathrm{d} A
\end{aligned}
$$

\section{References}

Ahmadi, A., Aigueperse, A., Quintard, M.: Calculation of the effective properties describing active dispersion in porous media. Adv. Water Ressour. 24, 423-431 (2001)

Armstrong, R.T., Wildenschild, D.: Investigating the pore-scale mechanisms of microbial enhanced oil recovery. J. Petrol. Sci. Eng. 94-95, 155-164 (2012)

Bahar, T., Golfier, F., Oltéan, C.: Impact of microbial phase on NAPL dissolution in porous media. 7th International Conference on Porous Media and Annual Meeting (INTERPORE 2015), Padova, Italy, 18-21 May (2015)

Becker, J.G., Seagren, E.A.: Modeling the effects of microbial competition and hydrodynamics on the dissolution and detoxification of dense nonaqueous phase liquid contaminants. Environ. Sci. Technol. 43, 870-877 (2009)

Benioug, M., Golfier, F., Tinet, A.J., Buès, M.A., Oltean, C.: Numerical efficiency assessment of IB-LB method for 3D pore-scale modeling of flow and transport. Transp. Porous Media. 109, 1-23 (2015)

Bradford, S.A., Phelan, T.J., Abriola, L.M.: Dissolution of residual tetrachloroethylene in fractional wettability porous media. J. Contam. Hydrol. 45, 35-61 (2000)

Brun, A., Engesgaard, P.: Modelling of transport and biogeochemical processes in pollution plumes literature review and model development. J. Hydrol. 256, 1149-1159 (2002)

Brun, M., Hatfield, K.: Dimensionless parameters to summarize the influence of microbial growth and inhibition on the bioremediation of groundwater contaminants. Bioremediation 22, 877-896 (2011)

Carbonell, R.G., Whitaker, S.: Dispersion in pulsed systems. II. Theoretical developments for passive dispersion in porous media. Chem. Eng. Sci. 38, 1795-1802 (1983)

Chambon, J.C., Broholm, M.M., Binning, P.J., Bjerg, P.L.: Modeling multi-component transport and enhanced anaerobic dechlorination processes in a single fracture-clay matrix system. J. Contam. Hydrol. 112, 77-90 (2010)

Chen, M., Abriola, L.M., Amos, B.K., Suchomel, E.J., Pennell, K.D., Löffler, F.E., Christ, J.A.: Microbially enhanced dissolution and reductive dechlorination of PCE by a mixed culture. J. Contam. Hydrol. 151, 117-130 (2013)

Christ, J.A., Abriola, L.M.: Modeling metabolic reductive dechlorination in dense non-aqueous phase liquid source zones. Adv. Water Resour. 30, 1547-1561 (2006)

Chu, M., Kitanidis, P.K., McCarty, P.L.: Dependence of lumped mass transfer coefficient on scale and reactions kinetics for biologically enhanced NAPL dissolution. Adv. Water Resour. 30, 1618-1629 (2007)

Cirpka, O.A., Frind, E.O., Helmig, R.: Numerical simulation of biodegradation controlled by transverse mixing. J. Contam. Hydrol. 40, 159-82 (1999)

Clement, T.P., Gautam, T.R., Lee, K.K., Truex, M.J., Davis, G.B.: Modeling of DNAPL-dissolution, ratelimited sorption and biodegradation reactions in groundwater systems. Bioremed. J. 8, 47-64 (2004) 
Corapcioglu, M.Y., Yoon, S., Chowdhury, S.: Pore-scale analysis of NAPL blob dissolution and mobilization in porous media. Transp. Porous Media. 79, 419-442 (2009)

Davit, Y., Debenest, G., Wood, B.D., Quintard, M.: Modeling non-equilibrium mass transport in biologically reactive porous media. Adv. Water Ressour. 33, 1075-1093 (2010)

Davit, Y., Iltis, G., Debenest, G., Veran-Tissoires, S., Wildenschild, D., Gerino, M., Quintard, M.: Imaging biofilm in porous media using X-ray computed microtomography. J. Microsc. 242, 15-25 (2010)

Gaganis, P., Karapanagioti, H.K., Burganos, V.N.: Modeling multicomponent NAPL transport in the unsaturated zone with the constituent averaging technique. Adv. Water Resour. 25, 723-732 (2002)

Garcia-Junco, M., Gomez-Lahoz, C., Niqui-Arroyo, J.-L., Ortega-Calvo, J.L.: Biosurfactant- and biodegradation-enhanced partitioning of polycyclic aromatic hydrocarbons from nonaqueous-phase liquids. Environ. Sci. Technol. 37, 2988-2996 (2003)

Golfier, F., Wood, B.D., Orgogozo, L., Quintard, M., Buès, M.A.: Biofilms in porous media: development of macroscopic transport equations via volume averaging with closure for local mass equilibrium conditions. Adv. Water Ressour. 32, 463-485 (2009)

Gray, W.G., Leijnse, A., Kolar, R.L, Blain, C.A.: Mathematical Tools for Changing Spatial Scales in the Analysis of Physical Systems, pp. 2985-2996. CRC Press, Boca Raton (1993)

Hunt, J.R., Sitar, N.: Nonaqueous phase liquid transport and cleanup 1. Analysis of mechanisms. Water Ressour. Res. 24, 1247-1258 (1988)

Kindred, J.S., Celia, M.A.: Contaminant transport and biodegradation 2. Conceptual model and test simulations. Water Resour. Res. 25, 1149-1159 (1989)

Lugo-Mèndez, H.D., Valdès-Parada, F.J., Porter, M.L., Wood, B.D., Ochoa-Tapia, J.A.: Upscaling diffusion and nonlinear reactive mass transport in homogeneous porous media. Transp. Porous Media. 107, 683716 (2015)

Mayer, A.S., Miller, C.T.: An experimental investigation of pore-scale distributions of Nonaqueous phase liquids at residual saturation. Transp. Porous Media. 10, 57-80 (1993)

Miller, C.T., Poirier-Mcnell, M.M.: Dissolution of trapped nonaqueous phase liquids. Water Resour. Res. 26, 2783-2796 (1990)

Orgogozo, L., Golfier, F., Buès, M.A., Quintard, M., Kone, T.: A dual-porosity theory for solute transport in biofilm-coated porous media. Adv. Water Ressour. 62, 266-279 (2013)

Osswald, P., Baveye, P., Block, J.C.: Bacterial influence on partitioning rate during the biodegradation of styrene in a biphasic aqueous-organic system. Biodegradation 7, 297-302 (1996)

Paulsen, J.E., Ekrann, S., Oppen, E.: Visualisation of bacterial degradation and mobilisation of oil in a porous medium. Environ. Geol. 38, 1075-1093 (1999)

Picioreanu, C., van Loosdrecht, M.M., Heijnen, J.J.: Effect of Diffusive and Convective Substrate Transport on Biofilm Structure Formation: A Two-Dimensional Modeling Study, Biotechnology and Bioengineering, p. 69 (2000)

Quintard, M., Whitaker, S.: One and two equation models for transient diffusion processes in two-phase systems. Adv. Heat Transf. 23, 369-464 (1993)

Quintard, M., Whitaker, S.: Convection, dispersion, and interfacial transport of contaminants. Adv. Water Ressour. 17, 221-239 (1994)

Quintard, M., Whitaker, S.: Transport in ordered and disordered porous media. II. Generalized volume averaging. Transp. Porous Media. 14, 179-206 (1994)

Quintard, M., Whitaker, S.: Dissolution of an immobile phase during flow in porous media. Ind. Eng. Chem. Res 38, 833-844 (1999)

Ray, S., Peters, C.A.: Changes in microbiological metabolism under chemical stress. Chemosphere 71, 474483 (2008)

Saravanan, P., Pakshirajan, K., Saha, P.: Growth kinetics of an indigenous mixed microbial consortium during phenol degradation in a batch reactor. Bioresour. Technol. 79, 205-209 (2008)

Schenk, O., Gärtner, K.: Solving unsymmetric sparse systems of linear equations with PARDISO. J. Future Gener. Comput. Syst. 20, 475-487 (2004)

Schubert, M., Paschke, A., Lau, S., Geyer, W., Knöller, K.: Radon as a naturally occurring tracer for the assessment of residual NAPL contamination of aquifers. Environ. Pollut. 145, 920-927 (2007)

Semprini, L., McCarty, P.L.: Comparison between model simulations and field results for in situ biorestoration of chlorinated aliphatics.Part 1. Ground Water 29, 365-74 (1991)

Seyedabbasi, M.A., Farthing, M.W., Imhoff, P.T.: The influence of wettability on NAPL dissolution fingering. Adv. Water Resour. 31, 1687-1696 (2008)

Seymour, J.D., Codd, S.L., Gjersing, E.L., Stewart, P.S.: Magnetic resonance microscopy of biofilm structure and impact on transport in a capillary bioreactor. J. Magn. Reson. 167, 322-327 (2004)

Singh, R., Olson, M.S.: Kinetics of trichloroethylene and toluene toxicity to Pseudomonas Putida F1. Environ. Toxicol. Chem. 29, 56-63 (2009) 
Sivasankar, P., Suresh, G.: Numerical modelling of enhanced oil recovery by microbial flooding under nonisothermal conditions. J. Petrol. Sci. Eng. 124, 161-172 (2014)

Soudmand-asli, A., Ayatollahi, S.S., Mohabatkar, H., Zareie, M., Shariatpanahi, S.: The in situ microbial enhanced oil recovery in fractured porous media. J. Petrol. Sci. Eng. 58, 161-172 (2007)

Soulaine, C., Debenest, G., Quintard, M.: Upscaling multi-component two-phase flow in porous media with partitioning coefficient. Chem. Eng. Sci. 66, 6180-6192 (2011)

Valdès-Parada, F.J., Porter, M.L., Narayanaswamy, K., Ford, R.M., Wood, B.D.: Upscalling microbial chemotaxis in porous media. Adv. Water Resour. 32, 1413-1428 (2009)

Whitaker, S.: The Method of Volume Averaging. Kluwer Academic Publishers, Dordrecht (1999)

Wood, B.D., Golfier, F., Quintard, M.: Dispersive transport in porous media with biofilms: local mass equilibrium in simple unit cells. Int. J. Environ. Waste Manage. 7, 24-48 (2011)

Wood, B.D., Whitaker, S.: Diffusion and reaction in biofilms. Chem. Eng. Sci. 53, 397-425 (1998)

Wood, B.D., Whitaker, S.: Multi-species diffusion and reaction in biofilms and cellular media. Chem. Eng. Sci. 55, 3397-3418 (2000)

Yang, Y.R., McCarty, P.L.: Biologically enhanced dissolution of tetrachloroethene DNAPL. Environ. Sci. Technol. 34, 2979-2984 (2000) 